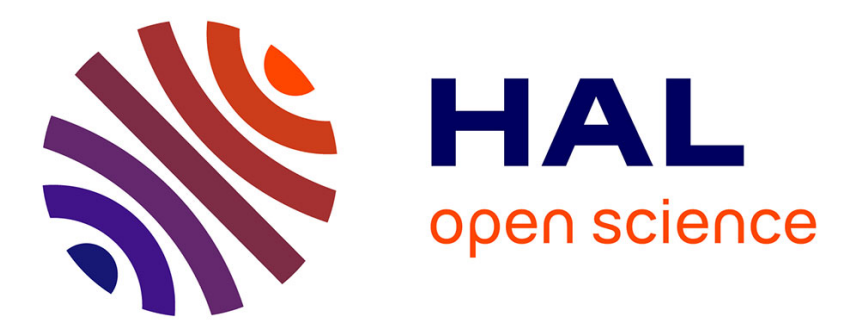

\title{
Normal-mode theory of a rotating Earth model using a Lagrangian perturbation of a spherical model of reference
}

Yves Rogister, Rochester M.G.

\section{- To cite this version:}

Yves Rogister, Rochester M.G.. Normal-mode theory of a rotating Earth model using a Lagrangian perturbation of a spherical model of reference. Geophysical Journal International, 2004, 159, pp.874908. 10.1111/j.1365-246x.2004.02447.x . insu-00675758

\section{HAL Id: insu-00675758}

https://hal-insu.archives-ouvertes.fr/insu-00675758

Submitted on 12 Mar 2021

HAL is a multi-disciplinary open access archive for the deposit and dissemination of scientific research documents, whether they are published or not. The documents may come from teaching and research institutions in France or abroad, or from public or private research centers.
L'archive ouverte pluridisciplinaire HAL, est destinée au dépôt et à la diffusion de documents scientifiques de niveau recherche, publiés ou non, émanant des établissements d'enseignement et de recherche français ou étrangers, des laboratoires publics ou privés. 


\title{
Normal-mode theory of a rotating earth model using a Lagrangian perturbation of a spherical model of reference
}

\author{
Yves Rogister $^{1}$ and Michael G. Rochester ${ }^{2}$ \\ ${ }^{1}$ Ecole et Observatoire des Sciences de la Terre, 5, rue René Descartes, F-67084, Strasbourg Cedex, France. E-mail: Yves.Rogister@eost-u.strasbg.fr \\ ${ }^{2}$ Department of Earth Sciences, Memorial University of Newfoundland, St. John's, Newfoundland, A1B 3X5, Canada
}

Accepted 2004 August 9. Received 2004 August 3; in original form 2003 August 13

\begin{abstract}
SUMMAR Y
The normal-mode theory of a rotating earth model is based on the superposition of two perturbations. The first one is the perturbation of a spherically averaged model of reference by rotation: it provides the rotating earth model. The second one is a perturbation of the rotating model: it is a normal mode. In both cases, we consider Lagrangian perturbations. This implies that we define first a new coordinate system in the spherical configuration of reference. These coordinates, which are non-orthogonal, are such that the parameters of the spherical model depend on one of the coordinates only. The relation between the physical spherical coordinates in the rotating configuration and the new coordinates involves the radial discrepancy $h$ between the spherical model of reference and the rotating model. We assume that, prior to being perturbed, the rotating model is in hydrostatic equilibrium. We determine the shape of the rotating configuration to the second order in $h$, using the theory of hydrostatic equilibrium figures. Next, we write the equations of motion of the rotating model in the new coordinate system. We suppose that the stress-strain relation is linearly elastic and isotropic. By inserting the analytical solution for the tilt-over mode in the equations of motion, we show that the terms containing the initial equilibrium gravity must be computed to the second order in $h$. Finally, we separate the variables in the equations of motion by expanding the unknown functions on the basis of surface spherical harmonics. We obtain an infinite set of coupled first-order ordinary differential equations which, if truncated, is suitable for numerical integration.
\end{abstract}

Key words: Earth's rotation, hydrostatic figures, normal modes, nutation.

\section{INTRODUCTION}

We can arbitrarily divide the spectrum of elastic-gravitational free oscillations of a rotating earth model into three sets. To the first infinite set belong the seismic normal modes whose periods are below one hour and the three translational modes of oscillation of the inner core, usually named the Slichter modes, whose periods are a few hours for most realistic earth models. The seismic free oscillations also exist for non-rotating, spherical earth models. Their eigenfrequencies and eigenfunctions are little changed by rotation and the non-spherical shape of the rotating body. Hundreds of seismic modes have been observed. The inertia-gravity modes of the liquid outer core form the second infinite set of free oscillations. Their computed eigenperiods are above a few hours. Rotation strongly affects the core modes of a non-rotating, spherical model. Core modes are still unobserved. The last set includes the five principal rotational modes: the tilt-over mode (TOM), the free core nutation (FCN), the free inner core nutation (FICN), the Chandler wobble (CW) and the inner core wobble (ICW) (Mathews et al. 1991a,b). Except for the TOM, the eigenperiods of the rotational modes heavily depend on the shape of the rotating body. The TOM period is diurnal, the FCN and FICN periods are nearly diurnal, the CW period is about 430 sidereal days, and the computed period of the ICW is of the order of a few years for realistic earth models. The main feature of the eigenfunctions of the rotational modes is a dominant rigid rotation of the mantle (CW), of the inner core (FICN, ICW), of the outer core (FCN), or of the whole earth (TOM) about an equatorial axis. The CW, the FCN, and the FICN have been observed, whereas the ICW is still unobserved. The TOM is presented in more detail in Section 6 of this paper.

In order to study theoretically the normal modes of a slowly and steadily rotating body it is first necessary to relate the unperturbed physical properties of this body to the physical properties of a corresponding non-rotating, spherical model of reference. This means that we must compute the perturbation of the spherical model by rotation before we consider the free oscillations of the rotating body. The usual two descriptions of a perturbation are provided by the Lagrangian and the Eulerian approaches. The Lagrangian description of a perturbation of a quantity consists in evaluating the variation of that quantity between the initial position of a material particle and its current position. The Eulerian description consists in evaluating its variation in time at a fixed position in space. In Smith's (1974) theory of the infinitesimal 
elastic-gravitational motions of a rotating earth model, the physical quantities describing the rotating model, i.e. the density and the Lamé parameters, are related to those of the mean spherical model of reference by a first-order Eulerian perturbation scheme. The rotating model being assumed to be initially in hydrostatic equilibrium in a co-rotating frame of reference, Clairaut's theory of hydrostatic figures of equilibrium (Zharkov \& Trubitsyn 1978, pp. 221-295; Denis 1989; Denis et al. 1998) shows that, in a first-order approximation, the shape of the rotating body is a slightly flattened ellipsoid of revolution whose minor axis is parallel to the axis of rotation. Moreover, the density of the mean spherical model being known as a function of the radius, Clairaut's theory provides the small radial distance $h$ between a mean spherical surface where the density takes on a given value and the corresponding rotating ellipsoidal surface where the density takes on the same value. This radial distance is a function of the radius $q$ of the spherical surface and of the colatitude $\theta$, which we will denote by $\chi$ throughout the paper, and depends linearly on the flattening of the ellipsoidal surface. The flattening is here defined as $(a-c) / a$, where $a$ and $c$ are the equatorial and polar radii, respectively. In a Eulerian perturbation scheme, the density at a geometrical point of the rotating ellipsoidal model is given in terms of the density of the mean spherical model at the same geometrical point. Since the density at a point of the rotating ellipsoidal model is equal to the density of the mean spherical model at the radial distance $h$, a first-order Taylor's expansion furnishes the density of the rotating model in terms of the density of the mean model at the same geometrical point. Smith (1974) also supposes that the surfaces of constant Lamé parameters coincide with the surfaces of constant density. Consequently, the Lamé parameters of the rotating model are also determined when the equation of the isopycnic surfaces is known. Once the physical parameters of the rotating model are known, the Lagrangian vector equation of motion of the rotating earth and the associated Poisson's equation for the variation of the gravitational potential are transformed into scalar ordinary differential equations by using spherical coordinates and spherical harmonics.

Although Smith's pioneering work and the numerical results that he subsequently published $(1976,1977)$ are mostly consistent, we can point out three improvements that either have been made or can be made to a normal-mode theory of a rotating earth.

First, owing to Taylor's expansion of the parameters of the spherical model, their derivatives are introduced into the theory. This may be harmful to the numerical results derived from the theory because the derivatives of the physical parameters of published spherical models of reference may not be well constrained by observations (e.g. Dziewonski et al. 1975; Dziewonski \& Anderson 1981). Moreover, when the parameters of the reference model are given at discrete radius values instead of as piecewise continuous functions of the radius, the differential scalar equations that contain their radial derivatives are not suitable for numerical integration. In this respect, it is noteworthy that the transformation of the vector equation of motion of a non-rotating, spherical model into a set of scalar differential equations was designed to avoid the use of the radial derivatives of the density and of the Lamé parameters (Alterman et al. 1959).

Second, in small regions near the boundaries where the density or the Lamé parameters are discontinuous, Taylor's expansion of them fails. Indeed, rotation has deformed the spherical model of reference into an ellipsoidal model, and the material particles have been displaced along a radial distance $h(q, \chi)$ from their initial position. The boundaries have moved and have occupied successively geometrical points where, consequently, the physical parameters have changed abruptly during the deformation. This prevents Taylor's expansion at these geometrical points as well as the imposition of the continuity conditions, at the ellipsoidal boundaries, to the infinitesimal deformation of the ellipsoidal rotating model. When Smith imposes the continuity conditions at the ellipsoidal boundaries, he must resort to a Lagrangian perturbation of the spherical configuration by rotation. Actually, he imposes modified continuity conditions at the spherical boundaries of the reference model. The perturbation of the spherical model by rotation is, therefore, treated from a Eulerian point of view in the equations of motion and from a Lagrangian point of view in the boundary conditions.

Third, it has been shown independently by Schastok (1997) and Rogister (2001) that the proper theoretical treatment of the rotational motions of a rotating, slightly flattened hydrostatic earth model requires that terms of second order in the flattening be included in the equations governing the infinitesimal deformations of the rotating model. Both studies are based on the theory developed by Smith, who neglects the terms of orders higher than the first in the flattening. The arguments invoked by Schastok and by Rogister are different: Schastok shows that the solution for the rotational motions of a particular model, namely an incompressible and homogeneous solid ellipsoid, is correct only if second-order terms in the flattening are taken into account, whereas Rogister shows that the solution for one particular rotational eigenmode, that is the TOM, satisfies the equations of motion only if second-order terms are included in them. The TOM is independent of earth model and its frequency is exactly one sidereal day. Nevertheless, neither Schastok nor Rogister retains all the terms of second order in the flattening.

A further difficulty encountered in Smith's theory has to do with the representation of the infinitesimal deformation of the rotating model as a series of coupled terms. Indeed, the infinitesimal displacement vector is split into a spheroidal part and a toroidal part, and each part is expanded on the basis of vector spherical harmonics. In the non-rotating spherical case, the spheroidal components do not couple with the toroidal components, none of them depends on the harmonic order $m$, and components of different harmonic degrees do not couple with each other. This implies that a harmonic deformation of degree $\ell$ and order $m$ does not couple to any other harmonic deformation of degree $\ell^{\prime} \neq \ell$ or of order $m \prime \neq m$, and that it is a solution of a differential system of finite order. In the rotating case, the spheroidal and toroidal harmonic components depend on $m$, but components of different azimuthal order still do not couple. A spheroidal (toroidal) component of harmonic degree $\ell$ couples with the spheroidal (toroidal) components of degrees $\ell-2, \ell+2, \ell-4, \ell+4, \ldots$ and with the toroidal (spheroidal) components of degrees $\ell-1, \ell+1, \ell-3, \ell+3, \ldots$ Thus, the displacement vector is expressed as a series that must be truncated arbitrarily at some finite level for the purpose of numerical resolution. The replacement of a series by a sum is sometimes questionable, in particular when dealing with the inertia-gravity modes of the liquid core.

The aim of this paper is to build a normal-mode theory of a rotating model that is based both on a Lagrangian perturbation of the spherical model of reference by rotation and on a Lagrangian infinitesimal perturbation of the rotating configuration (Fig. 1). 


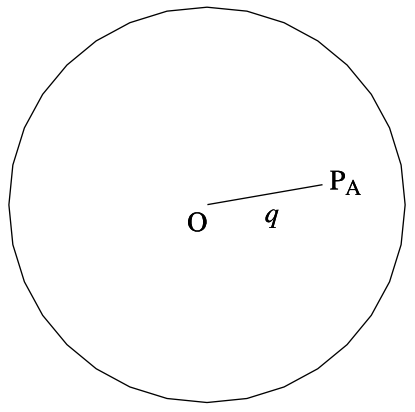

A. Non-rotating spherical reference model
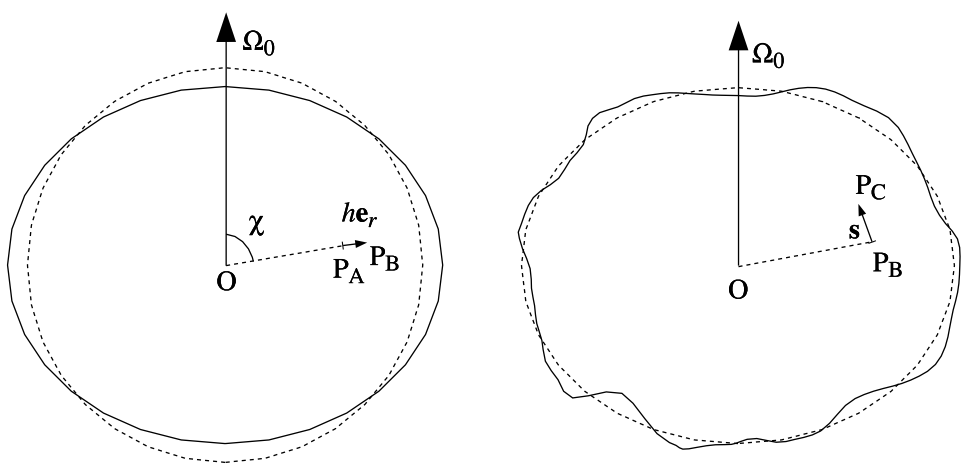

B. Steadily-rotating spheroidal model
C. Spheroidal model perturbed by earthquake, tide...

Figure 1. Meridional cross-section of a spherical reference earth model (A), the corresponding rotating spheroidal model (B), and the perturbed rotating spheroidal model (C). Radial Lagrangian displacement $h(q, \chi) \mathbf{e}_{r}$, where $\mathbf{e}_{r}$ is the radial unit vector, carries a mass element from $\mathrm{P}_{\mathrm{A}}(\mathrm{q}, \chi, v)$ to $\mathrm{P}_{\mathrm{B}}(\mathrm{q}+\mathrm{h}, \chi, v)$ and Lagrangian displacement $\mathbf{s}$ carries a mass element from $\mathrm{P}_{\mathrm{B}}$ to $\mathrm{P}_{\mathrm{C}}$. The first perturbation, by steady rotation $\boldsymbol{\Omega}_{0}$, maintains hydrostatic equilibrium. The second infinitesimal perturbation may be a tidal deformation, a normal-mode vibration, a nutational oscillation, ect.

Therefore, a physical parameter at a point in the ellipsoidal configuration is equal to the same parameter evaluated at the corresponding point in the spherical configuration. There is no need to expand the parameters in Taylor's series.

Moreover, both in the equations of motion of the rotating model and in the boundary conditions that the infinitesimal motion must satisfy, we treat the perturbation of the spherical model of reference from a Lagrangian point of view.

Practically, adopting a Lagrangian point of view consists in defining a new set of non-orthogonal coordinates $(q, \chi, \nu)$ in the spherical configuration of reference, such that the parameters of the spherical model of reference are constant over the surfaces $q=$ constant. The coordinate $q$ is related to the radial coordinate $r$ of the physical spherical coordinates in the rotating configuration by $r=q+h$, where, as we have seen, $h$ is given by the theory of hydrostatic figures of equilibrium. The angular coordinates $\chi$ and $v$ correspond to the colatitude $\theta$ and longitude $\varphi$, respectively. A similar change of coordinates was suggested by Jeffreys (1942) in order to solve the tidal equations.

To determine to what order in $h$ the equations of motion must be computed, we search for the conditions for the TOM to be a solution of the equations of motion. We then show that certain terms must be computed up to the second order in $h$. It is therefore necessary to compute the hydrostatic shape of the rotating body to second order. To this order of approximation, unless the body is homogeneous, the hydrostatic figure of the body is no longer an ellipsoid of revolution but, of course, remains close to it.

It is well known that the representation of an infinitesimal displacement by a series of coupled spheroidal and toroidal vectors cannot be avoided as long as spherical harmonics are used to describe the deformation. The reason is that the symmetry properties of the rotating body are not the same as those of the spherical harmonics. If the rotating body is homogeneous, its hydrostatic shape is, rigorously, an ellipsoid of revolution, and the flattening of the equisurfaces is a constant. In this case, using spheroidal coordinates and spheroidal harmonics to represent the deformation may lead to the decoupling of the deformations of different harmonic degrees and of different harmonic orders (Bryan 1889; Usami 1962). If the rotating body is not homogeneous, there are no basis functions adapted to the symmetry of the body. It is therefore customary to use the surface spherical harmonics. We will do so in this paper, and it should not be expected that the coupling chain that represents the displacement vector will be simpler than the one obtained in the theory of Smith (1974).

The paper is organized as follows. In Section 2, we define the new set of coordinates $(q, \chi, v)$ in the spherical configuration of reference. In Section 3, we establish useful formulae of tensor calculus in the coordinate system $(q, \chi, v)$. We also introduce the usual decomposition of a vector into spheroidal and toroidal vector fields. Section 4 is devoted to the hydrostatic figures of equilibrium. The Lagrangian perturbation of a spherical model of reference by rotation is the first perturbation that we must take into account. We suppose that, on very long timescales, the Earth behaves like a liquid body. Therefore, the theory of hydrostatic figures of equilibrium is adequate for determining the perturbation by rotation. The goal of this theory is to compute the radial discrepancy $h$ between the spherical model of reference and the steadily rotating hydrostatic configuration. In Sections 5 to 7, we consider an infinitesimal perturbation of the rotating model. Our aim is to transform the partial differential equations of motion of the rotating earth into ordinary differential equations over radius $q$ by separating the variables $q, \chi$ and $\nu$. The vector equations of motion and the associated boundary conditions are given in Section 5. We assume that the stress-strain relation is linearly elastic and isotropic. Among the possible motions, only the normal modes are considered. To separate the variables $q, \chi$, and $v$ in the equations of motion, it is necessary to use an approximate expression for $h$. Thus, in Section 6, we show how the TOM imposes constraints on the approximations allowed for $h$ and we discuss the validity of our conclusions for the other rotational motions. In Section 7, we expand the Fourier-transformed equations of motion on the basis of the surface spherical harmonics, allowing for the separation of the variables $q$, $\chi$, and $v$. Therefore, we obtain the first-order ordinary differential equations governing the harmonic components of the displacement vector, the traction and the gravitational field variation of a rotating earth model. 


\section{COORDINATE SYSTEM IN THE SPHERICAL CONFIGURATION OF REFERENCE}

In this section, we define a set of non-orthogonal curvilinear coordinates $(q, \chi, v)$ such that the parameters of the reference earth model depend on $q$ only. We introduce the natural basis associated with the coordinates $(q, \chi, v)$, the components of the metric tensor and the Christoffel symbols. The reader is referred to, for example, Synge \& Schild (1978) for an introduction to Riemannian space, of which the space of the reference configuration is a particular case.

\subsection{Definition of the coordinate system and basis vectors}

We consider a spherically symmetric model of reference given by the density $\rho_{0}$ and the Lamé parameters $\lambda_{0}$ and $\mu_{0}$ as functions of the radius $q$. We then consider a perturbation that brings a material particle from the position $q \mathbf{e}_{r}$ to the position $r \mathbf{e}_{r}$, where $\mathbf{e}_{r}$ is the radial unit vector and where $r$ and $q$ are related by

$r=q+h(q, \chi)$

$\theta=\chi$

$\varphi=v$.

$h$ is, for the time being, an arbitrary twice continuously differentiable function of $q$ and $\chi$. The coordinates $r, \theta$ and $\varphi$ are the usual spherical coordinates, whereas $q, \chi$ and $v$ form a new set of coordinates. Because we will consider only an axially symmetric perturbation of the reference configuration, $h$ does not depend on $v$. The covariant basis vectors associated with the coordinate system $q, \chi, v$ are

$\mathbf{e}_{q}=\left(1+\frac{\partial h}{\partial q}\right) \mathbf{e}_{r}$

$\mathbf{e}_{\chi}=\frac{\partial h}{\partial \chi} \mathbf{e}_{r}+(q+h) \mathbf{e}_{\theta}$,

$\mathbf{e}_{v}=(q+h) \sin \chi \mathbf{e}_{\varphi}$,

where $\mathbf{e}_{\theta}$ and $\mathbf{e}_{\varphi}$ are, along with $\mathbf{e}_{r}$, the unit vectors of the local basis associated with the spherical coordinates $(r, \theta, \varphi)$. The basis vectors $\mathbf{e}_{q}, \mathbf{e}_{\chi}$ and $\mathbf{e}_{v}$ are not normalized, and the vectors $\mathbf{e}_{q}$ and $\mathbf{e}_{\chi}$ are not mutually orthogonal. The vectors $\mathbf{e}_{q}, \mathbf{e}_{\chi}, \mathbf{e}_{v}$ form the natural basis of the coordinate system $q, \chi, v$. The position vector $\mathbf{r}=r \mathbf{e}_{r}$ then becomes

$\mathbf{r}=\frac{q+h}{1+\frac{\partial h}{\partial q}} \mathbf{e}_{q}$.

The unit normal to a surface $q=$ constant is given by

$\mathbf{n}=\frac{\frac{\partial \mathbf{r}}{\partial \chi} \times \frac{\partial \mathbf{r}}{\partial \nu}}{\left|\frac{\partial \mathbf{r}}{\partial \chi} \times \frac{\partial \mathbf{r}}{\partial \nu}\right|}=\frac{1}{(q+h) \sqrt{(q+h)^{2}+\left(\frac{\partial h}{\partial \chi}\right)^{2}}}\left[\frac{(q+h)^{2}+\left(\frac{\partial h}{\partial \chi}\right)^{2}}{1+\frac{\partial h}{\partial q}} \mathbf{e}_{q}-\frac{\partial h}{\partial \chi} \mathbf{e}_{\chi}\right]$.

From now on, we will consider the curvilinear coordinates $(q, \chi, v)$ only.

\subsection{Metric tensor}

The covariant components of the symmetric metric tensor $\boldsymbol{g}$ are defined by

$g_{i j}=\mathbf{e}_{i} \cdot \mathbf{e}_{j}$,

where the subscripts $i$ and $j$ designate $q, \chi$ or $\nu$. The non-vanishing covariant components of the metric tensor are

$g_{q q}=\left(1+\frac{\partial h}{\partial q}\right)^{2}$

$g_{q \chi}=\left(1+\frac{\partial h}{\partial q}\right) \frac{\partial h}{\partial \chi}$

$g_{\chi \chi}=\left(\frac{\partial h}{\partial \chi}\right)^{2}+(q+h)^{2}$

$g_{v v}=(q+h)^{2} \sin ^{2} \chi$. 
Inverting, we obtain the non-vanishing contravariant components of the metric tensor:

$g^{q q}=\frac{\left(\frac{\partial h}{\partial \chi}\right)^{2}+(q+h)^{2}}{\left(1+\frac{\partial h}{\partial q}\right)^{2}(q+h)^{2}}$

$g^{q \chi}=-\frac{\frac{\partial h}{\partial \chi}}{\left(1+\frac{\partial h}{\partial q}\right)(q+h)^{2}}$,

$g^{\chi x}=\frac{1}{(q+h)^{2}}$,

$g^{\nu v}=\frac{1}{(q+h)^{2} \sin ^{2} \chi}$.

The determinant $g$ of the metric tensor is

$g=(q+h)^{4}\left(1+\frac{\partial h}{\partial q}\right)^{2} \sin ^{2} \chi$

\subsection{Christoffel symbols}

The Christoffel symbols of the second kind are defined by

$\Gamma_{j k}^{i}=\frac{1}{2} g^{i l}\left(g_{l j, k}+g_{l k, j}-g_{j k, l}\right)$,

where the Einstein summation convention is used and the comma in a subscript position designates a partial derivative with respect to $q$, $\chi$ or $v$. Noting that $\Gamma_{j \mathrm{k}}^{i}=\Gamma_{k \mathrm{j}}^{i}$, some algebra gives the non-vanishing Christoffel symbols:

$\Gamma_{q q}^{q}=\frac{1}{1+\frac{\partial h}{\partial q}} \frac{\partial^{2} h}{\partial q^{2}}$,

$\Gamma_{q \chi}^{q}=\frac{1}{1+\frac{\partial h}{\partial q}} \frac{\partial^{2} h}{\partial q \partial \chi}-\frac{1}{q+h} \frac{\partial h}{\partial \chi}$,

$\Gamma_{\chi \chi}^{q}=\frac{1}{\left(1+\frac{\partial h}{\partial q}\right)(q+h)}\left[-(q+h)^{2}+(q+h) \frac{\partial^{2} h}{\partial \chi^{2}}-2\left(\frac{\partial h}{\partial \chi}\right)^{2}\right]$,

$\Gamma_{v v}^{q}=\frac{\sin \chi}{1+\frac{\partial h}{\partial q}}\left[\frac{\partial h}{\partial \chi} \cos \chi-(q+h) \sin \chi\right]$,

$\Gamma_{\chi \chi}^{\chi}=\frac{2}{q+h} \frac{\partial h}{\partial \chi}$

$\Gamma_{q \chi}^{\chi}=\frac{1+\frac{\partial h}{\partial q}}{q+h}$,

$\Gamma_{q v}^{v}=\Gamma_{q \chi}^{\chi}$

$\Gamma_{\chi \nu}^{\nu}=\frac{1}{(q+h)} \frac{\partial h}{\partial \chi}+\frac{\cos \chi}{\sin \chi}$

$\Gamma_{v v}^{\chi}=-\sin \chi \cos \chi$

The metric tensor and the Christoffel symbols are the quantities that we need to introduce tensor calculus in a Riemannian space. We turn to tensor calculus in the coordinate system $q, \chi, v$ in the next section. 


\section{TENSOR ANALYSIS IN THE SPHERICAL CONFIGURATION OF REFERENCE}

We now collect a series of useful formulae of tensor calculus in coordinates $(q, \chi, v)$. These formulae include: the components of the gradient of a scalar function, the divergence of a vector field, the Laplacian of a scalar function, the Helmholtz representation of a vector field that allows for the splitting of that vector field into a spheroidal vector and a toroidal vector, the components of the small strain tensor, and the divergence of a tensor field. Again, the reader is referred to, for example, Synge \& Schild (1978) for basic definitions of tensor calculus.

\subsection{Gradient of a scalar function}

The covariant components of the gradient of a scalar function $f(q, \chi, v)$ are defined by

$(\nabla f)_{i}=f_{, i}$.

Its contravariant components are obtained by index-raising:

$(\nabla f)^{i}=g^{i j} f_{, j}$,

i.e.

$(\nabla f)^{q}=\frac{\left(\frac{\partial h}{\partial \chi}\right)^{2}+(q+h)^{2}}{\left(1+\frac{\partial h}{\partial q}\right)^{2}(q+h)^{2}} \frac{\partial f}{\partial q}-\frac{\frac{\partial h}{\partial \chi}}{\left(1+\frac{\partial h}{\partial q}\right)(q+h)^{2}} \frac{\partial f}{\partial \chi}$,

$(\nabla f)^{\chi}=-\frac{\frac{\partial h}{\partial \chi}}{\left(1+\frac{\partial h}{\partial q}\right)(q+h)^{2}} \frac{\partial f}{\partial q}+\frac{1}{(q+h)^{2}} \frac{\partial f}{\partial \chi}$,

$(\nabla f)^{v}=\frac{1}{(q+h)^{2} \sin ^{2} \chi} \frac{\partial f}{\partial v}$.

\subsection{Divergence of a vector field}

If $\mathbf{v}=v^{q} \mathbf{e}_{q}+v^{\chi} \mathbf{e}_{\chi}+v^{v} \mathbf{e}_{v}$ is a differentiable vector function of $q, \chi$, and $v$, its divergence is the scalar

$\boldsymbol{\nabla} \cdot \mathbf{v}=v_{; i}^{i}=v_{, i}^{i}+\Gamma_{j i}^{i} v^{j}$,

where the semicolon in a subscript position denotes the covariant derivative. We find that

$\boldsymbol{\nabla} \cdot \mathbf{v}=\frac{\partial v^{q}}{\partial q}+\frac{\partial v^{\chi}}{\partial \chi}+\frac{\partial v^{v}}{\partial v}+\left(\frac{1}{1+\frac{\partial h}{\partial q}} \frac{\partial^{2} h}{\partial q^{2}}+2 \frac{1+\frac{\partial h}{\partial q}}{q+h}\right) v^{q}+\left(\frac{1}{1+\frac{\partial h}{\partial q}} \frac{\partial^{2} h}{\partial q \partial \chi}+\frac{2}{q+h} \frac{\partial h}{\partial \chi}+\frac{\cos \chi}{\sin \chi}\right) v^{\chi}$.

\subsection{Laplacian of a scalar function}

If $f$ is a twice differentiable function of $q, \chi$ and $v$, the Laplacian of $f$ is the scalar

$\nabla^{2} f=\nabla \cdot \nabla f=\left(g^{i j} f_{, j}\right)_{; i}$,

from which we deduce that

$$
\begin{aligned}
(q+h)^{2} \nabla^{2} f= & \frac{1}{\left(1+\frac{\partial h}{\partial q}\right)^{2}}\left[\left(\frac{\partial h}{\partial \chi}\right)^{2}+(q+h)^{2}\right] \frac{\partial^{2} f}{\partial q^{2}}-2 \frac{\partial h}{\partial \chi} \frac{1}{\left(1+\frac{\partial h}{\partial q}\right)} \frac{\partial^{2} f}{\partial q \partial \chi}+\frac{\partial^{2} f}{\partial \chi^{2}}+\frac{1}{\sin ^{2} \chi} \frac{\partial^{2} f}{\partial \nu^{2}}+\left[\frac{\cos \chi}{\sin \chi}-\frac{2}{(q+h)} \frac{\partial h}{\partial \chi}\right] \frac{\partial f}{\partial \chi} \\
& +\frac{1}{\left(1+\frac{\partial h}{\partial q}\right)}\left\{2(q+h)-\frac{\partial^{2} h}{\partial \chi^{2}}+\frac{\partial h}{\partial \chi}\left[\frac{2}{\left(1+\frac{\partial h}{\partial q}\right)} \frac{\partial^{2} h}{\partial q \partial \chi}-\frac{\cos \chi}{\sin \chi}\right]-\left[\left(\frac{\partial h}{\partial \chi}\right)^{2}+(q+h)^{2}\right] \frac{\partial^{2} h}{\partial q^{2}} \frac{1}{\left(1+\frac{\partial h}{\partial q}\right)^{2}}\right\} \frac{\partial f}{\partial q} .
\end{aligned}
$$

\subsection{Helmholtz representation of a vector field}

In what follows, we will need the scalar product and the vector product of two vectors, so we define these now. The scalar product of two vectors was introduced when we computed the components of the metric tensor. We recall that the scalar product of two vectors $\mathbf{u}$ and $\mathbf{v}$ is the scalar defined by

$\mathbf{u} \cdot \mathbf{v}=u_{i} v^{i}=u^{i} v_{i}$. 
The vector product of $\mathbf{u}$ and $\mathbf{v}$ is the pseudo-vector $\mathbf{w}$ whose contravariant components are

$w^{q}=\frac{1}{\sqrt{g}}\left(u_{\chi} v_{v}-u_{\nu} v_{\chi}\right), w^{\chi}=\frac{1}{\sqrt{g}}\left(u_{\nu} v_{q}-u_{q} v_{v}\right), w^{\nu}=\frac{1}{\sqrt{g}}\left(u_{q} v_{\chi}-u_{\chi} v_{q}\right)$.

The Helmholtz representation of a vector field $\mathbf{s}$ is

$\mathbf{s}=U \frac{\mathbf{r}}{r}+\frac{\mathbf{r}}{r} \times[\nabla \times(V \mathbf{r})]+\nabla \times(W \mathbf{r})$,

where $U, V$ and $W$ are three scalar functions. The first two terms on the right-hand side form the spheroidal part of $\mathbf{s}$ and the last term is the toroidal part of $\mathbf{s}$. A toroidal vector field is divergence-free and perpendicular to the position vector $\mathbf{r}$. Using the identities

$\nabla \times(f \mathbf{r})=f \nabla \times \mathbf{r}-\mathbf{r} \times \nabla f$

and

$\nabla \times \mathbf{r}=0$,

we find that the contravariant components of $\mathbf{s}$ are

$s^{q}=\frac{1}{1+\frac{\partial h}{\partial q}}\left[U+\frac{1}{q+h} \frac{\partial h}{\partial \chi}\left(-\frac{\partial V}{\partial \chi}+\frac{1}{1+\frac{\partial h}{\partial q}} \frac{\partial h}{\partial \chi} \frac{\partial V}{\partial q}-\frac{1}{\sin \chi} \frac{\partial W}{\partial v}\right)\right]$,

$s^{\chi}=\frac{1}{q+h}\left(\frac{\partial V}{\partial \chi}-\frac{1}{1+\frac{\partial h}{\partial q}} \frac{\partial h}{\partial \chi} \frac{\partial V}{\partial q}+\frac{1}{\sin \chi} \frac{\partial W}{\partial v}\right)$,

$s^{v}=\frac{1}{q+h} \frac{1}{\sin \chi}\left(\frac{1}{\sin \chi} \frac{\partial V}{\partial v}-\frac{\partial W}{\partial \chi}+\frac{1}{1+\frac{\partial h}{\partial q}} \frac{\partial h}{\partial \chi} \frac{\partial W}{\partial q}\right)$.

By index-lowering, we obtain the covariant components of $\mathbf{s}$ :

$s_{i}=g_{i j} s^{j}$.

Explicitly,

$s_{q}=\left(1+\frac{\partial h}{\partial q}\right) U$

$s_{\chi}=\frac{\partial h}{\partial \chi} U+(q+h)\left(\frac{\partial V}{\partial \chi}-\frac{1}{1+\frac{\partial h}{\partial q}} \frac{\partial h}{\partial \chi} \frac{\partial V}{\partial q}+\frac{1}{\sin \chi} \frac{\partial W}{\partial v}\right)$,

$s_{v}=(q+h) \sin \chi\left(\frac{1}{\sin \chi} \frac{\partial V}{\partial v}-\frac{\partial W}{\partial \chi}+\frac{1}{1+\frac{\partial h}{\partial q}} \frac{\partial h}{\partial \chi} \frac{\partial W}{\partial q}\right)$.

The divergence of $\mathbf{s}$ is

$$
\begin{aligned}
\boldsymbol{\nabla} \cdot \mathbf{s}= & \frac{1}{1+\frac{\partial h}{\partial q}} \frac{\partial U}{\partial q}+\frac{1}{(q+h)}\left[2 U+\frac{1}{\left(1+\frac{\partial h}{\partial q}\right)^{2}}\left(\frac{\partial h}{\partial \chi}\right)^{2} \frac{\partial^{2} V}{\partial q^{2}}-\frac{2}{1+\frac{\partial h}{\partial q}} \frac{\partial h}{\partial \chi} \frac{\partial^{2} V}{\partial \chi \partial q}\right] \\
& +\frac{1}{(q+h)} \frac{1}{1+\frac{\partial h}{\partial q}}\left\{-\frac{\partial^{2} h}{\partial \chi^{2}}+\left[-\frac{\cos \chi}{\sin \chi}+\frac{2}{1+\frac{\partial h}{\partial q}} \frac{\partial^{2} h}{\partial \chi \partial q}-\frac{\frac{\partial^{2} h}{\partial q^{2}}}{\left(1+\frac{\partial h}{\partial q}\right)^{2}} \frac{\partial h}{\partial \chi}\right] \frac{\partial h}{\partial \chi}\right\} \frac{\partial V}{\partial q} \\
& +\frac{1}{(q+h)}\left(\frac{\partial^{2} V}{\partial \chi^{2}}+\frac{\cos \chi}{\sin \chi} \frac{\partial V}{\partial \chi}+\frac{1}{\sin ^{2} \chi} \frac{\partial^{2} V}{\partial v^{2}}\right) .
\end{aligned}
$$

\subsection{Strain tensor}

If $\mathbf{s}$ denotes the infinitesimal displacement vector, the linearized strain tensor $\boldsymbol{e}$ has covariant components given by

$e_{i j}=\frac{1}{2}\left(s_{i ; j}+s_{j ; i}\right)=\frac{1}{2}\left(s_{i, j}+s_{j, i}-2 \Gamma_{i j}^{k} s_{k}\right)$.

Define

$F_{(\chi)}=\frac{\partial V}{\partial \chi}-\frac{1}{1+\frac{\partial h}{\partial q}} \frac{\partial h}{\partial \chi} \frac{\partial V}{\partial q}+\frac{1}{\sin \chi} \frac{\partial W}{\partial v}$ 
and

$F_{(v)}=\frac{1}{\sin \chi} \frac{\partial V}{\partial v}-\frac{\partial W}{\partial \chi}+\frac{1}{1+\frac{\partial h}{\partial q}} \frac{\partial h}{\partial \chi} \frac{\partial W}{\partial q}$.

Consequently,

$$
\begin{aligned}
& \frac{\partial F_{(\chi)}}{\partial q}=\frac{\partial^{2} V}{\partial \chi \partial q}+\frac{1}{\left(1+\frac{\partial h}{\partial q}\right)^{2}} \frac{\partial^{2} h}{\partial q^{2}} \frac{\partial h}{\partial \chi} \frac{\partial V}{\partial q}-\frac{1}{1+\frac{\partial h}{\partial q}} \frac{\partial^{2} h}{\partial \chi \partial q} \frac{\partial V}{\partial q}-\frac{1}{1+\frac{\partial h}{\partial q}} \frac{\partial h}{\partial \chi} \frac{\partial^{2} V}{\partial q^{2}}+\frac{1}{\sin \chi} \frac{\partial^{2} W}{\partial q \partial v}, \\
& \frac{\partial F_{(\chi)}}{\partial \chi}=\frac{\partial^{2} V}{\partial \chi^{2}}+\frac{1}{\left(1+\frac{\partial h}{\partial q}\right)^{2}} \frac{\partial^{2} h}{\partial \chi \partial q} \frac{\partial h}{\partial \chi} \frac{\partial V}{\partial q}-\frac{1}{1+\frac{\partial h}{\partial q}} \frac{\partial^{2} h}{\partial \chi^{2}} \frac{\partial V}{\partial q}-\frac{1}{1+\frac{\partial h}{\partial q}} \frac{\partial h}{\partial \chi} \frac{\partial^{2} V}{\partial q \partial \chi}-\frac{\cos \chi}{\sin ^{2} \chi} \frac{\partial W}{\partial v}+\frac{1}{\sin \chi} \frac{\partial^{2} W}{\partial \chi \partial v}, \\
& \frac{\partial F_{(\chi)}}{\partial v}=\frac{\partial^{2} V}{\partial \chi \partial v}-\frac{1}{1+\frac{\partial h}{\partial q}} \frac{\partial h}{\partial \chi} \frac{\partial^{2} V}{\partial q \partial v}+\frac{1}{\sin \chi} \frac{\partial^{2} W}{\partial v^{2}}, \\
& \frac{\partial F_{(v)}}{\partial q}=\frac{1}{1+\frac{\partial h}{\partial q}} \frac{\partial h}{\partial \chi} \frac{\partial^{2} W}{\partial q^{2}}-\frac{\partial^{2} W}{\partial q \partial \chi}-\frac{1}{\left(1+\frac{\partial h}{\partial q}\right)^{2}} \frac{\partial^{2} h}{\partial q^{2}} \frac{\partial h}{\partial \chi} \frac{\partial W}{\partial q}+\frac{1}{1+\frac{\partial h}{\partial q}} \frac{\partial^{2} h}{\partial \chi \partial q} \frac{\partial W}{\partial q}+\frac{1}{\sin \chi} \frac{\partial^{2} V}{\partial v \partial q}, \\
& \frac{\partial F_{(v)}}{\partial \chi}=\frac{1}{1+\frac{\partial h}{\partial q}} \frac{\partial^{2} h}{\partial \chi^{2}} \frac{\partial W}{\partial q}+\frac{1}{1+\frac{\partial h}{\partial q}} \frac{\partial h}{\partial \chi} \frac{\partial^{2} W}{\partial q \partial \chi}-\frac{\partial^{2} W}{\partial \chi^{2}}-\frac{1}{\left(1+\frac{\partial h}{\partial q}\right)^{2}} \frac{\partial^{2} h}{\partial q \partial \chi} \frac{\partial h}{\partial \chi} \frac{\partial W}{\partial q}-\frac{\cos \chi}{\sin { }^{2} \chi} \frac{\partial V}{\partial v}+\frac{1}{\sin \chi} \frac{\partial^{2} V}{\partial \chi \partial v}, \\
& \frac{\partial F_{(v)}}{\partial v}=\frac{1}{\sin \chi} \frac{\partial^{2} V}{\partial v^{2}}-\frac{\partial^{2} W}{\partial v \partial \chi}+\frac{1}{1+\frac{\partial h}{\partial q}} \frac{\partial h}{\partial \chi} \frac{\partial^{2} W}{\partial v \partial q} \cdot
\end{aligned}
$$

The covariant components of the strain tensor are then

$$
\begin{aligned}
& e_{q q}=\left(1+\frac{\partial h}{\partial q}\right) \frac{\partial U}{\partial q}, \\
& e_{q \chi}=\frac{1}{2}\left(1+\frac{\partial h}{\partial q}\right)\left(\frac{\partial U}{\partial \chi}-F_{(\chi)}\right)+\frac{1}{2} \frac{\partial h}{\partial \chi} \frac{\partial U}{\partial q}+\frac{1}{2}(q+h) \frac{\partial F_{(\chi)}}{\partial q}, \\
& e_{q v}=\frac{1}{2}\left(1+\frac{\partial h}{\partial q}\right)\left(\frac{\partial U}{\partial \nu}-\sin \chi F_{(v)}\right)+\frac{1}{2}(q+h) \sin \chi \frac{\partial F_{(v)}}{\partial q}, \\
& e_{\chi \chi}=(q+h)\left(U+\frac{\partial F_{(\chi)}}{\partial \chi}\right)-\frac{\partial h}{\partial \chi} F_{(\chi)}, \\
& e_{\chi v}=\frac{1}{2}\left\{\frac{\partial h}{\partial \chi} \frac{\partial U}{\partial v}-\left[\frac{\partial h}{\partial \chi} \sin \chi+(q+h) \cos \chi\right] F_{(v)}+(q+h)\left(\frac{\partial F_{(\chi)}}{\partial v}+\sin \chi \frac{\partial F_{(v)}}{\partial \chi}\right)\right\}, \\
& e_{v v}=(q+h) \sin \chi\left(\sin \chi U+\cos \chi F_{(\chi)}+\frac{\partial F_{(v)}}{\partial v}\right) .
\end{aligned}
$$

By index-raising twice, we obtain the contravariant components of the strain tensor:

$$
e^{q q}=\frac{1}{\left(1+\frac{\partial h}{\partial q}\right)^{2}(q+h)^{2}}\left\{\frac{(q+h)^{2}+\left(\frac{\partial h}{\partial \chi}\right)^{2}}{\left(1+\frac{\partial h}{\partial q}\right)} \frac{\partial U}{\partial q}+\frac{\partial h}{\partial \chi}\left[-\frac{3}{2} \frac{\partial U}{\partial \chi}+F_{(\chi)}+\frac{1}{(q+h)} \frac{\partial h}{\partial \chi}\left(U+\frac{\partial F_{(\chi)}}{\partial \chi}\right)-\frac{(q+h)^{2}+\left(\frac{\partial h}{\partial \chi}\right)^{2}}{(q+h)\left(1+\frac{\partial h}{\partial q}\right)} \frac{\partial F_{(\chi)}}{\partial q}\right]\right\},
$$$$
e^{q \chi}=\frac{1}{\left(1+\frac{\partial h}{\partial q}\right)(q+h)^{2}}\left\{-\frac{\partial h}{\partial \chi}\left[\frac{1}{2} \frac{1}{\left(1+\frac{\partial h}{\partial q}\right)} \frac{\partial U}{\partial q}+\frac{1}{(q+h)}\left(U+\frac{\partial F_{(\chi)}}{\partial \chi}\right)\right]+\frac{\frac{1}{2}(q+h)^{2}+\left(\frac{\partial h}{\partial \chi}\right)^{2}}{(q+h)\left(1+\frac{\partial h}{\partial q}\right)} \frac{\partial F_{(\chi)}}{\partial q}+\frac{1}{2}\left(\frac{\partial U}{\partial \chi}-F_{(\chi)}\right)\right\}
$$

$e^{\chi \chi}=\frac{1}{(q+h)^{3}}\left\{U-\frac{1}{\left(1+\frac{\partial h}{\partial q}\right)} \frac{\partial h}{\partial \chi} \frac{\partial F_{(\chi)}}{\partial q}+\frac{\partial F_{(\chi)}}{\partial \chi}\right\}$, 


$$
\begin{aligned}
e^{q v}= & \frac{1}{2\left(1+\frac{\partial h}{\partial q}\right)(q+h)^{2} \sin ^{2} \chi}\left\{\frac{\partial U}{\partial v}+\left[\frac{\cos \chi}{(q+h)} \frac{\partial h}{\partial \chi}-\sin \chi\right] F_{(v)}\right. \\
& \left.+\frac{1}{(q+h)}\left[\left[(q+h)^{2}+\left(\frac{\partial h}{\partial \chi}\right)^{2}\right] \frac{\sin \chi}{\left(1+\frac{\partial h}{\partial q}\right)} \frac{\partial F_{(v)}}{\partial q}-\frac{\partial h}{\partial \chi}\left(\frac{\partial F_{(\chi)}}{\partial v}+\sin \chi \frac{\partial F_{(v)}}{\partial \chi}\right)\right]\right\}, \\
e^{\chi v}= & \frac{1}{2(q+h)^{3} \sin ^{2} \chi}\left[-\cos \chi F_{(v)}-\frac{\sin \chi}{\left(1+\frac{\partial h}{\partial q}\right)} \frac{\partial h}{\partial \chi} \frac{\partial F_{(v)}}{\partial q}+\frac{\partial F_{(\chi)}}{\partial v}+\sin \chi \frac{\partial F_{(v)}}{\partial \chi}\right], \\
e^{v v}= & \frac{1}{(q+h)^{3} \sin ^{3} \chi}\left(\sin \chi U+\cos \chi F_{(\chi)}+\frac{\partial F_{(v)}}{\partial v}\right)
\end{aligned}
$$

\subsection{Divergence of a tensor field}

If $\boldsymbol{t}$ is a differentiable second-order tensor function of $q, \chi$ and $v$, its divergence is the vector $\boldsymbol{\nabla} \cdot \boldsymbol{t}$ whose $i$ contravariant component is

$[\nabla \cdot \boldsymbol{t}]^{i}=t_{; j}^{i j}=t_{, j}^{i j}+\Gamma_{k j}^{i} t^{k j}+\Gamma_{j k}^{j} t^{i k}$.

Therefore,

$$
\begin{aligned}
& {[\boldsymbol{\nabla} \cdot \boldsymbol{t}]^{q}=\frac{\partial t^{q q}}{\partial q}+\frac{\partial t^{q \chi}}{\partial \chi}+\frac{\partial t^{q \nu}}{\partial v}+2\left(\Gamma_{q q}^{q}+\Gamma_{q \chi}^{\chi}\right) t^{q q}+\left(3 \Gamma_{q \chi}^{q}+\Gamma_{\chi \chi}^{\chi}+\Gamma_{\chi \nu}^{v}\right) t^{q \chi}+\Gamma_{\chi \chi}^{q} t^{\chi \chi}+\Gamma_{\nu v}^{q} t^{\nu v}} \\
& {[\nabla \cdot \boldsymbol{t}]^{\chi}=\frac{\partial t^{\chi q}}{\partial q}+\frac{\partial t^{\chi \chi}}{\partial \chi}+\frac{\partial t^{\chi v}}{\partial v}+\left(3 \Gamma_{\chi q}^{\chi}+\Gamma_{q q}^{q}+\Gamma_{v q}^{v}\right) t^{\chi q}+\left(2 \Gamma_{\chi \chi}^{\chi}+\Gamma_{q \chi}^{q}+\Gamma_{\chi v}^{v}\right) t^{\chi \chi}+\Gamma_{v v}^{\chi} v^{v v}} \\
& {[\boldsymbol{\nabla} \cdot \boldsymbol{t}]^{\nu}=\frac{\partial t^{\nu q}}{\partial q}+\frac{\partial t^{\nu \chi}}{\partial \chi}+\frac{\partial t^{\nu v}}{\partial v}+\left(3 \Gamma_{\chi \nu}^{\nu}+\Gamma_{q \chi}^{q}+\Gamma_{\chi \chi}^{\chi}\right) t^{\nu \chi}+\left(4 \Gamma_{v q}^{v}+\Gamma_{q q}^{q}\right) t^{\nu q} .}
\end{aligned}
$$

The formulae established in this section and in Section 2 are exact in $h$, but no expression has been given yet for $h$. We recall that $h$ is the radial distance between the position of a particle in the spherical configuration of reference and its position in the deformed state. The deformation of the spherical model is due to the uniform rotation of the body. To determine $h$, we must make assumptions as to the rheological behaviour of the body. We will suppose that on very long timescales the Earth behaves like an incompressible liquid body. Therefore, the rotating Earth is supposed to be in hydrostatic equilibrium in a rotating frame of reference. The next section is devoted to the computation of $h$ by means of the theory of hydrostatic figures of equilibrium.

\section{HYDROSTATIC FIGURES OF EQUILIBRIUM}

The rotating body being in a state of hydrostatic equilibrium, the function $h(q, \chi)$ is determined as soon as the density profile $\rho_{0}(q)$ of the corresponding spherically averaged earth model and its constant angular speed of rotation $\Omega_{0}=\Omega_{0} \mathbf{e}_{z}$ are known. We show how in this section. Our computations rely on the theory of hydrostatic figures of equilibrium (e.g. Zharkov \& Trubitsyn 1978, pp. 221-295; Denis 1989; Denis et al. 1998).

We consider a system of reference rotating at a constant angular speed $\boldsymbol{\Omega}_{0}$ about its $z$-axis, which is fixed in absolute space. The earth also rotates steadily about the $z$-axis at the angular speed $\Omega_{0}$. The equation of hydrostatic equilibrium is

$\nabla p=-\rho \nabla \tilde{\phi}$,

where $p$ is the pressure and $\tilde{\phi}$ is the gravity potential given by

$\tilde{\phi}(\mathbf{r})=\phi(\mathbf{r})-\frac{1}{2}\left|\Omega_{0} \times \mathbf{r}\right|^{2}$.

The gravitational potential $\phi$ at $\mathbf{r}$ is

$\phi(\mathbf{r})=-\mathrm{G} \int_{\mathcal{V}} \frac{\rho\left(\mathbf{r}^{\prime}\right)}{\left|\mathbf{r}-\mathbf{r}^{\prime}\right|} d \mathcal{V}^{\prime}$.

Of course, $\rho$ is the density, $\mathrm{G}$ is the universal constant of gravitation, and $\mathcal{V}$ designates the volume occupied by the rotating earth. Then, $\tilde{\phi}$ is a solution of Poisson's equation:

$\nabla^{2} \tilde{\phi}=4 \pi \mathrm{G} \rho-2 \Omega_{0}^{2}$. 
It can be shown that eq. (76) implies that a surface of constant pressure coincides with an equipotential surface and that it is a surface of constant density. Our goal is to establish the equation of the equisurfaces under the form of eq. (1), knowing the density profile of a spherically averaged reference model. Because of the axial symmetry of the rotating body, $h$ does not depend on $v$, in agreement with eq. (1). We seek for $h$ a solution that is a series of Legendre polynomials of $\cos \chi$ :

$h(q, \chi)=\sum_{\ell=0}^{\infty} h_{2 \ell}(q) P_{2 \ell}(\cos \chi)$

where $P_{\ell}(x)$ is the Legendre polynomial of degree $\ell$ (see Appendix A1). Because of the symmetry of the rotating body with respect to the equatorial plane, series (80) does not contain terms of odd degree. Now, we have to determine the figure functions $h_{2 \ell}(q)$.

Let us denote by $\rho_{0}(q)$ the density profile of a spherical earth model and by $\rho^{0}(r, \theta)$ the density of the corresponding rotating model as a function of the spherical coordinates. Since we consider a Lagrangian perturbation of the spherical model with no density change, we have

$\rho^{0}[r(q, \chi), \theta(\chi)]=\rho_{0}(q)$

We have seen that the equidensity, equipressure and equipotential surfaces of the rotating model coincide. Therefore, the pressure and the gravity potential depend only on $q$ when they are written as functions of the coordinates $(q, \chi, v)$. Let us denote these functions by $p_{0}(q)$ and $\tilde{\phi}_{0}(q)$, respectively. The figure functions $h_{2 \ell}$ are then determined in the following way: eq. (77) is written in the coordinates $(q, \chi, v)$ and we require that $\tilde{\phi}_{0}$ does not depend on $\chi$ and $\nu$. We also take into account the fact that the spherical model and the rotating model enclose the same volume.

In practice, we limit the expansion of $h$ to three terms:

$h=h_{0}+h_{2} P_{2}+h_{4} P_{4}$,

where $P_{2}$ and $P_{4}$ are given in the Appendix A4. The second term is dominant because the dominant term in the rotational potential $-\mid \Omega_{0}$ $\times\left.\mathbf{r}\right|^{2} / 2$, expressed as a function of $q$ and $\chi$, is the second-degree term. Moreover, $\left|h_{2}\right|$ is obviously expected to be of the order of $\Omega_{0}^{2} q / \mathrm{G} \bar{\rho}$, where $\bar{\rho}$ is the mean density of the sphere of radius $q$ :

$\bar{\rho}(q)=\frac{3}{q^{3}} \int_{0}^{q} \rho_{0} q^{\prime 2} d q^{\prime}$

The zeroth-degree term in the rotational potential is of the same order as the second-degree term, but $h_{0}$ is much smaller than $h_{2}$ because we assume that the Lagrangian perturbation is incompressible. It can indeed be shown that the conservation of volume gives

$h_{0}=-\frac{h_{2}^{2}}{5 q}$.

Moreover, we will show that $h_{4} / q$ is of the order of $\left(h_{2} / q\right)^{2}$. More generally, we would have $h_{2 \ell} / q=\mathcal{O}\left[\left(h_{2} / q\right)^{\ell}\right]$ for $\ell \geq 1$, where the symbol $\mathcal{O}(x)$ means 'of the order of $x$ '.

Let us now write the rotational potential and the gravitational potential $\phi$ in terms of $q$ and $\chi$. For the former we have, to second order in $h_{2} / q$,

$-\frac{1}{2}\left|\Omega_{0} \times \mathbf{r}\right|^{2}=-\frac{1}{3} \Omega_{0}^{2} q^{2}\left[1-\frac{2}{5} \frac{h_{2}}{q}-\left(1-\frac{10}{7} \frac{h_{2}}{q}\right) P_{2}-\frac{36}{35} \frac{h_{2}}{q} P_{4}\right]$,

where we have used eq. (A23). Next, eq. (78), formulae listed in Appendix A4, and the well-known expansion of $1 /\left|\mathbf{r}-\mathbf{r}^{\prime}\right|$ in series of spherical harmonics $\mathcal{D}_{\ell}^{m}$ defined in Appendix A1 (the star denotes complex conjugation), namely

$$
\begin{aligned}
\frac{1}{\left|\mathbf{r}-\mathbf{r}^{\prime}\right|} & =\frac{1}{r} \sum_{\ell=0}^{\infty}\left(\frac{r^{\prime}}{r}\right)^{\ell} \sum_{m=-\ell}^{\ell} \mathcal{D}_{\ell}^{m *}\left(\chi^{\prime}, v^{\prime}\right) \mathcal{D}_{\ell}^{m}(\chi, v) \quad \text { if } \quad r^{\prime}<r \\
& =\frac{1}{r^{\prime}} \sum_{\ell=0}^{\infty}\left(\frac{r}{r^{\prime}}\right)^{\ell} \sum_{m=-\ell}^{\ell} \mathcal{D}_{\ell}^{m *}\left(\chi^{\prime}, v^{\prime}\right) \mathcal{D}_{\ell}^{m}(\chi, v) \quad \text { if } \quad r^{\prime}>r,
\end{aligned}
$$


give

$\phi_{0}(q, \chi)=-4 \pi \mathrm{G}\left\{\frac{1}{q} \int_{0}^{q} \rho_{0} q^{\prime 2}\left[1+\frac{2 h_{0}}{q^{\prime}}+\frac{d h_{0}}{d q^{\prime}}+\frac{1}{5}\left(\frac{2 h_{2}}{q^{\prime}} \frac{d h_{2}}{d q^{\prime}}+\frac{h_{2}^{2}}{q^{\prime 2}}\right)\right] d q^{\prime}\right.$

$$
\begin{aligned}
& -\frac{1}{q}\left[\frac{h_{0}}{q}+\frac{h_{2}}{q} P_{2}+\frac{h_{4}}{q} P_{4}-\frac{h_{2}^{2}}{q^{2}}\left(\frac{1}{5}+\frac{2}{7} P_{2}+\frac{18}{35} P_{4}\right)\right] \int_{0}^{q} \rho_{0} q^{\prime 2} d q^{\prime} \\
& +\frac{1}{5 q^{3}}\left[P_{2}-\frac{3 h_{2}}{q}\left(\frac{1}{5}+\frac{2}{7} P_{2}+\frac{18}{35} P_{4}\right)\right] \\
& +\frac{P_{4}}{9 q^{5}} \int_{0}^{q} \rho_{0} q^{\prime 6}\left[6 \frac{h_{4}}{q^{\prime}}+\frac{d h_{4}}{d q^{\prime}}+\frac{18}{35}\left(\frac{6 h_{2}}{q^{\prime}} \frac{d h_{2}}{d q^{\prime}}+\frac{10 h_{2}^{2}}{q^{\prime 2}}\right)\right] d q^{\prime} \\
& +\int_{q}^{R} \rho_{0} q^{\prime}\left(1+\frac{h_{2}}{d q^{\prime}}+\frac{2}{7}\left(\frac{4 h_{2}}{q^{\prime}} \frac{d h_{2}}{d q^{\prime}}+\frac{6 h_{2}^{2}}{q^{\prime 2}}\right)\right] d q^{\prime} \\
& +\frac{q^{\prime}}{5}\left[P_{2}+\frac{1}{5} \frac{h_{2}}{q^{\prime}} \frac{d h_{2}}{d q^{\prime}}\right) d q^{\prime} \\
& \left.\left.\int_{q}^{R} \frac{\rho_{0}}{q^{\prime}}\left[\frac{d h_{2}}{d q^{\prime}}-\frac{h_{2}}{q^{\prime}}+\frac{2}{7}\left(\frac{h_{2}^{2}}{q^{\prime 2}}-\frac{2}{q^{\prime}} \frac{h_{2}}{d q^{\prime}}\right)\right] d q^{\prime}+\frac{18}{35} P_{4}\right)\right] \\
& \left.+\frac{q^{4} P_{4}}{9} \int_{q}^{R} \frac{\rho_{0}}{q^{\prime 3}}\left[\frac{d h_{4}}{d q^{\prime}}-3 \frac{h_{4}}{q^{\prime}}+\frac{54}{35}\left(\frac{2 h_{2}^{2}}{q^{\prime 2}}-\frac{h_{2}}{q^{\prime}} \frac{d h_{2}}{d q^{\prime}}\right)\right] d q^{\prime}\right\}
\end{aligned}
$$

where the Legendre polynomials $P_{\ell}$ are functions of $\cos \chi$. As the gravity potential $\tilde{\phi}_{0}$, which is the sum of the gravitational potential and of the rotational potential, does not depend on $\chi$, we obtain, after some tedious manipulations of eqs (85) and (87),

$$
\begin{aligned}
& {\left[\bar{\rho}\left(1-\frac{4}{7} \frac{d h_{2}}{d q}+\frac{8}{7} \frac{h_{2}}{q}\right)+\frac{\Omega_{0}^{2}}{2 \pi \mathrm{G}}\right] q \frac{d^{2} h_{2}}{d q^{2}}+\left[\left(6 \rho_{0}-2 \bar{\rho}\right)+\left(48 \rho_{0}-100 \bar{\rho}\right) \frac{h_{2}}{7 q}+\frac{1}{7}\left(4 \bar{\rho}-6 \rho_{0}\right) \frac{d h_{2}}{d q}-\frac{2 \Omega_{0}^{2}}{\pi \mathrm{G}}\right] \frac{d h_{2}}{d q}} \\
& \quad-\left(4 \bar{\rho}+18 \rho_{0} \frac{h_{2}}{q}+\frac{2 \Omega_{0}^{2}}{\pi \mathrm{G}}\right) \frac{h_{2}}{q}=0
\end{aligned}
$$

and

$$
\begin{gathered}
\bar{\rho} q \frac{d^{2} h_{4}}{d q^{2}}+\left(6 \rho_{0}-2 \bar{\rho}\right) \frac{d h_{4}}{d q}-18 \bar{\rho} \frac{h_{4}}{q} \\
+\frac{54}{35} \rho_{0}\left[\frac{4 h_{2}^{2}}{q^{2}}+\left(\frac{d h_{2}}{d q}\right)^{2}\right]+\frac{36}{5} \bar{\rho}\left(\frac{h_{2}^{2}}{q^{2}}+\frac{4}{7} \frac{h_{2}}{q} \frac{d h_{2}}{d q}-\frac{h_{2}}{7} \frac{d^{2} h_{2}}{d q^{2}}\right) \\
+\frac{54}{175}\left[\frac{3}{q^{3}}\left(q \frac{d^{2} h_{2}}{d q^{2}}-6 \frac{d h_{2}}{d q}-8 \frac{h_{2}}{q}\right) \int_{0}^{q} \rho_{0} q^{\prime 4}\left(\frac{4 h_{2}}{q^{\prime}}+\frac{d h_{2}}{d q^{\prime}}\right) d q^{\prime}\right. \\
\left.-2\left(q \frac{d^{2} h_{2}}{d q^{2}}+4 \frac{d h_{2}}{d q}-18 \frac{h_{2}}{q}\right) \int_{q}^{R} \frac{\rho_{0}}{q^{\prime}}\left(\frac{d h_{2}}{d q^{\prime}}-\frac{h_{2}}{q^{\prime}}\right) d q^{\prime}\right] \\
+\frac{9}{35} \frac{\Omega_{0}^{2}}{\pi \mathrm{G}}\left(q \frac{d^{2} h_{2}}{d q^{2}}+4 \frac{d h_{2}}{d q}-18 \frac{h_{2}}{q}\right)=0 .
\end{gathered}
$$

In the same way, the associated boundary conditions at the surface $q=R$ are shown to be

$\frac{d h_{2}}{d q}+\frac{h_{2}}{R}-\frac{46}{7} \frac{h_{2}^{2}}{R^{2}}-\frac{2}{7} \frac{h_{2}}{R} \frac{d h_{2}}{d q}+\frac{\Omega_{0}^{2}}{4 \pi \mathrm{G} \bar{\rho}}\left(5+\frac{24}{7} \frac{d h_{2}}{d q}-\frac{94}{7} \frac{h_{2}}{R}\right)=0$ 
and

$\frac{h_{4}}{R}+\frac{1}{3} \frac{d h_{4}}{d q}-\frac{12}{35} \frac{h_{2}}{R}\left(\frac{h_{2}}{R}+\frac{d h_{2}}{d q}\right)+\frac{54}{175 R^{5}}\left(\frac{h_{2}}{R}+\frac{d h_{2}}{d q}\right) \int_{0}^{R} \rho_{0} q^{\prime 4}\left(4 \frac{h_{2}}{q^{\prime}}+\frac{d h_{2}}{d q^{\prime}}\right) d q^{\prime}+\frac{12}{35} \frac{\Omega_{0}^{2}}{4 \pi \mathrm{G} \bar{\rho}}\left(\frac{6 h_{2}}{R}+\frac{d h_{2}}{d q}\right)=0$.

Moreover, $h_{2}$ and $h_{4}$ must be finite at $q=0$.

Since the second-order ordinary differential equation (88) and boundary condition (90) do not contain $h_{4}$, they can be solved first to obtain the figure function $h_{2}(q)$. If only a first-order approximation is needed, we neglect the terms of second order in $h_{2}$ and products of $\Omega^{2}{ }_{0}$ and $h_{2}$ or its derivatives. Eq. (88) then becomes a homogeneous differential equation and boundary condition (90) shows that indeed $h_{2}=\mathcal{O}\left(-\Omega_{0}^{2} q / \pi \mathrm{G} \bar{\rho}\right)$.

Once $h_{2}$ has been computed, the second-order differential equation (89), boundary condition (91), and function $h_{2}(q)$ determine the figure function $h_{4}(q)$. The inhomogeneous terms in (89) and (91) are of the order of $\left(h_{2} / q\right)^{2}$. Therefore, $h_{4} / q=\mathcal{O}\left[\left(h_{2} / q\right)^{2}\right]$ at most. Since $h_{0}$ is given by eq. (82), the equation of the equisurfaces is now determined up to the second order. For example, in the case of a homogeneous body, we readily find that

$h_{2}=-\frac{5 \Omega_{0}^{2}}{8 \pi \mathrm{G} \rho_{0}} q$

and

$h_{4}=\frac{3}{35} \frac{h_{2}^{2}}{q}$.

Taking the mean density of the Earth to be $\rho_{0}=5515 \mathrm{~kg} \mathrm{~m}^{-3}$, we find that $h_{2} / q \simeq-0.0029$.

In this section, we have computed the equilibrium shape of the rotating body up to the second order. In the next section, we consider a small perturbation to the state of steady rotation and we write the linearized vector equations of motion. We also write the boundary conditions both at a welded interface and at a sliding interface.

\section{EQUATIONS OF MOTION FOR A ROTATING PLANET IN HYDROSTATIC EQUILIB R I U M}

A mass element of a slowly rotating body that is initially in hydrostatic equilibrium obeys the following linearized equation of linear momentum conservation:

$\rho \frac{d^{2} \mathbf{s}}{d t^{2}}=\mathbf{f}_{\mathrm{ext}}+\nabla \cdot \delta \boldsymbol{t}-\rho \nabla(\Delta \phi)+\rho(\nabla \cdot \mathbf{s}) \nabla \tilde{\phi}-\rho \nabla(\mathbf{s} \cdot \nabla \tilde{\phi})-2 \rho \boldsymbol{\Omega}_{0} \times \frac{d \mathbf{s}}{d t}$.

Here, $\mathbf{s}$ is the small displacement vector of the mass element from $\mathbf{r}, \Delta \phi$ is the Eulerian variation of the gravitational potential $\phi, \delta \boldsymbol{t}$ is the Lagrangian variation of the Cauchy stress tensor, and $\mathbf{f}_{\text {ext }}$ is the sum of all the external volume forces. We recall that $\rho$ is the equilibrium density of the mass element initially at $\mathbf{r}, \tilde{\phi}$ is the equilibrium gravity potential at $\mathbf{r}$, and $\Omega_{0}=\Omega_{0} \mathbf{e}_{z}$ is the constant angular velocity of the reference system. As we are interested in the normal modes of the Earth, we will not consider the total external force $\mathbf{f}_{\text {ext }}$ further. We also know that $\tilde{\phi}$ and $\phi$ are given by (77) and (78), respectively, and that $\Delta \phi$ must then satisfy Poisson's equation

$\nabla^{2}(\Delta \phi)=-4 \pi \mathrm{G} \nabla \cdot(\rho \mathbf{s})$.

In the vector equation of motion, we have neglected the electromagnetic Lorentz force and, in the Cauchy stress tensor, we will neglect any viscous stress. In addition, we will suppose that the stress-strain relation is isotropic elastic. Therefore, the Lagrangian variation of the Cauchy stress tensor $\boldsymbol{t}$ reads

$\delta \boldsymbol{t}=\lambda(\nabla \cdot \mathbf{s}) \mathrm{g}+2 \mu \boldsymbol{e}$,

where $\lambda$ and $\mu$ are the Lamé parameters, $\boldsymbol{g}$ is the metric tensor, and $\boldsymbol{e}$ is the strain tensor. The rigidity $\mu$ vanishes in a fluid.

The displacement vector $\mathbf{s}$, the Lagrangian variation of the Cauchy stress tensor $\delta \boldsymbol{t}$, and the Eulerian variation of the gravitational potential $\Delta \phi$ are subject to continuity conditions at those interfaces where the parameters of the model are discontinuous (Dahlen 1972). If $\mathbf{n}$ denotes the outward unit normal to any interface, the quantities $\mathbf{n} \cdot \mathbf{s}, \mathbf{n} \cdot \delta \boldsymbol{t}, \Delta \phi$, and $\mathbf{n} \cdot[\boldsymbol{\nabla}(\Delta \phi)+4 \pi \mathrm{G} \rho \mathbf{s}]$ must be continuous across that boundary. Furthermore, if the boundary is a solid-solid interface, it is welded and $\mathbf{s}$ must be continuous across the boundary. Because we neglect viscous effects, a fluid-fluid or a fluid-solid interface may be sliding. Therefore, only the normal component of the displacement must be continuous there.

In order to transform the vector partial differential equation (94) and the scalar partial differential equation (95) into a set of scalar ordinary differential equations, we first use the Helmholtz representation (40) for the displacement vector $\mathbf{s}$ and for the traction $\mathbf{n} \cdot \delta \boldsymbol{t}$. Next, we write the equation of motion (94) and Poisson's equation (95) in the curvilinear coordinates $(q, \chi, v)$ and in the frequency domain. Finally, we expand the unknown scalars on the functional basis of the surface spherical harmonics. Symmetry considerations (Smith 1974) show that the Coriolis force and the shape of the rotating body couple the spheroidal displacements with the toroidal displacements under the following rules:

$\mathbf{s}=\sum_{\ell=|m|,|m|+2, \ldots}^{\infty}\left(\boldsymbol{\sigma}_{\ell}^{m}+\boldsymbol{\tau}_{\ell+1}^{m}\right)$ 
or

$\mathbf{s}=\sum_{\ell=|m|,|m|+2, \ldots}^{\infty}\left(\tau_{\ell}^{m}+\sigma_{\ell+1}^{m}\right)$,

where $\sigma^{m}{ }_{\ell}$ denotes a spheroidal displacement of harmonic degree $\ell$ and harmonic order $m$, and $\tau^{m}{ }_{\ell}$ denotes a toroidal displacement of degree $\ell$ and order $m$. Consequently, the set of scalar ordinary differential equations whose solution is the displacement vector is infinite.

We must determine in advance to what order in $h$ the equation of motion and Poisson's equation have to be written. For the usual seismic modes whose periods are below one hour, it is well established that a first-order approximation is sufficient (Dahlen 1968; Dahlen \& Sailor 1979; Rogister 2003). The same statement also holds for the Slichter modes, whose periods are expected to be of the order of a few hours for realistic earth models (Smith 1976; Rogister 2003). However, the computation of rotational modes requires that terms of the second order in $h$ be kept in the deformation equations (Schastok 1997; Rogister 2001). Rogister (2001) computes the hydrostatic figure and the parameters of the rotating model only to the first order in $h=h_{2} P_{2}$ but takes into account the terms containing the square of $h$ both in the equations of motion and in the boundary conditions. Schastok (1997) considers a second-order approximation for the equation of the equisurfaces of the rotating body but he does not include all the terms of order of $h^{2}{ }_{2}$ in the equations of motion.

Knowing the analytical expressions for the frequency and for the displacement of the TOM, we can insert them in the equation of motion (94) and in Poisson's equation (95). So, we can show how the TOM imposes constraints on the approximations that we can make in these equations. This is what we do in the next section. The TOM was also used as a test case in the work of Rogister (2001).

\section{CONSTRAINTS IMPOSED BY THE TILT-OVER MODE ON THE APPROXIMATIONS IN THE EQUATIONS OF MOTION}

Described in inertial space, the TOM is the steady rotation of the body about a fixed axis different from the $z$-axis of the rotating reference frame, the norms of the two angular velocities being the same. The TOM does not depend on and, therefore, yields no information on the inner structure or on the shape of the body. Nevertheless, it is of importance in astronomy because its resonant excitation by luni-solar tidal forces causes precession (Moritz \& Mueller 1987). The TOM is a solution of linearized equations (94) and (95) only if the angle $\beta$ between the $z$-axis and the axis of rotation of the planet is small. In the rotating reference frame, the proper displacement is then easily shown to be the vector $\boldsymbol{\beta} \times \mathbf{r}$, where $\boldsymbol{\beta}$ is a vector rotating in the equatorial plane at the angular speed $\Omega_{0}$ in the retrograde sense. Vector $\boldsymbol{\beta}$ is the imaginary part of $\beta e^{-i \Omega_{0} t}\left(\mathbf{e}_{x}-i \mathbf{e}_{y}\right), \mathbf{e}_{x}$ and $\mathbf{e}_{y}$ being the other two unit vectors of the rotating Cartesian reference frame Oxyz. Consequently, the eigendisplacement of the TOM is the imaginary part of the vector

$\mathbf{s}_{\mathrm{TOM}}=\beta\left[\mathbf{e}_{\chi}-i \frac{\cos \chi}{\sin \chi} \mathbf{e}_{v}-\frac{1}{1+\frac{\partial h}{\partial q}} \frac{\partial h}{\partial \chi} \mathbf{e}_{q}\right] e^{i\left(\nu-\Omega_{0} t\right)}$.

By comparing this expression with contravariant components (43), (44) and (45) of s, we find that

$U_{\mathrm{TOM}}=V_{\mathrm{TOM}}=0$

$W_{\mathrm{TOM}}=i \beta(q+h) \sin \chi e^{i\left(v-\Omega_{0} t\right)}$.

Because there is no deformation associated with the TOM, both the tensor $\delta \boldsymbol{t}$ and the divergence of the displacement vector vanish.

Let us then denote by $\underline{W}$ the Fourier transform of $W_{\text {TOM }}$ :

$$
\begin{aligned}
\underline{W}(q, \chi, v ; \omega) & =\int_{-\infty}^{+\infty} W_{\mathrm{TOM}}(q, \chi, v ; t) e^{i \omega t} d t \\
& =2 \pi i \beta(q+h) \sin \chi e^{i v} \delta\left(\omega-\Omega_{0}\right),
\end{aligned}
$$

where we have emphasized the variable-dependences of $\underline{W}$ and $W$ TOM, and where $\delta(x)$ is the delta function. Similarly, we denote by $\underline{\phi}$ the Fourier transform of $(\Delta \phi)_{\text {Tом }}$ and we expand $\underline{\phi}$ on the functional basis of the spherical harmonics $\mathcal{D}_{\ell}^{m}(\chi, \nu)$ :

$\underline{\phi}(q, \chi, v ; \omega)=2 \pi \sum_{\ell=0}^{\infty} \sum_{m=-\ell}^{\ell} \phi_{\ell}^{m}(q) \mathcal{D}_{\ell}^{m}(\chi, v) \delta\left(\omega-\Omega_{0}\right)$.

After having taken the Fourier transform of the equation of motion (94), we insert in it the solution for the TOM angular frequency, $\Omega_{0}$, and the solution for the TOM displacement. We obtain the $v$-covariant component of (94) which, after some rearrangement, reads

$\Omega_{0}^{2}(q+h)^{2} \sin ^{2} \chi\left[\left(1+\frac{\partial h}{\partial q}\right)\left(\frac{\partial \underline{W}}{\partial \chi}+2 i \frac{\cos \chi}{\sin \chi} \frac{\partial \underline{W}}{\partial v}\right)-\frac{\partial h}{\partial \chi} \frac{\partial \underline{W}}{\partial q}\right]=-(q+h) \sin \chi\left(1+\frac{\partial h}{\partial q}\right) \frac{\partial \underline{\phi}}{\partial v}+\frac{\partial h}{\partial \chi} \frac{\partial^{2} \underline{W}}{\partial v^{2}} \frac{d \tilde{\phi}_{0}}{d q}$

or

$\Omega_{0}^{2} \beta(q+h)^{2}\left(1+\frac{\partial h}{\partial q}\right) \frac{\sqrt{6}}{3} \mathcal{D}_{2}^{1}-\left(1+\frac{\partial h}{\partial q}\right) \sum_{\ell=0}^{\infty} \sum_{m=-\ell}^{\ell} m \phi_{\ell}^{m}(q) \mathcal{D}_{\ell}^{m}(\chi, v)=\beta \frac{\partial h}{\partial \chi} e^{i v} \frac{d \tilde{\phi}_{0}}{d q}$, 
where

$\mathcal{D}_{2}^{1}(\chi, v)=\frac{\sqrt{6}}{2} \cos \chi \sin \chi e^{i v}$.

In eq. (105), the right-hand side and the first term on the left-hand side being proportional to $e^{i v}$, the gravitational variation $\underline{\phi}$ must be propotional to $e^{i v}$ too. Thus, $\phi$ simplifies to

$\underline{\phi}=2 \pi \sum_{\ell=0}^{\infty} \phi_{\ell}^{1}(q) \mathcal{D}_{\ell}^{1}(\chi, v) \delta\left(\omega-\Omega_{0}\right)$.

Gravitational variations of different harmonic orders do not couple with each other, confirming what can be deduced from symmetry considerations. The functions $\phi_{\ell}^{1}(q)$ can then be determined by identification of the terms having the same harmonic degree $\ell$ in eq. (105). Expanding $h$ in spherical harmonics of order 0 (eq. 80) and invoking relations (A.10) and (A.11), as well as a symmetry property of the J-squares given in Appendix A3, we see that eq. (105) involves only surface spherical harmonics of even degree $\ell \geq 2$ and order 1 . Thus, $\phi$ becomes

$\underline{\phi}=2 \pi \sum_{\ell=1}^{\infty} \phi_{2 \ell}^{1}(q) \mathcal{D}_{2 \ell}^{1}(\chi, v) \delta\left(\omega-\Omega_{0}\right)$.

Before we start making approximations, it must be noted that eq. (105) is exact in $h$. Now, let us first consider the first-order approximation $h=h_{2} \mathcal{D}_{2}^{0}$. Identifying the terms of degree 2 and order 1 , we obtain

$\phi_{2}^{1}=\sqrt{6} \beta\left(\frac{\Omega_{0}^{2}}{3} q^{2}+h_{2} \frac{d \tilde{\phi}_{0}}{d q}\right)$,

in agreement with eq. (48) of Rogister (2001). In the same way, the identification of the terms of degree 4 shows that $\phi^{1}{ }_{4}$ is of the order of $\left(h_{2}\right)^{2}$ and is therefore negligible in a first-order approach. Coefficients $\phi_{\ell}^{1}$ with $\ell>4$ are also negligible. The important conclusion that we can draw is that, in order to obtain relation (109), the right-hand side of eq. (105) is computed to the first order in $h$ whereas the left-hand side is computed to the zeroth order in $h$.

Let us next consider the second-order approximation $h=h_{0}+h_{2} \mathcal{D}_{2}^{0}+h_{4} \mathcal{D}_{4}^{0}$. Identifying the terms of degree 2 and order 1 , we obtain

$\phi_{2}^{1}=\sqrt{6} \beta\left[\frac{\Omega_{0}^{2}}{3} q\left(q+\frac{2}{7} h_{2}\right)+h_{2}\left(1-\frac{1}{7} \frac{d h_{2}}{d q}\right) \frac{d \tilde{\phi}_{0}}{d q}\right]$,

and identifying the terms of degree 4 and order 1 , we find

$\phi_{4}^{1}=\sqrt{20} \beta\left[\frac{6}{35} \Omega_{0}^{2} q h_{2}+\left(h_{4}-\frac{9}{35} h_{2} \frac{d h_{2}}{d q}\right) \frac{d \tilde{\phi}_{0}}{d q}\right]$.

Coefficients $\phi_{\ell}^{1}$ with $\ell>4$ are negligible in a second-order approach. We see that it is necessary that $h$ on the right-hand side of eq. (105) be computed up to the second order, whereas, on the left-hand side, $h$ needs to be computed to the first order only. The reason for this is that the restoring force stemming from the gravitational attraction of the equatorial bulge contributes as much as the inertia forces and the force arising from the Eulerian gravitational variation. Indeed, we have seen in Section 4 that $h_{2}=\mathcal{O}\left(-\Omega_{0}^{2} q / \pi \mathrm{G} \bar{\rho}\right)$. Moreover, $d \tilde{\phi}_{0} / d q=\mathcal{O}(\pi \mathrm{G} \bar{\rho} q)$. Consequently, $\left|h_{2} \mathbf{s}\right| d \tilde{\phi}_{0} / d q=\mathcal{O}\left(\Omega_{0}^{2}|\mathbf{s}| q^{2}\right)$, which is of the order of the inertia forces divided by $\rho_{0}$.

It is only a matter of algebra to check that solutions (109), (110) and (111) also satisfy the $q$ - and $\chi$-covariant components of the equation of motion (94), under the condition that the approximation to which the terms involving the initial gravity are computed is one order higher than the approximation to which the inertia terms and the terms containing the gravitational variation are computed. It can also be checked that those solutions satisfy Poisson's equation (95) provided that $h$ on the right-hand side is calculated to one order higher than $h$ on the left-hand side. The rotational motions, i.e. wobbles and nutations, are characterized by a dominant rigid rotation about an equatorial axis and a much smaller deformation. Indeed, their displacement vector has the form

$\mathbf{s}=\tau_{1}^{m}+\boldsymbol{\sigma}_{2}^{m}+\tau_{3}^{m}+\boldsymbol{\sigma}_{4}^{m}+\tau_{5}^{m}+\cdots$,

where $m= \pm 1$. At the beginning of this section, we showed that the toroidal displacement of degree 1 and order \pm 1 , which is dominant for rotational motions, is a rotation about an equatorial axis. If it is a linear function of $r$, it describes a rigid rotation of the whole body about that axis. The other terms, which are elasto-gravitational deformations, are smaller than $\tau^{m}{ }_{1}$ by a factor of the order of, at most, $h_{2} / q$. Moreover, for rotational motions, the restoring force produced by the gravitational attraction of the equatorial bulge plays an essential role. These similarities between the TOM and the other rotational modes strongly suggest that the conclusions that we have drawn for the TOM regarding the approximations allowed in the equations of motion also apply to the other rotational motions. When we solve numerically the equation of motion for a rotational mode, we first make an approximation for $h$, then we search for the eigenfrequency and the eigenfunction of the mode. This is different from what we did above for the TOM: we knew the eigenfrequency and the eigendisplacement and we searched for the allowed approximation for $h$. The gravitational potential variation associated with the TOM was determined up to the second order in $h_{2} / q$ provided that $h$ was also computed to the second order in the terms involving the equilibrium gravity and provided that two terms were kept in series (108). To warrant a relative accuracy of the order of $\left(h_{2} / q\right)^{2}$ for both the eigenfrequency and the eigenfunction of a rotational mode, it is therefore necessary to include four terms in series (112) and to compute the terms involving the equilibrium gravity up to the second order. 
Since the TOM is divergence-free and it involves no deformation, it does not impose any constraint on the terms $\rho(\nabla \cdot \mathbf{s}) \nabla \tilde{\phi}$ and $\boldsymbol{\nabla} \cdot \delta \boldsymbol{t}$ in eq. (94). Thus, we may question the admissible approximation that can be applied to $h$ in these terms when we consider other rotational motions. As the term $\rho(\nabla \cdot \mathbf{s}) \nabla \tilde{\phi}$ involves the equilibrium gravity, it is consistent to compute it to the second order in $h_{2} / q$. The rigid rotation displacement contributes nothing to the elastic term given by $\nabla \cdot \delta \boldsymbol{t}$. Since the terms in (112) that contribute to the elastic force in eq. (94) are smaller than $\tau^{m}{ }_{1}$ by a factor of the order of, at most, $h_{2} / q$, it is sufficient to compute the term $\nabla \cdot \delta \boldsymbol{t}$ to the first order in $h_{2} / q$.

\section{SCALAR EQUATIONS OF MOTION FOR A ROTATING PLANET IN HYDROSTATIC EQUILIBR I UM}

In three steps, we perform the transformation of the vector equation of motion (94), of Poisson's equation (95), and of the stress-strain relation (96) into a set of first-order ordinary differential equations over radius $q$. First, we take the Fourier time transform of each of these equations, substituting multiplication by $i \omega$ for the derivative with respect to time, where $\omega$ is the frequency of the motion. Second, we decompose the vector fields into a spheroidal part and a toroidal part and use the results of Sections 3 and 6 to write the approximate equations in the coordinate system $q, \chi, v$. Third, we expand the toroidal and spheroidal scalars on the basis of the surface spherical harmonics. Generally, we do not write explicitly the second step of the calculations but directly provide the formulae for the ordinary differential equations obeyed by the harmonic components of the various scalars. These scalars are the spheroidal and the toroidal scalars of the displacement vector and the traction, the gravitational potential variation and the scalar defined by eq. (120). Because the harmonic components of the displacement vector couple following the rules (97) or (98), we obtain an infinite set of ordinary differential equations. We give the results for a solid material. The formulae for a liquid material can be obtained by letting the rigidity vanish.

\subsection{Stress-strain relation}

In coordinates $(q, \chi, v)$, the covariant components of the stress-strain relation (96) become

$\delta t_{i j}=\lambda_{0}(\nabla \cdot \mathbf{s}) g_{i j}+2 \mu_{0} e_{i j}$,

where $\lambda_{0}(q)$ and $\mu_{0}(q)$ are the Lamé parameters as functions of $q$. The Lamé parameters do not depend on $\chi$ and $v$ because we suppose that the surfaces of constant Lamé parameters coincide with the spherical surfaces of constant initial density. Introducing the scalars $P, Q$ and $R$, we define the Helmholtz representation of the vector $\mathbf{e}_{q} \cdot \delta \boldsymbol{t}$ :

$\mathbf{e}_{q} \cdot \delta \boldsymbol{t}=P \frac{\mathbf{r}}{r}+\frac{\mathbf{r}}{r} \times[\nabla \times(Q \mathbf{r})]+\nabla \times(R \mathbf{r})$.

The $q, \chi$ and $v$ covariant components of $\mathbf{e}_{q} \cdot \delta \boldsymbol{t}$ are, respectively, $\delta t_{q q}, \delta t_{q \chi}$ and $\delta t_{q v}$, and can be expressed in terms of $P, Q$ and $R$ using eq. (114), as well as eqs (47)-(49) where we replace $U, V$ and $W$ by $P, Q$ and $R$, respectively. The three other independent covariant components of $\delta \boldsymbol{t}$, i.e. $\delta t_{\chi \chi}, \delta t_{v v}$ and $\delta t_{\chi v}$, are expressed in terms of $U, V$ and $W$ using eqs (113), (50), (63), (64), and (65).

By equating the $q$-covariant component of the Helmholtz representation of $\mathbf{e}_{q} \cdot \delta \boldsymbol{t}$ with the $q q$-covariant component of the right-hand side of eq. (113), we find

$$
\left(1+\frac{\partial h}{\partial q}\right) P=\lambda_{0}(\nabla \cdot \mathbf{s})\left(1+\frac{\partial h}{\partial q}\right)^{2}+2 \mu_{0} e_{q q} .
$$

The exact expressions for $\boldsymbol{\nabla} \cdot \mathbf{s}$ and $e_{q q}$ have been given in Section 3. By taking the Fourier transform of eq. (115), expanding the Fourier transforms of $U, V$ and $P$ in surface spherical harmonics, limiting the development of $h$ to first order, and using formulae provided in Appendix A5, we obtain

$$
\begin{aligned}
\beta_{0} \frac{d U_{\ell}^{m}}{d q}= & P_{\ell}^{m}-\frac{\lambda_{0}}{q}\left[2 U_{\ell}^{m}-\ell(\ell+1) V_{\ell}^{m}\right]+\sum_{\ell^{\prime}=|\ell-2|}^{\ell+2}\left[\begin{array}{ccc}
\ell & 2 & \ell^{\prime} \\
0 & 0 & 0 \\
m & 0 & m
\end{array}\right] \frac{\lambda_{0}}{q}\left[\left(\frac{d h_{2}}{d q}-\frac{h_{2}}{q}\right)\left\{\begin{array}{c}
2 U_{\ell^{\prime}}^{m}-\ell^{\prime}\left(\ell^{\prime}+1\right) V_{\ell^{\prime}}^{m} \\
0
\end{array}\right\}+6 h_{2}\left\{\begin{array}{c}
\frac{d V_{\ell^{\prime}}^{m}}{d q} \\
0
\end{array}\right\}\right] \\
& -\sum_{\ell^{\prime}=|\ell-2|}^{\ell+2}\left[\begin{array}{ccc}
\ell & 2 & \ell^{\prime} \\
0 & 1 & -1 \\
m & 0 & m
\end{array}\right] 4 \sqrt{3} L_{0}^{\ell^{\prime}} \frac{\lambda_{0}}{q} h_{2}\left\{\begin{array}{c}
\frac{d V_{\ell^{\prime}}^{m}}{d q} \\
0
\end{array}\right\},
\end{aligned}
$$

where

$\beta_{0}=\lambda_{0}+2 \mu_{0}$.

The sums over $\ell^{\prime}$ stem from products of spherical harmonics. Their terms are of first order in $h_{2}$. In these terms, the first and second derivatives with respect to $q$ of the harmonic components need to be computed only to zeroth order in $h_{2}$. This can be done analytically once all the scalar equations of motion are written. Arrays with two lines in curly brackets, such as

$\left\{\begin{array}{c}\frac{d V_{\ell^{\prime}}^{m}}{d q} \\ 0\end{array}\right\}$

mean that the upper line must be chosen if $\left|\ell-\ell^{\prime}\right|$ is even or zero and the lower line must be chosen if $\left|\ell-\ell^{\prime}\right|$ is odd. Symbols like

$\left[\begin{array}{ccc}\ell & 2 & \ell^{\prime} \\ 0 & 0 & 0 \\ m & 0 & m\end{array}\right]$ 
are numbers called J-squares by Smith (1974). They arise from products of spherical harmonics and involve products of Wigner 3-j symbols (Edmonds 1960, see Appendix A3).

In order to obtain similar equations for the radial derivatives of $V^{m}{ }_{\ell}$ and $W^{m}{ }_{\ell}$, we form the combinations

$\frac{1}{\sin \chi}\left\{\frac{\partial}{\partial \chi}\left[\frac{\sin \chi \delta t_{q \chi}}{q+h}\right]+\frac{\partial}{\partial v}\left[\frac{\delta t_{q v}}{(q+h) \sin \chi}\right]\right\}$

and

$\frac{1}{\sin \chi}\left\{\frac{\partial}{\partial v}\left(\frac{\delta t_{q \chi}}{q+h}\right)-\frac{\partial}{\partial \chi}\left(\frac{\delta t_{q v}}{q+h}\right)\right\}$.

To first order in $h$, they give, respectively,

$$
\begin{aligned}
& \mu_{0} \ell(\ell+1) \frac{d V_{\ell}^{m}}{d q}=\ell(\ell+1)\left[Q_{\ell}^{m}-\frac{\mu_{0}}{q}\left(U_{\ell}^{m}-V_{\ell}^{m}\right)\right]
\end{aligned}
$$

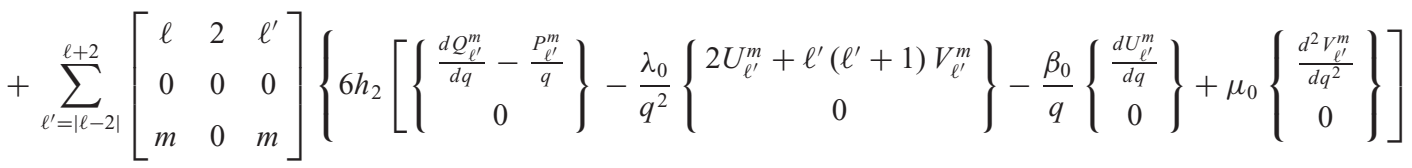

$$
\begin{aligned}
& \left.+\mu_{0}\left(\frac{d h_{2}}{d q}-\frac{h_{2}}{q}\right)\left\{\begin{array}{c}
\ell^{\prime}\left(\ell^{\prime}+1\right)\left(\frac{U_{\ell^{\prime}}^{m}}{q}-\frac{V_{\ell^{\prime}}^{m}}{q}\right)+6 \frac{d V_{\ell^{\prime}}^{m}}{d q} \\
0
\end{array}\right\}\right\} \\
& +\sum_{\ell^{\prime}=|\ell-2|}^{\ell+2}\left[\begin{array}{ccc}
\ell & 2 & \ell^{\prime} \\
0 & 1 & -1 \\
m & 0 & m
\end{array}\right] 2 \sqrt{3} L_{0}^{\ell^{\prime}}\left\{h_{2}\left[\left\{\begin{array}{c}
\frac{P_{\ell^{\prime}}^{m}}{q}-\frac{d Q_{\ell^{\prime}}^{m}}{d q} \\
-i \frac{d R_{\ell^{\prime}}^{m}}{d q}
\end{array}\right\}-\frac{\lambda_{0}}{q^{2}}\left\{\begin{array}{c}
2 U_{\ell^{\prime}}^{m}+\ell^{\prime}\left(\ell^{\prime}+1\right) V_{\ell^{\prime}}^{m} \\
0
\end{array}\right\}-\frac{\beta_{0}}{q}\left\{\begin{array}{c}
\frac{d U_{\ell^{\prime}}^{m}}{d q} \\
0
\end{array}\right\}+\mu_{0}\left\{\begin{array}{c}
\frac{d^{2} V_{\ell^{\prime}}^{m}}{d q^{2}} \\
i \frac{d^{2} W_{\ell^{\prime}}^{m}}{d q^{2}}
\end{array}\right\}\right]\right. \\
& \left.-\mu_{0}\left(\frac{d h_{2}}{d q}-\frac{h_{2}}{q}\right)\left\{\begin{array}{l}
\frac{U_{\ell^{\prime}}^{m}}{q}-\frac{V_{\ell^{\prime}}^{m}}{q}-\frac{d V_{\ell^{\prime}}^{m}}{d q} \\
i\left(\frac{W_{\ell^{\prime}}^{m}}{q}-\frac{d W_{\ell^{\prime}}^{m}}{d q}\right)
\end{array}\right\}\right\}
\end{aligned}
$$

and

$$
\begin{aligned}
& \mu_{0} \ell(\ell+1) \frac{d W_{\ell}^{m}}{d q}=\ell(\ell+1)\left(R_{\ell}^{m}+\mu_{0} \frac{W_{\ell}^{m}}{q}\right) \\
& +\sum_{\ell^{\prime}=|\ell-2|}^{\ell+2}\left[\begin{array}{ccc}
\ell & 2 & \ell^{\prime} \\
0 & 0 & 0 \\
m & 0 & m
\end{array}\right]\left\{6 h_{2}\left[\left\{\begin{array}{c}
-\frac{d R_{\ell^{\prime}}^{m}}{d q} \\
0
\end{array}\right\}+\mu_{0}\left\{\begin{array}{c}
\frac{d^{2} W_{\ell^{\prime}}^{m}}{d q^{2}} \\
0
\end{array}\right\}\right]+\mu_{0}\left(\frac{d h_{2}}{d q}-\frac{h_{2}}{q}\right)\left\{\begin{array}{c}
\left.6 \frac{d W_{\ell^{\prime}}^{m}}{d q}-\ell^{\prime}\left(\ell^{\prime}+1\right) \frac{W_{\ell^{\prime}}^{m}}{q}\right\} \\
0
\end{array}\right\}\right. \\
& +\sum_{\ell^{\prime}=|\ell-2|}^{\ell+2}\left[\begin{array}{ccc}
\ell & 2 & \ell^{\prime} \\
0 & 1 & -1 \\
m & 0 & m
\end{array}\right] 2 \sqrt{3} L_{0}^{\ell^{\prime}}\left\{h_{2}\left[\left\{\begin{array}{c}
-\frac{d R_{\ell^{\prime}}^{m}}{d q} \\
-i \frac{P_{\ell^{\prime}}^{m}}{q}
\end{array}\right\}+\frac{\lambda_{0}}{q^{2}}\left\{i\left[2 U_{\ell^{\prime}}^{m}+\ell^{\prime}\left(\ell^{\prime}+1\right) V_{\ell^{\prime}}^{m}\right]\right\}\right]\right. \\
& \left.+h_{2} \frac{\beta_{0}}{q}\left\{\begin{array}{c}
0 \\
i \frac{d U_{\ell^{\prime}}^{m}}{d q}
\end{array}\right\}+\mu_{0}\left[h_{2}\left\{\begin{array}{c}
\frac{d^{2} W_{\ell^{\prime}}^{m}}{d q^{2}} \\
-i \frac{d^{2} V_{\ell^{\prime}}^{m}}{d q^{2}}
\end{array}\right\}+\left(\frac{d h_{2}}{d q}-\frac{W_{2}}{q}\right)\left\{i\left(-\frac{U_{\ell^{\prime}}^{m}}{q}+\frac{d W_{\ell^{\prime}}^{m}}{d q}+\frac{V_{\ell^{\prime}}^{m}}{q}-\frac{d V_{\ell^{\prime}}^{m}}{d q}\right)\right\}\right]\right\} .
\end{aligned}
$$

\subsection{Poisson's equation}

If we define $\Delta g$ by

$$
\Delta g=\mathbf{n} \cdot[\nabla(\Delta \phi)+4 \pi \mathrm{G} \rho \mathbf{s}],
$$

where $\mathbf{n}$ is the unit normal to a surface $q=$ const and is given by eq. (8), we have to the second order

$$
\begin{aligned}
\frac{\partial}{\partial q} \Delta \phi= & {\left[1+\frac{\partial h}{\partial q}-2 \frac{h^{2}}{q^{2}}-\frac{1}{2 q^{2}}\left(\frac{\partial h}{\partial \chi}\right)^{2}\right] \Delta g+\frac{1}{q^{2}} \frac{\partial h}{\partial \chi}\left(1+\frac{\partial h}{\partial q}-\frac{2 h}{q}\right) \frac{\partial}{\partial \chi} \Delta \phi } \\
& +4 \pi \mathrm{G} \rho_{0}\left\{-\left[1+\frac{\partial h}{\partial q}-\frac{1}{q^{2}}\left(\frac{\partial h}{\partial \chi}\right)^{2}\right] U+\frac{1}{q} \frac{\partial h}{\partial \chi}\left(1+\frac{\partial h}{\partial q}-\frac{h}{q}\right)\left(\frac{\partial V}{\partial \chi}+\frac{1}{\sin \chi} \frac{\partial W}{\partial v}\right)-\frac{1}{q}\left(\frac{\partial h}{\partial \chi}\right)^{2} \frac{\partial V}{\partial q}\right\} .
\end{aligned}
$$


Taking the Fourier transform of this equation, expanding the Fourier transforms of $\Delta g, \Delta \phi$, and $U$ in surface spherical harmonics, and denoting their harmonic components by $g^{m}{ }_{\ell}, \phi^{m}{ }_{\ell}$, and $U_{\ell}^{m}$, respectively, we obtain

$$
\begin{aligned}
& \frac{d \phi_{\ell}^{m}}{d q}=\left(1+\frac{d h_{0}}{d q}-\frac{h_{2}^{2}}{q^{2}}\right) g_{\ell}^{m}-4 \pi \mathrm{G} \rho_{0}\left[\left(1+\frac{d h_{0}}{d q}-\frac{6}{5} \frac{h_{2}^{2}}{q^{2}}\right) U_{\ell}^{m}+\frac{6}{5} \frac{h_{2}^{2}}{q} \frac{d V_{\ell}^{m}}{d q}\right] \\
& +\sum_{\ell^{\prime}=|\ell-2|}^{\ell+2}\left[\begin{array}{ccc}
\ell & 2 & \ell^{\prime} \\
0 & 0 & 0 \\
m & 0 & m
\end{array}\right]\left\{\left(\frac{d h_{2}}{d q}-\frac{5}{7} \frac{h_{2}^{2}}{q^{2}}\right)\left\{\begin{array}{c}
g_{\ell^{\prime}}^{m} \\
0
\end{array}\right\}-4 \pi \mathrm{G} \rho_{0}\left[\left(\frac{d h_{2}}{d q}-\frac{2}{7} \frac{h_{2}^{2}}{q^{2}}\right)\left\{\begin{array}{c}
U_{\ell^{\prime}}^{m} \\
0
\end{array}\right\}+\frac{2}{7} \frac{h_{2}^{2}}{q}\left\{\begin{array}{c}
\frac{d V_{\ell^{\prime}}^{m}}{d q} \\
0
\end{array}\right\}\right]\right\} \\
& +\sum_{\ell^{\prime}=|\ell-4|}^{\ell+4}\left[\begin{array}{ccc}
\ell & 4 & \ell^{\prime} \\
0 & 0 & 0 \\
m & 0 & m
\end{array}\right]\left\{\frac{d h_{4}}{d q}\left\{\begin{array}{c}
g_{\ell^{\prime}}^{m} \\
0
\end{array}\right\}-4 \pi \mathrm{G} \rho_{0}\left[\left(\frac{d h_{4}}{d q}+\frac{72}{35} \frac{h_{2}^{2}}{q^{2}}\right)\left\{\begin{array}{c}
U_{\ell^{\prime}}^{m} \\
0
\end{array}\right\}-\frac{72}{35} \frac{h_{2}^{2}}{q}\left\{\begin{array}{c}
\frac{d V_{\ell^{\prime}}^{m}}{d q} \\
0
\end{array}\right\}\right]\right\} \\
& +\sum_{\ell^{\prime}=|\ell-2|}^{\ell+2}\left[\begin{array}{ccc}
\ell & 2 & \ell^{\prime} \\
0 & 1 & -1 \\
m & 0 & m
\end{array}\right] 2 \sqrt{3} L_{0}^{\ell^{\prime}} \frac{h_{2}}{q}\left\{\begin{array}{c}
-\frac{\phi_{\ell^{\prime}}^{m}}{q}-4 \pi \mathrm{G} \rho_{0} V_{\ell^{\prime}}^{m} \\
4 \pi \mathrm{G} \rho_{0} i W_{\ell^{\prime}}^{m}
\end{array}\right\} \\
& +\sum_{\ell^{\prime}=|\ell-4|}^{\ell+4}\left[\begin{array}{ccc}
\ell & 4 & \ell^{\prime} \\
0 & 1 & -1 \\
m & 0 & m
\end{array}\right] 2 \sqrt{10} L_{0}^{\ell^{\prime}} \frac{h_{4}}{q}\left\{\begin{array}{c}
-\frac{\phi_{\ell^{\prime}}^{m}}{q}-4 \pi \mathrm{G} \rho_{0} V_{\ell^{\prime}}^{m} \\
4 \pi \mathrm{G} \rho_{0} i W_{\ell^{\prime}}^{m}
\end{array}\right\} \\
& +\sum_{\ell^{\prime}=|\ell-2| \ell^{\prime \prime}=\left|\ell^{\prime}-2\right|}^{\ell+2} \sum_{\ell^{\prime}+2}\left[\begin{array}{ccc}
\ell & 2 & \ell^{\prime} \\
0 & 0 & 0 \\
m & 0 & m
\end{array}\right]\left[\begin{array}{ccc}
\ell^{\prime} & 2 & \ell^{\prime \prime} \\
0 & 1 & -1 \\
m & 0 & m
\end{array}\right] 2 \sqrt{3} L_{0}^{\ell^{\prime \prime}} \frac{h_{2}}{q}\left[\left(\frac{d h_{2}}{d q}-2 \frac{h_{2}}{q}\right)\left\{\begin{array}{c}
-\frac{\phi_{\ell^{\prime \prime}}^{m}}{q} \\
0
\end{array}\right\}+4 \pi \mathrm{G} \rho_{0}\left(\frac{d h_{2}}{d q}-\frac{h_{2}}{q}\right)\left\{\begin{array}{l}
-V_{\ell^{\prime \prime}}^{m} \\
i W_{\ell^{\prime \prime}}^{m}
\end{array}\right\}\right]
\end{aligned}
$$

In order to derive eq. (122), we have used formulae provided in Appendices A4 and A5. Poisson's equation (95) then gives

$$
\begin{aligned}
\frac{d g_{\ell}^{m}}{d q}= & \left\{\ell(\ell+1)\left[1-2 \frac{h_{0}}{q}+\frac{d h_{0}}{d q}-\frac{2}{5} \frac{h_{2}}{q}\left(2 \frac{h_{2}}{q}+\frac{d h_{2}}{d q}\right)\right]+3 m^{2} \frac{h_{2}^{2}}{q^{2}}\right\} \frac{\phi_{\ell}^{m}}{q^{2}} \\
& +\left[-2+2 \frac{h_{0}}{q}-2 \frac{d h_{0}}{d q}+\frac{2}{5} \frac{h_{2}}{q}\left(\frac{h_{2}}{q}+\frac{d h_{2}}{d q}\right)\right] \frac{g_{\ell}^{m}}{q} \\
& +4 \pi \mathrm{G}\left\{\left[\ell(\ell+1)\left(1+\frac{d h_{0}}{d q}-\frac{h_{0}}{q}-\frac{6}{5} \frac{h_{2}^{2}}{q^{2}}-\frac{1}{5} \frac{h_{2}}{q} \frac{d h_{2}}{d q}\right)+3 m^{2} \frac{h_{2}^{2}}{q^{2}}\right] \frac{\rho_{0}}{q} V_{\ell}^{m}\right. \\
& \left.+\frac{2}{5}\left(\frac{d h_{2}}{d q}\right)^{2}\left[\frac{d}{d q}\left(\rho_{0} U_{\ell}^{m}\right)+\frac{\rho_{0}}{q} U_{\ell}^{m}\right]-\frac{12}{5} \frac{h_{2}^{2}}{q^{2}} \frac{\rho_{0}}{q} U_{\ell}^{m}\right\} \\
& +\sum_{\ell^{\prime}=\ell \ell-2 \mid}^{\ell+2}\left[\begin{array}{ccc}
\ell & 2 & \ell^{\prime} \\
0 & 0 & 0 \\
m & 0 & m
\end{array}\right]\left\{\left\{\frac{\ell^{\prime}\left(\ell^{\prime}+1\right)}{q^{2}}\left[\frac{d h_{2}}{d q}-2 \frac{h_{2}}{q}+\frac{h_{2}}{q}\left(\frac{h_{2}}{q}-\frac{4}{7} \frac{d h_{2}}{d q}\right)\right]+6 m^{2} \frac{h_{2}^{2}}{q^{4}}\right\}\left\{\begin{array}{c}
\phi_{\ell^{\prime}}^{m} \\
0
\end{array}\right\}\right. \\
& -\frac{2}{q}\left[\frac{d h_{2}}{d q}+2 \frac{h_{2}}{q}-\frac{2}{7} \frac{h_{2}}{q}\left(\frac{h_{2}}{q}+\frac{d h_{2}}{d q}\right)\right]\left\{\begin{array}{c}
g_{\ell^{\prime}}^{m} \\
0
\end{array}\right\} \\
& +4 \pi \mathrm{G}\left\{\frac{4}{7}\left(\frac{d h_{2}}{d q}\right)^{2}\left\{\begin{array}{c}
\frac{d}{d q}\left(\rho_{0} U_{\ell^{\prime}}^{m}\right) \\
0
\end{array}\right\}+\frac{\rho_{0}}{q}\left\{6 \frac{h_{2}}{q}+\frac{2}{7}\left[2\left(\frac{d h_{2}}{d q}\right)^{2}+5 \frac{h_{2}}{q} \frac{d h_{2}}{d q}-12 \frac{h_{2}^{2}}{q^{2}}\right]\right\}\left\{\begin{array}{c}
U_{\ell^{\prime}}^{m} \\
0
\end{array}\right\}\right. \\
& \left.\left.+\rho_{0} \frac{h_{2}}{q}\left(6-\frac{10}{7} \frac{h_{2}}{q}\right)\left\{\frac{d}{d q} V_{\ell^{\prime}}^{m}\right\}+\frac{\rho_{0}}{q}\left[\ell^{\prime}\left(\ell^{\prime}+1\right)\left(\frac{d h_{2}}{d q}-\frac{h_{2}}{q}+\frac{3}{7} \frac{h_{2}^{2}}{q^{2}}-\frac{2}{7} \frac{h_{2}}{q} \frac{d h_{2}}{d q}\right)+6 m^{2} \frac{h_{2}^{2}}{q^{2}}\right]\left\{\begin{array}{c}
V_{\ell^{\prime}}^{m} \\
0
\end{array}\right\}\right\}\right\}
\end{aligned}
$$




$$
\begin{aligned}
& +\sum_{\ell^{\prime}=|\ell-4|}^{\ell+4}\left[\begin{array}{ccc}
\ell & 4 & \ell^{\prime} \\
0 & 0 & 0 \\
m & 0 & m
\end{array}\right]\left\{\frac{\ell^{\prime}\left(\ell^{\prime}+1\right)}{q^{2}}\left[\frac{d h_{4}}{d q}-2 \frac{h_{4}}{q}+\frac{18}{35} \frac{h_{2}}{q}\left(11 \frac{h_{2}}{q}-2 \frac{d h_{2}}{d q}\right)\right]\left\{\begin{array}{c}
\phi_{\ell^{\prime}}^{m} \\
0
\end{array}\right\}\right. \\
& -\frac{2}{q}\left[\frac{d h_{4}}{d q}+9 \frac{h_{4}}{q}-\frac{36}{35} \frac{h_{2}}{q}\left(\frac{h_{2}}{q}+\frac{d h_{2}}{d q}\right)\right]\left\{\begin{array}{c}
g_{\ell^{\prime}}^{m} \\
0
\end{array}\right\} \\
& +4 \pi \mathrm{G}\left\{\frac{36}{35}\left(\frac{d h_{2}}{d q}\right)^{2}\left\{\begin{array}{c}
\frac{d}{d q}\left(\rho_{0} U_{\ell^{\prime}}^{m}\right) \\
0
\end{array}\right\}+\frac{\rho_{0}}{q}\left\{20 \frac{h_{4}}{q}+\frac{36}{35}\left[\left(\frac{d h_{2}}{d q}\right)^{2}+5 \frac{h_{2}}{q} \frac{d h_{2}}{d q}-6 \frac{h_{2}^{2}}{q^{2}}\right]\right\}\left\{\begin{array}{c}
U_{\ell^{\prime}}^{m} \\
0
\end{array}\right\}\right. \\
& \left.\left.+\rho_{0}\left(20 \frac{h_{4}}{q}-\frac{180}{35} \frac{h_{2}^{2}}{q^{2}}\right)\left\{\begin{array}{c}
\frac{d}{d q} V_{\ell^{\prime}}^{m} \\
0
\end{array}\right\}+\frac{\rho_{0}}{q} \ell^{\prime}\left(\ell^{\prime}+1\right)\left(\frac{d h_{4}}{d q}-\frac{h_{4}}{q}+\frac{162}{35} \frac{h_{2}^{2}}{q^{2}}-\frac{18}{35} \frac{h_{2}}{q} \frac{d h_{2}}{d q}\right)\left\{\begin{array}{c}
V_{\ell^{\prime}}^{m} \\
0
\end{array}\right\}\right\}\right\} \\
& +\sum_{\ell^{\prime}=|\ell-2|}^{\ell+2}\left[\begin{array}{ccc}
\ell & 2 & \ell^{\prime} \\
0 & 1 & -1 \\
m & 0 & m
\end{array}\right] 2 \sqrt{3} L_{0}^{\ell^{\prime}}\left[\frac{h_{2}}{q}\left\{\begin{array}{c}
-\frac{g_{\ell^{\prime}}^{m}}{q}+4 \pi \mathrm{G} \rho_{0}\left(\frac{U_{\ell^{\prime}}^{m}}{q}-\frac{d V_{\ell^{\prime}}^{m}}{d q}\right) \\
-4 \pi \mathrm{G} \rho_{0} i \frac{d W_{\ell^{\prime}}^{m}}{d q}
\end{array}\right\}\right. \\
& \left.+\left(\frac{d h_{2}}{d q}-2 \frac{h_{2}}{q}-2 \frac{h_{2}^{2}}{q^{2}}\right)\left\{\begin{array}{c}
\frac{\phi_{\ell^{\prime}}^{m}}{q^{2}} \\
0
\end{array}\right\}+4 \pi \mathrm{G} \frac{\rho_{0}}{q}\left(-\frac{d h_{2}}{d q}+\frac{h_{2}^{2}}{q^{2}}\right)\left\{\begin{array}{c}
-V_{\ell^{\prime}}^{m} \\
i W_{\ell^{\prime}}^{m}
\end{array}\right\}\right] \\
& +\sum_{\ell^{\prime}=|\ell-4|}^{\ell+4}\left[\begin{array}{ccc}
\ell & 4 & \ell^{\prime} \\
0 & 1 & -1 \\
m & 0 & m
\end{array}\right] 2 \sqrt{10} L_{0}^{\ell^{\prime}}\left[\frac{h_{4}}{q}\left\{\begin{array}{c}
-\frac{g_{\ell^{\prime}}^{m}}{q}+4 \pi \mathrm{G} \rho_{0}\left(\frac{U_{\ell^{\prime}}^{m}}{q}-\frac{d V_{\ell^{\prime}}^{m}}{d q}\right) \\
-4 \pi \mathrm{G} \rho_{0} i \frac{d W_{\ell^{\prime}}^{m}}{d q}
\end{array}\right\}\right. \\
& \left.+\left(\frac{d h_{4}}{d q}-2 \frac{h_{4}}{q}\right)\left\{\begin{array}{c}
\frac{\phi_{\ell^{\prime}}^{m}}{q^{2}} \\
0
\end{array}\right\}+4 \pi \mathrm{G} \frac{\rho_{0}}{q}\left(-\frac{d h_{4}}{d q}-\frac{h_{4}}{q}\right)\left\{\begin{array}{c}
-V_{\ell^{\prime}}^{m} \\
i W_{\ell^{\prime}}^{m}
\end{array}\right\}\right] \\
& +\sum_{\ell^{\prime}=|\ell-2|}^{\ell+2} \sum_{\ell^{\prime \prime}=\left|\ell^{\prime}-2\right|}^{\ell^{\prime}+2}\left[\begin{array}{ccc}
\ell & 2 & \ell^{\prime} \\
0 & 0 & 0 \\
m & 0 & m
\end{array}\right]\left[\begin{array}{ccc}
\ell^{\prime} & 2 & \ell^{\prime \prime} \\
0 & 1 & -1 \\
m & 0 & m
\end{array}\right] 2 \sqrt{3} L_{0}^{\ell^{\prime \prime}}\left\{12 \frac{h_{2}^{2}}{q^{2}}\left\{\begin{array}{c}
-\frac{\phi_{\ell^{\prime \prime}}^{m}}{q^{2}} \\
0
\end{array}\right\}+\frac{h_{2}}{q}\left(1-2 \frac{h_{2}}{q}+\frac{d h_{2}}{d q}\right)\left\{\begin{array}{c}
-\frac{g_{\ell^{\prime \prime}}^{m}}{q} \\
0
\end{array}\right\}\right. \\
& \left.+4 \pi \mathrm{G} \frac{\rho_{0}}{q} \frac{h_{2}}{q}\left[\left(\frac{d h_{2}}{d q}-2 \frac{h_{2}}{q}\right)\left\{\begin{array}{c}
U_{\ell^{\prime \prime}}^{m} \\
0
\end{array}\right\}-2\left(\frac{d h_{2}}{d q}+4 \frac{h_{2}}{q}\right)\left\{\begin{array}{c}
-V_{\ell^{\prime \prime}}^{m} \\
i W_{\ell^{\prime \prime}}^{m}
\end{array}\right\}+h_{2}\left\{\begin{array}{c}
-\frac{V_{\ell^{\prime \prime}}^{m}}{q}+\frac{d V_{\ell^{\prime \prime}}^{m}}{d q} \\
2 i \frac{W_{\ell^{\prime \prime}}^{m}}{q}+i \frac{d W_{\ell^{\prime \prime}}^{m}}{d q}
\end{array}\right\}\right]\right\} \\
& +\sum_{\ell^{\prime}=|\ell-2| \sum^{\prime \prime}=\left|\ell^{\prime}-2\right|}^{\ell+2}\left[\begin{array}{ccc}
\ell & 2 & \ell^{\prime} \\
0 & 1 & -1 \\
m & 0 & m
\end{array}\right]\left[\begin{array}{ccc}
\ell^{\prime} & 2 & \ell^{\prime \prime} \\
0 & 1 & -1 \\
m & 0 & m
\end{array}\right] 12 L_{0}^{\ell^{\prime}} L_{0}^{\ell^{\prime \prime}} 4 \pi \mathrm{G} \frac{\rho_{0}}{q} \frac{h_{2}^{2}}{q^{2}}\left\{\begin{array}{c}
0 \\
i W_{\ell^{\prime \prime}}^{m}
\end{array}\right\} \text {. }
\end{aligned}
$$

In accordance with the conclusions of Section 6 , we have extended the computation of the terms containing $4 \pi \mathrm{G} \rho_{0}$ to the second order in $h$. The derivatives of the harmonic components appearing in the sums over $\ell^{\prime}$ must be computed to the first order in $h$ when they are multiplied by a coefficient of the first order in $h$. Otherwise, when they are multiplied by a coefficient of the second order in $h$, they need to be computed only to the zeroth order in $h$.

\subsection{Conservation of linear momentum}

The Fourier transform of the equation of motion (94) can be written

$\nabla \cdot \delta \boldsymbol{t}=\rho\left(\boldsymbol{\eta}_{\mathbf{1}}+\boldsymbol{\eta}_{\mathbf{2}}\right)$,

where

$\boldsymbol{\eta}_{\mathbf{1}}=-\omega^{2} \mathbf{s}+2 i \omega \boldsymbol{\Omega}_{0} \times \mathbf{s}$

and

$\eta_{2}=\nabla(\Delta \phi)+\nabla(\mathbf{s} \cdot \nabla \tilde{\phi})-(\nabla \cdot \mathbf{s}) \nabla \tilde{\phi}$. 
In order to avoid cumbersome notation, we have not underlined the Fourier transforms of $\delta \boldsymbol{t}, \mathbf{s}$ and $\Delta \phi$. The context being clear, no confusion should arise from using the same notation for two different functions. We showed in Section 6 that, in coordinates $(q, \chi, v), \nabla \cdot \delta \boldsymbol{t}$ and $\boldsymbol{\eta}_{\boldsymbol{l}}$ have to be computed to first order in $h$ and $\eta_{2}$ has to be computed to second order in $h$.

The contravariant components of $\boldsymbol{\nabla} \cdot \delta \boldsymbol{t}$ can be computed from eqs (73-75), which involve the contravariant components of $\delta \boldsymbol{t}$ that are obtained by index-raising twice the covariant components of $\delta \boldsymbol{t}$. Components $\delta t_{q q}, \delta t_{q \chi}$ and $\delta t_{q v}$ are expressed in terms of $P, Q$ and $R$, whereas components $\delta t_{\chi \chi}, \delta t_{\chi v}$ and $\delta t_{v v}$ are expressed in terms of $U, V$ and $W$.

Consequently, the $q$-contravariant component of the equation of motion gives for the radial derivative of $P^{m}{ }_{\ell}$ :

$$
\begin{aligned}
& \frac{d P_{\ell}^{m}}{d q}=\rho_{0}\left(\eta_{1 \ell}^{U^{m}}+\eta_{2 \ell}^{U^{m}}\right)-2 \frac{P_{\ell}^{m}}{q}+\ell(\ell+1) \frac{Q_{\ell}^{m}}{q}-\frac{h_{2}}{q} \frac{d Q_{\ell}^{m}}{d q}+\frac{2}{q^{2}}\left(\lambda_{0}+\mu_{0}\right)\left[2 U_{\ell}^{m}-\ell(\ell+1) V_{\ell}^{m}\right]+\frac{2 \lambda_{0}}{q} \frac{d U_{\ell}^{m}}{d q} \\
& -\sum_{\ell^{\prime}=|\ell-2|}^{\ell+2}\left[\begin{array}{ccc}
\ell & 2 & \ell^{\prime} \\
0 & 0 & 0 \\
m & 0 & m
\end{array}\right]\left\{\left(q \frac{d^{2} h_{2}}{d q^{2}}-4 \frac{d h_{2}}{d q}-2 \frac{h_{2}}{q}\right)\left\{\begin{array}{c}
\frac{P_{\ell^{\prime}}^{m}}{q} \\
0
\end{array}\right\}-3 \frac{d h_{2}}{d q}\left\{\begin{array}{c}
\frac{d P_{\ell^{\prime}}^{m}}{d q} \\
0
\end{array}\right\}\right. \\
& +\left(\frac{h_{2}}{q}+2 \frac{d h_{2}}{d q}\right) \ell^{\prime}\left(\ell^{\prime}+1\right)\left\{\begin{array}{c}
\frac{Q_{\ell^{\prime}}^{m}}{q} \\
0
\end{array}\right\}+2 \frac{h_{2}}{q}\left\{\begin{array}{c}
\frac{d Q_{\ell^{\prime}}^{m}}{d q} \\
0
\end{array}\right\} \\
& +\left(2 \frac{h_{2}}{q}+\frac{d h_{2}}{d q}\right) \frac{2}{q^{2}}\left(\lambda_{0}+\mu_{0}\right)\left\{\begin{array}{c}
2 U_{\ell^{\prime}}^{m}-\ell^{\prime}\left(\ell^{\prime}+1\right) V_{\ell^{\prime}}^{m} \\
0
\end{array}\right\} \\
& \left.+\frac{\lambda_{0}}{q}\left(2 \frac{h_{2}}{q}+3 \frac{d h_{2}}{d q}\right)\left\{\begin{array}{c}
\frac{d U_{\ell^{\prime}}^{m}}{d q} \\
0
\end{array}\right\}-\frac{12 h_{2}}{q^{2}}\left(\lambda_{0}+\mu_{0}\right)\left\{\begin{array}{c}
\frac{d V_{\ell^{\prime}}^{m}}{d q} \\
0
\end{array}\right\}\right\} \\
& -\sum_{\ell^{\prime}=|\ell-2|}^{\ell+2}\left[\begin{array}{ccc}
\ell & 2 & \ell^{\prime} \\
0 & 1 & -1 \\
m & 0 & m
\end{array}\right] 2 \sqrt{3} L_{0}^{\ell^{\prime}}\left\{\left(3 \frac{h_{2}}{q}+\frac{d h_{2}}{d q}\right)\left\{\begin{array}{c}
\frac{Q_{\ell^{\prime}}^{m}}{q} \\
-i \frac{R_{\ell^{\prime}}^{m}}{q}
\end{array}\right\}+\frac{h_{2}}{q}\left\{\begin{array}{c}
-2 \frac{d Q_{\ell^{\prime}}^{m}}{d q} \\
3 i \frac{d R_{\ell^{\prime}}^{m}}{d q}
\end{array}\right\}\right. \\
& +\frac{h_{2}}{q^{3}} \beta_{0}\left\{\begin{array}{c}
2 U_{\ell^{\prime}}^{m}-\ell^{\prime}\left(\ell^{\prime}+1\right) V_{\ell^{\prime}}^{m} \\
0
\end{array}\right\}+\frac{h_{2}}{q^{2}} \lambda_{0}\left\{\begin{array}{c}
\frac{d U_{\ell^{\prime}}^{m}}{d q}-4 \frac{d V_{\ell^{\prime}}^{m}}{d q} \\
0
\end{array}\right\}+\frac{h_{2}}{q^{2}} \mu_{0}\left\{i\left[\ell^{\prime}\left(\ell^{\prime}+1\right) \frac{-2 \frac{d V_{\ell^{\prime}}^{m}}{d q}}{q}-2 \frac{W_{\ell^{\prime}}^{m}}{d q}\right]\right\},
\end{aligned}
$$

where

$$
\begin{aligned}
\eta_{1 \ell}^{U^{m}}= & -\omega^{2}\left\{U_{\ell}^{m}+\sum_{\ell^{\prime}=|\ell-2|}^{\ell+2}\left[\left[\begin{array}{ccc}
\ell & 2 & \ell^{\prime} \\
0 & 0 & 0 \\
m & 0 & m
\end{array}\right] \frac{d h_{2}}{d q}\left\{\begin{array}{c}
-U_{\ell^{\prime}}^{m} \\
0
\end{array}\right\}+\left[\begin{array}{ccc}
\ell & 2 & \ell^{\prime} \\
0 & 1 & -1 \\
m & 0 & m
\end{array}\right] 2 \sqrt{3} \frac{h_{2}}{q} L_{0}^{\ell^{\prime}}\left\{\begin{array}{c}
V_{\ell^{\prime}}^{m} \\
-i W_{\ell^{\prime}}^{m}
\end{array}\right\}\right]\right. \\
& +2 \omega \Omega_{0}\left\{\sum_{\ell^{\prime}=|\ell-1|}^{\ell+1}\left[\begin{array}{ccc}
\ell & 1 & \ell^{\prime} \\
0 & 1 & -1 \\
m & 0 & m
\end{array}\right] 2 L_{0}^{\ell^{\prime}}\left(1+\frac{h_{2}}{q}\right)\left\{\begin{array}{c}
-V_{\ell^{\prime} m} \\
i W_{\ell^{\prime} m}
\end{array}\right\}-\frac{6}{5} h_{2}\left[\begin{array}{ccc}
\ell & 1 & \ell^{\prime} \\
0 & 0 & 0 \\
m & 0 & m
\end{array}\right]\left\{\begin{array}{c}
0 \\
i \frac{d W_{\ell^{\prime} m}}{d q}
\end{array}\right\}\right. \\
& +\sum_{\ell^{\prime}=|\ell-3|}^{\ell+3} \frac{6}{5} h_{2}\left[\begin{array}{ccc}
\ell & 3 & \ell^{\prime} \\
0 & 0 & 0 \\
m & 0 & m
\end{array}\right]\left\{\begin{array}{c}
0 \\
\left.i \frac{d W_{\ell^{\prime} m}}{d q}\right\} \\
+
\end{array}\right. \\
& \left.\sum_{\ell^{\prime}=|\ell-2| \ell^{\prime \prime}=\left|\ell^{\prime}-1\right|}^{\ell+2} \sum_{\ell^{\prime}+1}\left[\begin{array}{ccc}
\ell & 2 & \ell^{\prime} \\
0 & 0 & 0 \\
m & 0 & m
\end{array}\right]\left[\begin{array}{ccc}
\ell^{\prime} & 1 & \ell^{\prime \prime} \\
0 & 1 & -1 \\
m & 0 & m
\end{array}\right]\left(2 \frac{h_{2}}{q}-\frac{\partial h_{2}}{\partial q}\right) 2 L_{0}^{\ell^{\prime \prime}}\left\{\begin{array}{l}
-V_{\ell^{\prime \prime} m} \\
i W_{\ell^{\prime \prime} m}
\end{array}\right\}\right\}
\end{aligned}
$$

and

$$
\begin{aligned}
\eta_{2 \ell}^{U^{m}}=[1- & \left.\frac{d h_{0}}{d q}+\frac{1}{5}\left(\frac{d h_{2}}{d q}\right)^{2}\right]\left(g_{\ell}^{m}-4 \pi \mathrm{G} \rho_{0} U_{\ell}^{m}\right)+\frac{1}{5} \frac{h_{2}^{2}}{q^{2}} g_{\ell}^{m}-4 \pi \mathrm{G} \rho_{0} \frac{6}{5} \frac{h_{2}^{2}}{q} \frac{d V_{\ell}^{m}}{d q} \\
& +\sum_{\ell^{\prime}=|\ell-2|}^{\ell+2}\left[\begin{array}{ccc}
\ell & 2 & \ell^{\prime} \\
0 & 0 & 0 \\
m & 0 & m
\end{array}\right]\left\{\left[-\frac{d h_{2}}{d q}+\frac{2}{7}\left(\frac{d h_{2}}{d q}\right)^{2}\right]\left\{\begin{array}{c}
g_{\ell^{\prime}}^{m}-4 \pi \mathrm{G} \rho_{0} U_{\ell^{\prime}}^{m} \\
0
\end{array}\right\}-\frac{1}{7} \frac{h_{2}^{2}}{q}\left\{\begin{array}{c}
3 \frac{g_{\ell^{\prime}}^{m}}{q}+8 \pi \mathrm{G} \rho_{0} \frac{d V_{\ell^{\prime}}^{m}}{d q} \\
0
\end{array}\right\}\right\}
\end{aligned}
$$




$$
\begin{aligned}
& +\sum_{\ell^{\prime}=|\ell-4|}^{\ell+4}\left[\begin{array}{ccc}
\ell & 4 & \ell^{\prime} \\
0 & 0 & 0 \\
m & 0 & m
\end{array}\right]\left\{\left[-\frac{d h_{4}}{d q}+\frac{18}{35}\left(\frac{d h_{2}}{d q}\right)^{2}\right]\left\{\begin{array}{c}
g_{\ell^{\prime}}^{m}-4 \pi \mathrm{G} \rho_{0} U_{\ell^{\prime}}^{m} \\
0
\end{array}\right\}-\frac{72}{35} \frac{h_{2}^{2}}{q}\left\{\begin{array}{c}
\frac{g_{\ell^{\prime}}^{m}}{q}-4 \pi \mathrm{G} \rho_{0} \frac{d V_{\ell^{\prime}}^{m}}{d q} \\
0
\end{array}\right\}\right\} \\
& +4 \pi \mathrm{G} \rho_{0}\left\{\sum_{\ell^{\prime}=|\ell-2|}^{\ell+2}\left[\begin{array}{ccc}
\ell & 2 & \ell^{\prime} \\
0 & 1 & -1 \\
m & 0 & m
\end{array}\right] 2 \sqrt{3} L_{0}^{\ell^{\prime}} \frac{h_{2}}{q}\left\{\begin{array}{c}
-V_{\ell^{\prime}}^{m} \\
i W_{\ell^{\prime}}^{m}
\end{array}\right\}\right. \\
& +\sum_{\ell^{\prime}=|\ell-4|}^{\ell+4}\left[\begin{array}{ccc}
\ell & 4 & \ell^{\prime} \\
0 & 1 & -1 \\
m & 0 & m
\end{array}\right] 2 \sqrt{10} L_{0}^{\ell^{\prime}} \frac{h_{4}}{q}\left\{\begin{array}{c}
-V_{\ell^{\prime}}^{m} \\
i W_{\ell^{\prime}}^{m}
\end{array}\right\} \\
& \left.-\sum_{\ell^{\prime}=|\ell-2|}^{\ell+2} \sum_{\ell^{\prime \prime}=\left|\ell^{\prime}-2\right|}^{\ell^{\prime}+2}\left[\begin{array}{ccc}
\ell & 2 & \ell^{\prime} \\
0 & 0 & 0 \\
m & 0 & m
\end{array}\right]\left[\begin{array}{ccc}
\ell^{\prime} & 2 & \ell^{\prime \prime} \\
0 & 1 & -1 \\
m & 0 & m
\end{array}\right] 2 \sqrt{3} L_{0}^{\ell^{\prime \prime}} \frac{h_{2}}{q}\left(\frac{d h_{2}}{d q}+\frac{h_{2}}{q}\right)\left\{\begin{array}{c}
-V_{\ell^{\prime}}^{m} \\
i W_{\ell^{\prime}}^{m}
\end{array}\right\}\right\} \\
& +\frac{d \tilde{\phi}_{0}}{d q}\left\{\left[-\frac{2}{q}+2 \frac{h_{0}}{q^{2}}+\frac{4}{q} \frac{d h_{0}}{d q}-\frac{d^{2} h_{0}}{d q^{2}}+\frac{2}{5}\left(\frac{h_{2}^{2}}{q^{3}}-\frac{h_{2}}{q^{2}} \frac{d h_{2}}{d q}+\frac{3}{q}\left(\frac{d h_{2}}{d q}\right)^{2}-\frac{d h_{2}}{d q} \frac{d^{2} h_{2}}{d q^{2}}\right)\right] U_{\ell}^{m}\right. \\
& +\left[\frac{d h_{0}}{d q}-\frac{9}{5}\left(\frac{d h_{2}}{d q}\right)^{2}\right] \frac{d U_{\ell}^{m}}{d q}+\left[3 m^{2} \frac{h_{2}^{2}}{q^{3}}+\frac{\ell(\ell+1)}{q}\left[1-\frac{h_{0}}{q}-2 \frac{d h_{0}}{d q}+\frac{1}{5}\left(-5 \frac{h_{2}^{2}}{q^{2}}+2 \frac{h_{2}}{q} \frac{d h_{2}}{d q}+3\left(\frac{d h_{2}}{d q}\right)^{2}\right)\right]\right] V_{\ell}^{m} \\
& +\frac{18 h_{2}}{5 q} \frac{d h_{2}}{d q} \frac{d V_{\ell}^{m}}{d q}-\frac{12 h_{2}^{2}}{5 q} \frac{d^{2} V_{\ell}^{m}}{d q^{2}} \\
& +\sum_{\ell^{\prime}=|\ell-2|}^{\ell+2}\left[\begin{array}{ccc}
\ell & 2 & \ell^{\prime} \\
0 & 0 & 0 \\
m & 0 & m
\end{array}\right]\left\{\left[\frac{2}{q}\left(\frac{h_{2}}{q}+\frac{d h_{2}}{d q}\right)-\frac{d^{2} h_{2}}{d q^{2}}+\frac{2}{7}\left(-2 \frac{h_{2}^{2}}{q^{3}}-3 \frac{h_{2}}{q^{2}} \frac{d h_{2}}{d q}-\frac{6}{q}\left(\frac{d h_{2}}{d q}\right)^{2}+4 \frac{d h_{2}}{d q} \frac{d^{2} h_{2}}{d q^{2}}\right)\right]\left\{\begin{array}{c}
U_{\ell^{\prime}}^{m} \\
0
\end{array}\right\}\right. \\
& +\frac{d h_{2}}{d q}\left(1-\frac{18}{7} \frac{d h_{2}}{d q}\right)\left\{\begin{array}{c}
\frac{d U_{\ell^{\prime}}^{m}}{d q} \\
0
\end{array}\right\}+\left[6 m^{2} \frac{h_{2}^{2}}{q^{3}}-\frac{\ell^{\prime}\left(\ell^{\prime}+1\right)}{q}\left[\frac{h_{2}}{q}+\frac{d h_{2}}{d q}-\frac{2}{7} \frac{d h_{2}}{d q}\left(2 \frac{h_{2}}{q}+3 \frac{d h_{2}}{d q}\right)\right]\right]\left\{\begin{array}{c}
V_{\ell^{\prime}}^{m} \\
0
\end{array}\right\} \\
& \left.+\frac{h_{2}}{q}\left[-6+\frac{2}{7}\left(18 \frac{d h_{2}}{d q}+5 \frac{h_{2}}{q}\right)\right]\left\{\begin{array}{c}
\frac{d V_{\ell^{\prime}}^{m}}{d q} \\
0
\end{array}\right\}-\frac{4 h_{2}^{2}}{7 q}\left\{\begin{array}{c}
\frac{d^{2} V_{\ell^{\prime}}^{m}}{d q^{2}} \\
0
\end{array}\right\}\right\} \\
& +\sum_{\ell^{\prime}=|\ell-4|}^{\ell+4}\left[\begin{array}{ccc}
\ell & 4 & \ell^{\prime} \\
0 & 0 & 0 \\
m & 0 & m
\end{array}\right]\left\{\left[2 \frac{h_{4}}{q^{2}}+\frac{2}{q} \frac{d h_{4}}{d q}-\frac{d^{2} h_{4}}{d q^{2}}-\frac{36}{35 q}\left(\frac{h_{2}^{2}}{q^{2}}+4 \frac{h_{2}}{q} \frac{d h_{2}}{d q}-2 \frac{d h_{2}}{d q} \frac{d^{2} h_{2}}{d q^{2}}+3\left(\frac{d h_{2}}{d q}\right)^{2}\right)\right]\left\{\begin{array}{c}
U_{\ell^{\prime}}^{m} \\
0
\end{array}\right\}\right. \\
& +\left[\frac{d h_{4}}{d q}-\frac{162}{35}\left(\frac{d h_{2}}{d q}\right)^{2}\right]\left\{\begin{array}{c}
\frac{d U_{\ell^{\prime}}^{m}}{d q} \\
0
\end{array}\right\} \\
& +\left[-\frac{h_{4}}{q^{2}}-\frac{1}{q} \frac{d h_{4}}{d q}+\frac{18}{35 q}\left(5 \frac{h_{2}^{2}}{q^{2}}+2 \frac{h_{2}}{q} \frac{d h_{2}}{d q}+3\left(\frac{d h_{2}}{d q}\right)^{2}\right)\right] \ell^{\prime}\left(\ell^{\prime}+1\right)\left\{\begin{array}{c}
V_{\ell^{\prime}}^{m} \\
0
\end{array}\right\} \\
& \left.+\left[-20 \frac{h_{4}}{q}+\frac{36 h_{2}}{35 q}\left(\frac{h_{2}}{q}+5 \frac{d h_{2}}{d q}\right)\right]\left\{\begin{array}{c}
\frac{d V_{\ell^{\prime}}^{m}}{d q} \\
0
\end{array}\right\}+\frac{144 h_{2}^{2}}{35 q}\left\{\begin{array}{c}
\frac{d^{2} V_{\ell^{\prime}}^{m}}{d q^{2}} \\
0
\end{array}\right\}\right\} \\
& +\sum_{\ell^{\prime}=|\ell-2|}^{\ell+2}\left[\begin{array}{ccc}
\ell & 2 & \ell^{\prime} \\
0 & 1 & -1 \\
m & 0 & m
\end{array}\right] 2 \sqrt{3} L_{0}^{\ell^{\prime}}\left[\frac{h_{2}}{q^{2}}\left\{\begin{array}{c}
U_{\ell^{\prime}}^{m} \\
0
\end{array}\right\}+\frac{1}{q}\left(\frac{d h_{2}}{d q}-\frac{h_{2}}{q}-3 \frac{h_{2}^{2}}{q^{2}}\right)\left\{\begin{array}{c}
V_{\ell^{\prime}}^{m} \\
0
\end{array}\right\}\right. \\
& \left.-\frac{h_{2}}{q}\left\{\begin{array}{c}
3 \frac{d V_{\ell^{\prime}}^{m}}{d q} \\
-i \frac{d W_{\ell^{\prime}}^{m}}{d q}
\end{array}\right\}+\frac{1}{q}\left(\frac{h_{2}}{q}-\frac{d h_{2}}{d q}\right)\left\{\begin{array}{c}
0 \\
i W_{\ell^{\prime}}^{m}
\end{array}\right\}\right]
\end{aligned}
$$




$$
\begin{aligned}
& +\sum_{\ell^{\prime}=|\ell-4|}^{\ell+4}\left[\begin{array}{ccc}
\ell & 4 & \ell^{\prime} \\
0 & 1 & -1 \\
m & 0 & m
\end{array}\right] 2 \sqrt{10} L_{0}^{\ell^{\prime}}\left[\frac{h_{4}}{q^{2}}\left\{\begin{array}{c}
U_{\ell^{\prime \prime}}^{m} \\
0
\end{array}\right\}+\frac{1}{q}\left(\frac{d h_{4}}{d q}-\frac{h_{4}}{q}\right)\left\{\begin{array}{c}
V_{\ell^{\prime}}^{m} \\
-i W_{\ell^{\prime}}^{m}
\end{array}\right\}-\frac{h_{4}}{q}\left\{\begin{array}{c}
3 \frac{d V_{\ell^{\prime}}^{m}}{d q} \\
-i \frac{d W_{\ell^{\prime}}^{m}}{d q}
\end{array}\right\}\right] \\
& +\sum_{\ell^{\prime}=|\ell-2|}^{\ell+2} \sum_{\ell^{\prime \prime}=\left|\ell^{\prime}-2\right|}^{\ell^{\prime}+2}\left[\begin{array}{ccc}
\ell & 2 & \ell^{\prime} \\
0 & 0 & 0 \\
m & 0 & m
\end{array}\right]\left[\begin{array}{ccc}
\ell^{\prime} & 2 & \ell^{\prime \prime} \\
0 & 1 & -1 \\
m & 0 & m
\end{array}\right] 2 \sqrt{3} L_{0}^{\ell^{\prime \prime}} \\
& \times\left\{\frac{h_{2}}{q^{2}}\left(-\frac{d h_{2}}{d q}-\frac{2 h_{2}}{q}\right)\left\{\begin{array}{c}
U_{\ell^{\prime \prime}}^{m} \\
0
\end{array}\right\}+\left[\frac{h_{2}}{q}\left(-\frac{d^{2} h_{2}}{d q^{2}}+\frac{2}{q} \frac{d h_{2}}{d q}+\frac{5 h_{2}}{q^{2}}\right)-\frac{3}{q}\left(\frac{d h_{2}}{d q}\right)^{2}\right]\left\{\begin{array}{c}
V_{\ell^{\prime \prime}}^{m} \\
0
\end{array}\right\}+\frac{h_{2}}{q}\left(5 \frac{d h_{2}}{d q}+3 \frac{h_{2}}{q}\right)\left\{\begin{array}{c}
\frac{d V_{\ell^{\prime \prime}}^{m}}{d q} \\
0
\end{array}\right\}\right. \\
& \left.-\frac{h_{2}}{q}\left(3 \frac{d h_{2}}{d q}+\frac{h_{2}}{q}\right)\left\{\begin{array}{c}
0 \\
i \frac{d W_{\ell^{\prime \prime}}^{m}}{d q}
\end{array}\right\}-\left[\frac{h_{2}}{q}\left(-\frac{d^{2} h_{2}}{d q^{2}}+\frac{1}{q} \frac{d h_{2}}{d q}+\frac{2 h_{2}}{q^{2}}\right)-\frac{3}{q}\left(\frac{d h_{2}}{d q}\right)^{2}\right]\left\{\begin{array}{c}
0 \\
i W_{\ell^{\prime \prime}}^{m}
\end{array}\right\}\right\} \\
& \left.+\sum_{\ell^{\prime}=|\ell-2|}^{\ell+2} \sum_{\ell^{\prime \prime}=\left|\ell^{\prime}-2\right|}^{\ell^{\prime}+2}\left[\begin{array}{ccc}
\ell & 2 & \ell^{\prime} \\
0 & 1 & -1 \\
m & 0 & m
\end{array}\right]\left[\begin{array}{ccc}
\ell^{\prime} & 2 & \ell^{\prime \prime} \\
0 & 1 & -1 \\
m & 0 & m
\end{array}\right] 12 L_{0}^{\ell} L_{0}^{\ell^{\prime \prime}} \frac{h_{2}^{2}}{q^{3}}\left\{\begin{array}{c}
0 \\
-i W_{\ell^{\prime \prime}}^{m}
\end{array}\right\}\right\} \\
& +\frac{d^{2} \tilde{\phi}_{0}}{d q^{2}}\left\{\left\{1-3 \frac{d h_{0}}{d q}+\frac{6}{5}\left[\frac{h_{2}^{2}}{q^{2}}+\left(\frac{d h_{2}}{d q}\right)^{2}\right]\right\} U_{\ell}^{m}+\frac{6 h_{2}^{2}}{5 q} \frac{d V_{\ell}^{m}}{d q}\right. \\
& +\sum_{\ell^{\prime}=|\ell-2|}^{\ell+2}\left[\begin{array}{ccc}
\ell & 2 & \ell^{\prime} \\
0 & 0 & 0 \\
m & 0 & m
\end{array}\right]\left\{\left[-3 \frac{d h_{2}}{d q}+\frac{2}{7}\left(\frac{h_{2}^{2}}{q^{2}}+6\left(\frac{d h_{2}}{d q}\right)^{2}\right)\right]\left\{\begin{array}{c}
U_{\ell^{\prime}}^{m} \\
0
\end{array}\right\}+\frac{2 h_{2}^{2}}{7 q}\left\{\begin{array}{c}
\frac{d V_{\ell^{\prime}}^{m}}{d q} \\
0
\end{array}\right\}\right\} \\
& +\sum_{\ell^{\prime}=|\ell-4|}^{\ell+4}\left[\begin{array}{ccc}
\ell & 4 & \ell^{\prime} \\
0 & 0 & 0 \\
m & 0 & m
\end{array}\right]\left\{\left[-3 \frac{d h_{4}}{d q}+\frac{36}{35}\left(-2 \frac{h_{2}^{2}}{q^{2}}+3\left(\frac{d h_{2}}{d q}\right)^{2}\right)\right]\left\{\begin{array}{c}
U_{\ell^{\prime}}^{m} \\
0
\end{array}\right\}-\frac{72 h_{2}^{2}}{35 q}\left\{\begin{array}{c}
\frac{d V_{\ell^{\prime}}^{m}}{d q} \\
0
\end{array}\right\}\right\} \\
& +\sum_{\ell^{\prime}=|\ell-2|}^{\ell+2}\left[\begin{array}{ccc}
\ell & 2 & \ell^{\prime} \\
0 & 1 & -1 \\
m & 0 & m
\end{array}\right] 2 \sqrt{3} L_{0}^{\ell^{\prime}} \frac{h_{2}}{q}\left\{\begin{array}{c}
V_{\ell^{\prime}}^{m} \\
-i W_{\ell^{\prime}}^{m}
\end{array}\right\} \\
& +\sum_{\ell^{\prime}=|\ell-4|}^{\ell+4}\left[\begin{array}{ccc}
\ell & 4 & \ell^{\prime} \\
0 & 1 & -1 \\
m & 0 & m
\end{array}\right] 2 \sqrt{10} L_{0}^{\ell^{\prime}} \frac{h_{4}}{q}\left\{\begin{array}{c}
V_{\ell^{\prime}}^{m} \\
-i W_{\ell^{\prime}}^{m}
\end{array}\right\} \\
& \left.+\sum_{\ell^{\prime}=|\ell-2|}^{\ell+2} \sum_{\ell^{\prime \prime}=\left|\ell^{\prime}-2\right|}^{\ell^{\prime}+2}\left[\begin{array}{ccc}
\ell & 2 & \ell^{\prime} \\
0 & 0 & 0 \\
m & 0 & m
\end{array}\right]\left[\begin{array}{ccc}
\ell^{\prime} & 2 & \ell^{\prime \prime} \\
0 & 1 & -1 \\
m & 0 & m
\end{array}\right] 2 \sqrt{3} L_{0}^{\ell^{\prime \prime}} \frac{h_{2}}{q}\left(3 \frac{d h_{2}}{d q}+\frac{h_{2}}{q}\right)\left\{\begin{array}{c}
-V_{\ell^{\prime \prime}}^{m} \\
i W_{\ell^{\prime \prime}}^{m}
\end{array}\right\}\right\} \text {. }
\end{aligned}
$$

In the double sums over both $\ell^{\prime}$ and $\ell^{\prime \prime}$, which contain terms of the second order in $h$, symbols like $\left\{\begin{array}{c}-V_{\ell^{\prime \prime}}^{m} \\ i W_{\ell^{\prime \prime}}^{m}\end{array}\right\}$ mean that the upper line must be chosen if both $\left|\ell-\ell^{\prime}\right|$ and $\left|\ell^{\prime}-\ell^{\prime \prime}\right|$ are even, odd, or zero, and that the lower line must be chosen if $\left|\ell-\ell^{\prime}\right|$ is even or zero and $\left|\ell^{\prime}-\ell^{\prime \prime}\right|$ is odd, or if $\left|\ell-\ell^{\prime}\right|$ is odd and $\left|\ell^{\prime}-\ell^{\prime \prime}\right|$ is even or zero. Moreover, in $\eta_{2}^{U m} \ell$, the derivatives of the harmonic components appearing in the sums over $\ell^{\prime}$ must be computed to first order in $h$ when they are multiplied by a coefficient of first order in $h$.

In order to obtain the radial derivative of $Q^{m}$, we form the combination

$\frac{1}{\sin \chi}\left\{\frac{\partial}{\partial \chi}\left[\sin \chi\left(\delta t_{; q}^{\chi q}+\delta t_{; \chi}^{\chi \chi}+\delta t_{; \nu}^{\chi v}\right)\right]+\frac{\partial}{\partial v}\left[\sin \chi\left(\delta t_{; q}^{v q}+\delta t_{; \chi}^{\nu \chi}+\delta t_{; v}^{\nu v}\right)\right]\right\}$. 
It gives

$$
\begin{aligned}
& \ell(\ell+1) \frac{1}{q} \frac{d Q_{\ell}^{m}}{d q}=-\rho_{0}\left(\eta_{1 \ell}^{V^{m}}+\eta_{2 \ell}^{V_{\ell}^{m}}\right)+\left(-3+2 \frac{h_{2}}{q}\right) \ell(\ell+1) \frac{Q_{\ell}^{m}}{q^{2}}-\ell(\ell+1)\left\{\left[2\left(\lambda_{0}+\mu_{0}\right)+2 \frac{h_{2}}{q} \mu_{0}\right] \frac{U_{\ell}^{m}}{q^{3}}+\frac{\lambda_{0}}{q^{2}} \frac{d U_{\ell}^{m}}{d q}\right\} \\
& +\ell(\ell+1)\left[\ell(\ell+1) \frac{\beta_{0}}{q}-2 \frac{\mu_{0}}{q}+2 \frac{h_{2}}{q}\left(\frac{\mu_{0}}{q}-\frac{d \mu_{0}}{d q}\right)\right] \frac{V_{\ell}^{m}}{q^{2}}+\left[6 m^{2}-2 \ell(\ell+1)\right] \frac{h_{2}}{q^{3}} \mu_{0} \frac{d V_{\ell}^{m}}{d q} \\
& +\sum_{\ell^{\prime}=|\ell-2|}^{\ell+2}\left[\begin{array}{ccc}
\ell & 2 & \ell^{\prime} \\
0 & 0 & 0 \\
m & 0 & m
\end{array}\right]\left\{\left(-2 \frac{h_{2}}{q^{2}}+\frac{3}{q} \frac{d h_{2}}{d q}+\frac{d^{2} h_{2}}{d q^{2}}\right) \frac{\ell^{\prime}\left(\ell^{\prime}+1\right)}{q}\left\{\begin{array}{c}
Q_{\ell^{\prime}}^{m} \\
0
\end{array}\right\}\right. \\
& +\left[18 \frac{h_{2}}{q}+6 \frac{d h_{2}}{d q}+\ell^{\prime}\left(\ell^{\prime}+1\right)\left(\frac{h_{2}}{q}+2 \frac{d h_{2}}{d q}\right)\right] \frac{1}{q}\left\{\begin{array}{c}
\frac{d Q_{\ell^{\prime}}^{m}}{d q} \\
0
\end{array}\right\} \\
& +6 \frac{h_{2}}{q}\left\{\begin{array}{c}
\frac{d^{2} Q_{\ell^{\prime}}^{m}}{d q^{2}} \\
0
\end{array}\right\}+\ell^{\prime}\left(\ell^{\prime}+1\right) \frac{h_{2}}{q^{4}}\left(6 \lambda_{0}+14 \mu_{0}\right)\left\{\begin{array}{c}
U_{\ell^{\prime}}^{m} \\
0
\end{array}\right\} \\
& +\frac{\lambda_{0}}{q^{2}}\left[\ell^{\prime}\left(\ell^{\prime}+1\right)\left(2 \frac{h_{2}}{q}+\frac{d h_{2}}{d q}\right)+6 \frac{d h_{2}}{d q}\right]\left\{\begin{array}{c}
\frac{d U_{\ell^{\prime}}^{m}}{d q} \\
0
\end{array}\right\}+6 \lambda_{0} \frac{h_{2}}{q^{2}}\left\{\begin{array}{c}
\frac{d^{2} U_{\ell^{\prime}}^{m}}{d q^{2}} \\
0
\end{array}\right\} \\
& -\ell^{\prime}\left(\ell^{\prime}+1\right)\left[3 \ell^{\prime}\left(\ell^{\prime}+1\right) \frac{\beta_{0}}{q}+2 \frac{\mu_{0}}{q}+\left(6 \frac{d \lambda_{0}}{d q}+4 \frac{d \mu_{0}}{d q}\right)\right] \frac{h_{2}}{q^{3}}\left\{\begin{array}{c}
V_{\ell^{\prime}}^{m} \\
0
\end{array}\right\} \\
& \left.-\left[\ell^{\prime}\left(\ell^{\prime}+1\right)\left(12 \lambda_{0}+4 \mu_{0}\right)+6\left(6 \lambda_{0}+10 \mu_{0}\right)\right] \frac{h_{2}}{q^{3}}\left\{\begin{array}{c}
\frac{d V_{\ell^{\prime}}^{m}}{d q} \\
0
\end{array}\right\}\right\} \\
& +\sum_{\ell^{\prime}=|\ell-2|}^{\ell+2}\left[\begin{array}{ccc}
\ell & 2 & \ell^{\prime} \\
0 & 1 & -1 \\
m & 0 & m
\end{array}\right] 2 \sqrt{3} L_{0}^{\ell^{\prime}} \\
& \times\left\{\left[(4-2 \ell(\ell+1)) \frac{h_{2}}{q^{2}}+\frac{3}{q} \frac{d h_{2}}{d q}+\frac{d^{2} h_{2}}{d q^{2}}\right]\left\{\begin{array}{c}
\frac{Q_{\ell^{\prime}}^{m}}{q} \\
0
\end{array}\right\}+\left(4 \frac{h_{2}}{q^{2}}+\frac{3}{q} \frac{d h_{2}}{d q}\right)\left\{\begin{array}{c}
\frac{d Q_{\ell^{\prime}}^{m}}{d q} \\
0
\end{array}\right\}+\frac{h_{2}}{q}\left\{\begin{array}{c}
\frac{d^{2} Q_{\ell^{\prime}}^{m}}{d q^{2}} \\
i \frac{d^{2} R_{\ell^{\prime}}^{m}}{d q^{2}}
\end{array}\right\}\right. \\
& +\left(2 \frac{h_{2}}{q^{2}}-\frac{3}{q} \frac{d h_{2}}{d q}-\frac{d^{2} h_{2}}{d q^{2}}\right)\left\{\begin{array}{c}
0 \\
i \frac{R_{\ell^{\prime}}^{m}}{q}
\end{array}\right\}+\left(2 \frac{h_{2}}{q^{2}}-\frac{1}{q} \frac{d h_{2}}{d q}\right)\left\{\begin{array}{c}
0 \\
i \frac{d R_{\ell^{\prime}}^{m}}{d q}
\end{array}\right\} \\
& +\left\{6 \lambda_{0}+2[\ell(\ell+1)+4] \mu_{0}\right\} \frac{h_{2}}{q^{4}}\left\{\begin{array}{c}
U_{\ell^{\prime}}^{m} \\
0
\end{array}\right\}+\lambda_{0} \frac{h_{2}}{q^{2}}\left\{\begin{array}{c}
\frac{d^{2} U_{\ell^{\prime}}^{m}}{d q^{2}}+\frac{2}{q} \frac{d U_{\ell^{\prime}}^{m}}{d q} \\
0
\end{array}\right\} \\
& +\left[2 \frac{d \mu_{0}}{d q}-4 \frac{\mu_{0}}{q}-\ell^{\prime}\left(\ell^{\prime}+1\right)\left(3 \frac{\lambda_{0}}{q}+8 \frac{\mu_{0}}{q}+\frac{d \beta_{0}}{d q}\right)\right] \frac{h_{2}}{q^{3}}\left\{\begin{array}{c}
V_{\ell^{\prime}}^{m} \\
0
\end{array}\right\} \\
& +\left\{\ell^{\prime}\left(\ell^{\prime}+1\right) \frac{d \mu_{0}}{d q}+\left[2+3 \ell^{\prime}\left(\ell^{\prime}+1\right)\right] \frac{\mu_{0}}{q}\right\} \frac{h_{2}}{q^{3}}\left\{\begin{array}{c}
0 \\
i W_{\ell^{\prime}}^{m}
\end{array}\right\} \\
& \left.-\left\{\left[12+\ell^{\prime}\left(\ell^{\prime}+1\right)\right] \lambda_{0}+14 \mu_{0}+2 \ell(\ell+1) \beta_{0}\right\} \frac{h_{2}}{q^{3}}\left\{\begin{array}{c}
\frac{d V_{\ell^{\prime}}^{m}}{d q} \\
0
\end{array}\right\}+\left[6+\ell^{\prime}\left(\ell^{\prime}+1\right)\right] \mu_{0} \frac{h_{2}}{q^{3}}\left\{\begin{array}{c}
0 \\
i \frac{d W_{\ell^{\prime}}^{m}}{d q}
\end{array}\right\}\right\} \\
& +\sum_{\ell^{\prime}=|\ell-1|}^{\ell+1} \sum_{\ell^{\prime \prime}=\left|\ell^{\prime}-1\right|}^{\ell^{\prime}+1}\left[\begin{array}{ccc}
\ell^{\prime} & 1 & \ell^{\prime \prime} \\
0 & 1 & -1 \\
m & 0 & m
\end{array}\right]\left(2 L_{0}^{\ell^{\prime}}\left[\begin{array}{ccc}
\ell & 1 & \ell^{\prime} \\
0 & 1 & -1 \\
m & 0 & m
\end{array}\right]-\left[\begin{array}{ccc}
\ell & 1 & \ell^{\prime} \\
0 & 0 & 0 \\
m & 0 & m
\end{array}\right]\right) \\
& \times 12 \frac{h_{2}}{q^{3}} L_{0}^{\ell^{\prime \prime}}\left[\left\{\begin{array}{c}
Q_{\ell^{\prime \prime}}^{m} \\
0
\end{array}\right\}-\mu_{0}\left\{\begin{array}{c}
\frac{U_{\ell^{\prime \prime}}^{m}}{q}-\frac{V_{\ell^{\prime \prime}}^{m}}{q} \\
i \frac{d W_{\ell^{\prime \prime}}^{m}}{d q}
\end{array}\right\}-\frac{d \mu_{0}}{d q}\left\{\begin{array}{c}
V_{\ell^{\prime \prime}}^{m} \\
0
\end{array}\right\}\right]
\end{aligned}
$$




$$
\begin{aligned}
& +\sum_{\ell^{\prime}=|\ell-1|}^{\ell+1}\left[\begin{array}{ccc}
\ell & 1 & \ell^{\prime} \\
0 & 0 & 0 \\
m & 0 & m
\end{array}\right] 6 m \frac{h_{2}}{q^{3}} \frac{d \mu_{0}}{d q}\left\{\begin{array}{c}
0 \\
i W_{\ell^{\prime}}^{m}
\end{array}\right\} \\
& -\sum_{\ell^{\prime}=|\ell-1|}^{\ell+1}\left[\begin{array}{ccc}
\ell & 1 & \ell^{\prime} \\
0 & 1 & -1 \\
m & 0 & m
\end{array}\right] 12 m L_{0}^{\ell^{\prime}} \frac{h_{2}}{q^{3}}\left\{i\left[R_{\ell^{\prime}}^{m}+\left(\frac{d \mu_{0}}{d q}+\frac{\mu_{0}}{q}\right) W_{\ell^{\prime}}^{m}\right]\right\},
\end{aligned}
$$

where

$$
\begin{aligned}
& \eta_{1 \ell}^{V^{m}}=\frac{\omega^{2}}{q}\left\{\ell(\ell+1) V_{\ell}^{m}+\sum_{\ell^{\prime}=\ell-2 \mid}^{\ell+2}\left[\begin{array}{ccc}
\ell & 2 & \ell^{\prime} \\
0 & 0 & 0 \\
m & 0 & m
\end{array}\right] h_{2}\left\{\begin{array}{c}
-6 \frac{d V_{\ell^{\prime}}^{m}}{d q}-\ell^{\prime}\left(\ell^{\prime}+1\right) \frac{V_{\ell^{\prime}}^{m}}{q} \\
0
\end{array}\right\}\right. \\
& \left.+\sum_{\ell^{\prime}=|\ell-2|}^{\ell+2}\left[\begin{array}{ccc}
\ell & 2 & \ell^{\prime} \\
0 & 1 & -1 \\
m & 0 & m
\end{array}\right] 2 \sqrt{3} h_{2} L_{0}^{\ell^{\prime}}\left\{\begin{array}{c}
\frac{V_{\ell^{\prime}}^{m}}{q}-\frac{d V_{\ell^{\prime}}^{m}}{d q} \\
i\left(\frac{W_{\ell^{\prime}}^{m}}{q}+\frac{d W_{\ell^{\prime}}^{m}}{d q}\right)
\end{array}\right\}\right\} \\
& +\frac{2}{q} \omega \Omega_{0}\left\{\sum_{\ell^{\prime}=|\ell-1|}^{\ell+1}\left[\begin{array}{ccc}
\ell & 1 & \ell^{\prime} \\
-1 & 0 & -1 \\
m & 0 & m
\end{array}\right] 2 L_{0}^{\ell} L_{0}^{\ell^{\prime}}\left\{\begin{array}{c}
\left(U_{\ell^{\prime}}^{m}+V_{\ell^{\prime}}^{m}\right) \\
-i W_{\ell^{\prime}}^{m}
\end{array}\right\}\right. \\
& +\sum_{\ell^{\prime}=|\ell-2|}^{\ell+2} \sum_{\ell^{\prime \prime}=\left|\ell^{\prime}-1\right|}^{\ell^{\prime}+1}\left[\begin{array}{ccc}
\ell & 2 & \ell^{\prime} \\
0 & 0 & 0 \\
m & 0 & m
\end{array}\right]\left[\begin{array}{ccc}
\ell^{\prime} & 1 & \ell^{\prime \prime} \\
-1 & 0 & -1 \\
m & 0 & m
\end{array}\right] 2 \frac{h_{2}}{q} L_{0}^{\ell^{\prime}} L_{0}^{\ell^{\prime \prime}}\left\{\begin{array}{c}
\left(U_{\ell^{\prime \prime}}^{m}+V_{\ell^{\prime \prime}}^{m}\right) \\
i W_{\ell^{\prime \prime}}^{m}
\end{array}\right\} \\
& +\sum_{\ell^{\prime}=|\ell-1|}^{\ell+1}\left[\begin{array}{ccc}
\ell & 1 & \ell^{\prime} \\
0 & 1 & -1 \\
m & 0 & m
\end{array}\right] 2 h_{2} L_{0}^{\ell^{\prime}}\left\{\begin{array}{c}
\frac{d V_{\ell^{\prime}}^{m}}{d q}-\frac{V_{\ell^{\prime}}^{m}}{q} \\
i\left(\frac{W_{\ell^{\prime}}^{m}}{q}+\frac{d W_{\ell^{\prime}}^{m}}{d q}\right)
\end{array}\right\} \\
& +\sum_{\ell^{\prime}=|\ell-2| \ell^{\prime \prime}=\left|\ell^{\prime}-1\right|}^{\ell+2} \sum_{\ell^{\prime}+1}^{\ell^{\prime}}\left[\begin{array}{ccc}
\ell & 2 & \ell^{\prime} \\
0 & 0 & 0 \\
m & 0 & m
\end{array}\right]\left[\begin{array}{ccc}
\ell^{\prime} & 1 & \ell^{\prime \prime} \\
0 & 1 & -1 \\
m & 0 & m
\end{array}\right] 4 h_{2} L_{0}^{\ell^{\prime \prime}}\left\{\begin{array}{c}
\frac{d V_{\ell^{\prime \prime}}^{m}}{d q}-\frac{V_{\ell^{\prime \prime}}^{m}}{q} \\
i\left(\frac{W_{\ell^{\prime \prime}}^{m}}{q}+\frac{d W_{\ell^{\prime \prime}}^{m}}{d q}\right)
\end{array}\right\} \\
& \left.+\sum_{\ell^{\prime}=|\ell-1|}^{\ell+1}\left[\begin{array}{ccc}
\ell & 1 & \ell^{\prime} \\
0 & 0 & 0 \\
m & 0 & m
\end{array}\right] \frac{6}{5} h_{2}\left\{\begin{array}{c}
0 \\
i \frac{d W_{e^{\prime}}^{m}}{d q}
\end{array}\right\}+\sum_{\ell^{\prime}=|\ell-3|}^{\ell+3}\left[\begin{array}{ccc}
\ell & 3 & \ell^{\prime} \\
0 & 0 & 0 \\
m & 0 & m
\end{array}\right] \frac{24}{5} h_{2}\left\{\begin{array}{c}
0 \\
i \frac{d W_{\ell^{\prime}}^{m}}{d q}
\end{array}\right\}\right\}
\end{aligned}
$$

and

$$
\begin{aligned}
\eta_{2 \ell}^{V^{m}}=[\ell(\ell & \left.+1)\left(-1+\frac{3}{5} \frac{h_{2}^{2}}{q^{2}}\right)-3 m^{2} \frac{h_{2}^{2}}{q^{2}}\right] \frac{\phi_{\ell}^{m}}{q^{2}} \\
& +\sum_{\ell^{\prime}=|\ell-2|}^{\ell+2}\left[\begin{array}{ccc}
\ell & 2 & \ell^{\prime} \\
0 & 0 & 0 \\
m & 0 & m
\end{array}\right]\left\{\left[\ell^{\prime}\left(\ell^{\prime}+1\right) \frac{h_{2}}{q^{3}}\left(2-\frac{4}{7} \frac{h_{2}}{q}\right)-6 m^{2} \frac{h_{2}^{2}}{q^{4}}\right]\left\{\begin{array}{c}
\phi_{\ell^{\prime}}^{m} \\
0
\end{array}\right\}\right. \\
& \left.+\frac{h_{2}}{q^{2}}\left(6-\frac{20}{7} \frac{h_{2}}{q}\right)\left\{\begin{array}{c}
g_{\ell^{\prime}}^{m}-4 \pi \mathrm{G} \rho_{0} U_{\ell^{\prime}}^{m} \\
0
\end{array}\right\}+4 \pi G \rho_{0} \frac{h_{2}^{2}}{q^{4}}\left[\frac{2}{7} \ell^{\prime}\left(\ell^{\prime}+1\right)-6 m^{2}\right]\left\{\begin{array}{c}
V_{\ell^{\prime}}^{m} \\
0
\end{array}\right\}\right\} \\
& +\sum_{\ell^{\prime}=|\ell-4|}^{\ell+4}\left[\begin{array}{ccc}
\ell & 4 & \ell^{\prime} \\
0 & 0 & 0 \\
m & 0 & m
\end{array}\right]\left[\frac{\ell^{\prime}\left(\ell^{\prime}+1\right)}{q^{2}}\left(2 \frac{h_{4}}{q}-\frac{18}{5} \frac{h_{2}^{2}}{q^{2}}\right)\left\{\begin{array}{c}
\phi_{\ell^{\prime}}^{m} \\
0
\end{array}\right\}\right.
\end{aligned}
$$




$$
\begin{aligned}
& \left.+\left(20 \frac{h_{4}}{q^{2}}-\frac{72}{7} \frac{h_{2}^{2}}{q^{3}}\right)\left\{\begin{array}{c}
g_{\ell^{\prime}}^{m}-4 \pi \mathrm{G} \rho_{0} U_{\ell^{\prime}}^{m} \\
0
\end{array}\right\}-\ell^{\prime}\left(\ell^{\prime}+1\right) 4 \pi G \rho_{0} \frac{72}{35} \frac{h_{2}^{2}}{q^{3}}\left\{\begin{array}{c}
V_{\ell^{\prime}}^{m} \\
0
\end{array}\right\}\right] \\
& +\sum_{\ell^{\prime}=|\ell-2|}^{\ell+2}\left[\begin{array}{ccc}
\ell & 2 & \ell^{\prime} \\
0 & 1 & -1 \\
m & 0 & m
\end{array}\right] 2 \sqrt{3} L_{0}^{\ell^{\prime}} \frac{h_{2}}{q^{2}}\left[\left\{\begin{array}{c}
2 \frac{\phi_{\ell^{\prime}}^{m}}{q}+g_{\ell^{\prime}}^{m}-4 \pi \mathrm{G} \rho_{0} U_{\ell^{\prime}}^{m} \\
0
\end{array}\right\}+\frac{h_{2}}{q}\left\{\begin{array}{c}
2 \frac{\phi_{\ell^{\prime}}^{m}}{q}+4 \pi \mathrm{G} \rho_{0} V_{\ell^{\prime}}^{m} \\
-4 \pi \mathrm{G} \rho_{0} i W_{\ell^{\prime}}^{m}
\end{array}\right\}\right] \\
& +\sum_{\ell^{\prime}=|\ell-4|}^{\ell+4}\left[\begin{array}{ccc}
\ell & 4 & \ell^{\prime} \\
0 & 1 & -1 \\
m & 0 & m
\end{array}\right] 2 \sqrt{10} L_{0}^{\ell^{\prime}} \frac{h_{4}}{q^{2}}\left\{\begin{array}{c}
2 \frac{\phi_{\ell^{\prime}}^{m}}{q}+g_{\ell^{\prime}}^{m}-4 \pi \mathrm{G} \rho_{0} U_{\ell^{\prime}}^{m} \\
0
\end{array}\right\} \\
& -\sum_{\ell^{\prime}=|\ell-2|}^{\ell+2} \sum_{\ell^{\prime \prime}=\left|\ell^{\prime}-2\right|}^{\ell^{\prime}+2}\left[\begin{array}{ccc}
\ell & 2 & \ell^{\prime} \\
0 & 0 & 0 \\
m & 0 & m
\end{array}\right]\left[\begin{array}{ccc}
\ell^{\prime} & 2 & \ell^{\prime \prime} \\
0 & 1 & -1 \\
m & 0 & m
\end{array}\right] 2 \sqrt{3} L_{0}^{\ell^{\prime \prime}} \frac{h_{2}^{2}}{q^{3}}\left\{\begin{array}{c}
14 \frac{\phi_{\ell^{\prime \prime}}^{m}}{q}-5 \pi \mathrm{G} \rho_{0} V_{\ell^{\prime \prime}}^{m} \\
2 \pi \mathrm{G} \rho_{0} i W_{\ell^{\prime \prime}}^{m}
\end{array}\right\} \\
& -\sum_{\ell^{\prime}=|\ell-2|}^{\ell+2} \sum_{\ell^{\prime \prime}=\left|\ell^{\prime}-2\right|}^{\ell^{\prime}+2}\left[\begin{array}{ccc}
\ell & 2 & \ell^{\prime} \\
0 & 1 & -1 \\
m & 0 & m
\end{array}\right]\left[\begin{array}{ccc}
\ell^{\prime} & 2 & \ell^{\prime \prime} \\
0 & 1 & -1 \\
m & 0 & m
\end{array}\right] 12 L_{0}^{\ell^{\prime}} L_{0}^{\ell^{\prime \prime}} \frac{h_{2}^{2}}{q^{3}}\left\{\begin{array}{c}
0 \\
4 \pi \mathrm{G} \rho_{0} i W_{\ell^{\prime \prime}}^{m}
\end{array}\right\} \\
& +\frac{d \tilde{\phi}_{0}}{d q}\left\{\left[-\ell(\ell+1)\left[1+\frac{1}{5}\left(3 \frac{h_{2}^{2}}{q^{2}}+\left(\frac{d h_{2}}{d q}\right)^{2}\right)\right]+\frac{6}{5}\left(-h_{2} \frac{d^{2} h_{2}}{d q^{2}}+\frac{h_{2}}{q} \frac{d h_{2}}{d q}\right)\right] \frac{U_{\ell}^{m}}{q^{2}}+9 \frac{h_{2}}{q}\left(\frac{h_{2}}{q}+\frac{d h_{2}}{d q}\right)\left[m^{2}-\frac{2}{5} \ell(\ell+1)\right] \frac{V_{\ell}^{m}}{q^{2}}\right. \\
& +\frac{h_{2}^{2}}{q^{2}}\left[\frac{12}{5} \ell(\ell+1)-3 m^{2}\right] \frac{1}{q} \frac{d V_{\ell}^{m}}{d q} \\
& +\sum_{\ell^{\prime}=|\ell-2|}^{\ell+2}\left[\begin{array}{ccc}
\ell & 2 & \ell^{\prime} \\
0 & 0 & 0 \\
m & 0 & m
\end{array}\right]\left\{\left[6 \frac{d h_{2}}{d q}-12 \frac{h_{2}}{q}+\ell^{\prime}\left(\ell^{\prime}+1\right)\left(\frac{2 h_{2}}{q}+\frac{d h_{2}}{d q}\right)\right.\right. \\
& \left.-\frac{2}{7}\left(6 h_{2} \frac{d^{2} h_{2}}{d q^{2}}+9 \frac{h_{2}}{q} \frac{d h_{2}}{d q}+\left[10+\ell^{\prime}\left(\ell^{\prime}+1\right)\right]\left(\frac{d h_{2}}{d q}\right)^{2}-\left[10-3 \ell^{\prime}\left(\ell^{\prime}+1\right)\right] \frac{h_{2}^{2}}{q^{2}}\right)\right]\left\{\begin{array}{c}
\frac{U_{\ell^{\prime}}^{m}}{q^{2}} \\
0
\end{array}\right\} \\
& -\frac{10}{7} \frac{h_{2}}{q^{2}}\left(2 \frac{h_{2}}{q}+\frac{d h_{2}}{d q}\right)\left\{\begin{array}{c}
\frac{d U_{\ell^{\prime}}^{m}}{d q} \\
0
\end{array}\right\} \\
& +\left\{6 \ell^{\prime}\left(\ell^{\prime}+1\right) \frac{h_{2}}{q}+\left[\frac{6}{7} \ell^{\prime}\left(\ell^{\prime}+1\right)-18 m^{2}\right] \frac{h_{2}}{q} \frac{d h_{2}}{d q}-\left[\frac{4}{7} \ell^{\prime}\left(\ell^{\prime}+1\right)+18 m^{2}\right] \frac{h_{2}^{2}}{q^{2}}\right\}\left\{\begin{array}{c}
\frac{V_{\ell^{\prime}}^{m}}{q^{2}} \\
0
\end{array}\right\} \\
& \left.+\frac{h_{2}^{2}}{q^{3}}\left[\frac{4}{7} \ell^{\prime}\left(\ell^{\prime}+1\right)-18 m^{2}-8\right]\left\{\begin{array}{c}
\frac{d V_{\ell^{\prime}}^{m}}{d q} \\
0
\end{array}\right\}\right\} \\
& +\sum_{\ell^{\prime}=|\ell-4|}^{\ell+4}\left[\begin{array}{ccc}
\ell & 4 & \ell^{\prime} \\
0 & 0 & 0 \\
m & 0 & m
\end{array}\right]\left\{\left[20 \frac{d h_{4}}{d q}-40 \frac{h_{4}}{q}-\frac{18}{35}\left(6 h_{2} \frac{d^{2} h_{2}}{d q^{2}}+24 \frac{h_{2}}{q} \frac{d h_{2}}{d q}\right.\right.\right. \\
& \left.\left.+\left[20+\ell^{\prime}\left(\ell^{\prime}+1\right)\right]\left(\frac{d h_{2}}{d q}\right)^{2}-\left[20+3 \ell^{\prime}\left(\ell^{\prime}+1\right)\right] \frac{h_{2}^{2}}{q^{2}}\right)\right]\left\{\begin{array}{c}
\frac{U_{\ell^{\prime}}^{m}}{q^{2}} \\
0
\end{array}\right\}-\frac{36}{35} \frac{h_{2}}{q^{2}}\left(11 \frac{h_{2}}{q}+10 \frac{d h_{2}}{d q}\right)\left\{\begin{array}{c}
\frac{d U_{\ell^{\prime}}^{m}}{d q} \\
0
\end{array}\right\} \\
& \left.+\left[20 \frac{h_{4}}{q}+\frac{36}{35} \frac{h_{2}}{q}\left(\frac{h_{2}}{q}+6 \frac{d h_{2}}{d q}\right)\right] \ell^{\prime}\left(\ell^{\prime}+1\right)\left\{\begin{array}{c}
\frac{V_{\ell^{\prime}}^{m}}{q^{2}} \\
0
\end{array}\right\}+\frac{72}{35}\left[7-2 \ell^{\prime}\left(\ell^{\prime}+1\right)\right] \frac{h_{2}^{2}}{q^{3}}\left\{\begin{array}{c}
\frac{d V_{\ell^{\prime}}^{m}}{d q} \\
0
\end{array}\right\}\right\} \\
& +\sum_{\ell^{\prime}=|\ell-2|}^{\ell+2}\left[\begin{array}{ccc}
\ell & 2 & \ell^{\prime} \\
0 & 1 & -1 \\
m & 0 & m
\end{array}\right]\left\{2 \sqrt { 3 } L _ { 0 } ^ { \ell ^ { \prime } } \left\{\left(2 \frac{d h_{2}}{d q}-4 \frac{h_{2}}{q}\right)\left\{\begin{array}{c}
\frac{U_{\ell^{\prime}}^{m}}{q^{2}} \\
0
\end{array}\right\}+2 \frac{h_{2}^{2}}{q^{3}}\left\{\begin{array}{c}
\frac{d V_{\ell^{\prime}}^{m}}{d q} \\
i \frac{d W_{\ell^{\prime}}^{m}}{d q}
\end{array}\right\}\right.\right.
\end{aligned}
$$




$$
\begin{aligned}
& \left.\left.+\frac{h_{2}}{q}\left[\ell^{\prime}\left(\ell^{\prime}+1\right)-\ell(\ell+1)-3\left(\frac{d h_{2}}{d q}+\frac{h_{2}}{q}\right)\right]\left\{\begin{array}{c}
\frac{V_{\ell^{\prime}}^{m}}{q^{2}} \\
0
\end{array}\right\}+\frac{h_{2}}{q}\left[-\ell(\ell+1)+2\left(\frac{d h_{2}}{d q}-3 \frac{h_{2}}{q}\right)\right]\left\{\begin{array}{c}
0 \\
i \frac{W_{\ell^{\prime}}^{m}}{q^{2}}
\end{array}\right\}\right\}+\frac{h_{2}^{2}}{q^{3}} 6 m^{2}\left\{\begin{array}{c}
\frac{d V_{\ell^{\prime}}^{m}}{d q} \\
0
\end{array}\right\}\right\} \\
& +\sum_{\ell^{\prime}=|\ell-4|}^{\ell+4}\left[\begin{array}{ccc}
\ell & 4 & \ell^{\prime} \\
0 & 1 & -1 \\
m & 0 & m
\end{array}\right] 2 \sqrt{10} L_{0}^{\ell^{\prime}}\left[\left(2 \frac{d h_{4}}{d q}-4 \frac{h_{4}}{q}\right)\left\{\begin{array}{c}
\frac{U_{\ell^{\prime}}^{m}}{q^{2}} \\
0
\end{array}\right\}+\left[\ell^{\prime}\left(\ell^{\prime}+1\right)-\ell(\ell+1)\right] \frac{h_{4}}{q}\left\{\begin{array}{c}
\frac{V_{\ell^{\prime}}^{m}}{q^{2}} \\
0
\end{array}\right\}+\ell(\ell+1) \frac{h_{4}}{q}\left\{\begin{array}{c}
0 \\
i \frac{W_{\ell^{\prime}}^{m}}{q^{2}}
\end{array}\right\}\right] \\
& +\sum_{\ell^{\prime}=|\ell-2| \ell^{\prime \prime}=\left|\ell^{\prime}-2\right|}^{\ell+2} \sum^{\ell^{\prime}+2}\left[\begin{array}{ccc}
\ell & 2 & \ell^{\prime} \\
0 & 0 & 0 \\
m & 0 & m
\end{array}\right]\left[\begin{array}{ccc}
\ell^{\prime} & 2 & \ell^{\prime \prime} \\
0 & 1 & -1 \\
m & 0 & m
\end{array}\right] 2 \sqrt{3} L_{0}^{\ell^{\prime \prime}} \\
& \times\left\{\left[-h_{2} \frac{d^{2} h_{2}}{d q^{2}}+2 \frac{h_{2}}{q} \frac{d h_{2}}{d q}-4 \frac{h_{2}^{2}}{q^{2}}+4\left(\frac{d h_{2}}{d q}\right)^{2}\right]\left\{\begin{array}{c}
\frac{U_{\ell^{\prime \prime}}^{m}}{q^{2}} \\
0
\end{array}\right\}-\frac{h_{2}}{q^{2}}\left(2 \frac{h_{2}}{q}+\frac{d h_{2}}{d q}\right)\left\{\begin{array}{c}
\frac{d U_{\ell^{\prime \prime}}^{m}}{d q} \\
0
\end{array}\right\}-8 \frac{h_{2}^{2}}{q^{3}}\left\{\begin{array}{c}
2 \frac{d V_{l^{\prime \prime}}^{m}}{d q} \\
i \frac{d W_{\ell^{\prime \prime}}^{m}}{d q}
\end{array}\right\}\right. \\
& +\frac{h_{2}}{q^{3}}\left[\ell^{\prime}\left(\ell^{\prime}+1\right)\left(3 \frac{h_{2}}{q}+\frac{d h_{2}}{d q}\right)+3\left(3 \frac{d h_{2}}{d q}-\frac{h_{2}}{q}\right)\right]\left\{\begin{array}{c}
V_{\ell^{\prime \prime}}^{m} \\
0
\end{array}\right\} \\
& \left.+\left[-\ell^{\prime}\left(\ell^{\prime}+1\right)\left(3 \frac{h_{2}}{q}+\frac{d h_{2}}{d q}\right)+2\left(3 \frac{h_{2}}{q}-7 \frac{d h_{2}}{d q}\right)\right] \frac{h_{2}}{q^{3}}\left\{\begin{array}{c}
0 \\
i W_{\ell^{\prime \prime}}^{m}
\end{array}\right\}\right\} \\
& \left.-\sum_{\ell^{\prime}=|\ell-2| \ell^{\prime \prime}=\left|\ell^{\prime}-2\right|}^{\ell+2} \sum_{\ell^{\prime}+2}^{\ell^{\prime}}\left[\begin{array}{ccc}
\ell & 2 & \ell^{\prime} \\
0 & 1 & -1 \\
m & 0 & m
\end{array}\right]\left[\begin{array}{ccc}
\ell^{\prime} & 2 & \ell^{\prime \prime} \\
0 & 1 & -1 \\
m & 0 & m
\end{array}\right] 36 L_{0}^{\ell} L_{0}^{\ell^{\prime \prime}} \frac{h_{2}}{q^{3}}\left(\frac{h_{2}}{q}+\frac{d h_{2}}{d q}\right)\left\{\begin{array}{c}
0 \\
i W_{\ell^{\prime \prime}}^{m}
\end{array}\right\}\right\} \\
& +\frac{d^{2} \tilde{\phi}_{0}}{d q^{2}}\left\{\frac{8}{5} \frac{h_{2}}{q}\left(\frac{h_{2}}{q}+\frac{d h_{2}}{d q}\right) \frac{U_{\ell}^{m}}{q}-3\left[m^{2}-\frac{2}{5} \ell(\ell+1)\right] \frac{h_{2}^{2}}{q^{2}} \frac{V_{\ell}^{m}}{q}\right. \\
& +\sum_{\ell^{\prime}=|\ell-2|}^{\ell+2}\left[\begin{array}{ccc}
\ell & 2 & \ell^{\prime} \\
0 & 0 & 0 \\
m & 0 & m
\end{array}\right]\left\{6 \frac{h_{2}}{q^{2}}\left[-1+\frac{4}{7}\left(\frac{h_{2}}{q}+\frac{d h_{2}}{d q}\right)\right]\left\{\begin{array}{c}
U_{\ell^{\prime}}^{m} \\
0
\end{array}\right\}-2 \frac{h_{2}^{2}}{q^{3}}\left[3 m^{2}-\frac{1}{7} \ell^{\prime}\left(\ell^{\prime}+1\right)\right]\left\{\begin{array}{c}
V_{\ell^{\prime}}^{m} \\
0
\end{array}\right\}\right\} \\
& +\sum_{\ell^{\prime}=|\ell-4|}^{\ell+4}\left[\begin{array}{ccc}
\ell & 4 & \ell^{\prime} \\
0 & 0 & 0 \\
m & 0 & m
\end{array}\right]\left\{4\left[-5 \frac{h_{4}}{q}+\frac{18}{35} \frac{h_{2}}{q}\left(\frac{h_{2}}{q}+\frac{d h_{2}}{d q}\right)\right]\left\{\begin{array}{c}
\frac{U_{\ell^{\prime}}^{m}}{q} \\
0
\end{array}\right\}-\frac{72}{35} \ell^{\prime}\left(\ell^{\prime}+1\right) \frac{h_{2}^{2}}{q^{2}}\left\{\begin{array}{c}
\frac{V_{\ell^{\prime}}^{m}}{q} \\
0
\end{array}\right\}\right\} \\
& +\sum_{\ell^{\prime}=|\ell-2|}^{\ell+2}\left[\begin{array}{ccc}
\ell & 2 & \ell^{\prime} \\
0 & 1 & -1 \\
m & 0 & m
\end{array}\right] 2 \sqrt{3} L_{0}^{\ell^{\prime}}\left[-\frac{h_{2}}{q^{2}}\left\{\begin{array}{c}
U_{\ell^{\prime}}^{m} \\
0
\end{array}\right\}+2 \frac{h_{2}^{2}}{q^{3}}\left\{\begin{array}{c}
V_{\ell^{\prime}}^{m} \\
-i W_{\ell^{\prime}}^{m}
\end{array}\right\}\right] \\
& -\sum_{\ell^{\prime}=|\ell-4|}^{\ell+4}\left[\begin{array}{ccc}
\ell & 4 & \ell^{\prime} \\
0 & 1 & -1 \\
m & 0 & m
\end{array}\right] 2 \sqrt{10} L_{0}^{\ell^{\prime}} \frac{h_{4}}{q^{2}}\left\{\begin{array}{c}
U_{\ell^{\prime}}^{m} \\
0
\end{array}\right\} \\
& +\sum_{\ell^{\prime}=|\ell-2| \ell^{\prime \prime}=\left|\ell^{\prime}-2\right|}^{\ell+2} \sum_{\ell^{\prime}+2}\left[\begin{array}{ccc}
\ell & 2 & \ell^{\prime} \\
0 & 0 & 0 \\
m & 0 & m
\end{array}\right]\left[\begin{array}{ccc}
\ell^{\prime} & 2 & \ell^{\prime \prime} \\
0 & 1 & -1 \\
m & 0 & m
\end{array}\right] 2 \sqrt{3} L_{0}^{\ell^{\prime \prime}}\left[2 \frac{h_{2}}{q^{2}}\left(\frac{d h_{2}}{d q}+\frac{h_{2}}{q}\right)\left\{\begin{array}{c}
U_{\ell^{\prime \prime}}^{m} \\
0
\end{array}\right\}+8 \frac{h_{2}^{2}}{q^{3}}\left\{\begin{array}{c}
-V_{\ell^{\prime \prime}}^{m} \\
i W_{\ell^{\prime \prime}}^{m}
\end{array}\right\}\right] \\
& \left.+\sum_{\ell^{\prime}=|\ell-2| \ell^{\prime \prime}=\left|\ell^{\prime}-2\right|}^{\ell+2} \sum_{\ell^{\prime}+2}^{\ell^{\prime}}\left[\begin{array}{ccc}
\ell & 2 & \ell^{\prime} \\
0 & 1 & -1 \\
m & 0 & m
\end{array}\right]\left[\begin{array}{ccc}
\ell^{\prime} & 2 & \ell^{\prime \prime} \\
0 & 1 & -1 \\
m & 0 & m
\end{array}\right] 12 L_{0}^{\ell^{\prime \prime}} L_{0}^{\ell} \frac{h_{2}^{2}}{q^{3}}\left\{\begin{array}{c}
0 \\
i W_{\ell^{\prime \prime}}^{m}
\end{array}\right\}\right\}
\end{aligned}
$$

Finally, in order to obtain the radial derivative of $R_{\ell}^{m}$, we form the combination

$$
\frac{1}{\sin \chi}\left\{\frac{\partial}{\partial v}\left(\delta t_{; q}^{\chi q}+\delta t_{; \chi}^{\chi \chi}+\delta t_{; \nu}^{\chi v}\right)-\frac{\partial}{\partial \chi}\left[\sin ^{2} \chi\left(\delta t_{; q}^{v q}+\delta t_{; \chi}^{v \chi}+\delta t_{; \nu}^{\nu v}\right)\right]\right\} .
$$


It gives

$$
\begin{aligned}
& \ell(\ell+1) \frac{1}{q} \frac{d R_{\ell}^{m}}{d q}=-\rho_{0}\left(\eta_{1 \ell}^{W_{\ell}^{m}}+\eta_{2}^{W_{\ell}^{m}}\right)+\left(-3+2 \frac{h_{2}}{q}\right) \ell(\ell+1) \frac{R_{\ell}^{m}}{q^{2}} \\
& +\ell(\ell+1)\left\{[\ell(\ell+1)-2] \frac{\mu_{0}}{q}-2 \frac{h_{2}}{q} \frac{d \mu_{0}}{d q}\right\} \frac{W_{\ell}^{m}}{q^{2}}+3 m^{2} \frac{h_{2}}{q^{3}} \mu_{0} \frac{d W_{\ell}^{m}}{d q} \\
& +\sum_{\ell^{\prime}=|\ell-2|}^{\ell+2}\left[\begin{array}{ccc}
\ell & 2 & \ell^{\prime} \\
0 & 0 & 0 \\
m & 0 & m
\end{array}\right]\left\{\left(-2 \frac{h_{2}}{q^{2}}+\frac{3}{q} \frac{d h_{2}}{d q}+\frac{d^{2} h_{2}}{d q^{2}}\right) \frac{\ell^{\prime}\left(\ell^{\prime}+1\right)}{q}\left\{\begin{array}{c}
R_{\ell^{\prime}}^{m} \\
0
\end{array}\right\}\right. \\
& +\left[18 \frac{h_{2}}{q}+6 \frac{d h_{2}}{d q}+\ell^{\prime}\left(\ell^{\prime}+1\right)\left(\frac{h_{2}}{q}+2 \frac{d h_{2}}{d q}\right)\right] \frac{1}{q}\left\{\begin{array}{c}
\frac{d R_{\ell^{\prime}}^{m}}{d q} \\
0
\end{array}\right\} \\
& +\ell^{\prime}\left(\ell^{\prime}+1\right)\left\{2 \frac{d \mu_{0}}{d q}-3\left[\ell^{\prime}\left(\ell^{\prime}+1\right)+2\right] \frac{\mu_{0}}{q}\right\} \frac{h_{2}}{q^{3}}\left\{\begin{array}{c}
W_{\ell^{\prime}}^{m} \\
0
\end{array}\right\} \\
& \left.+6 \frac{h_{2}}{q}\left\{\begin{array}{c}
\frac{d^{2} R_{\ell^{\prime}}^{m}}{d q^{2}} \\
0
\end{array}\right\}+\left[\ell^{\prime}\left(\ell^{\prime}+1\right)-4\right] 6 \mu_{0} \frac{h_{2}}{q^{3}}\left\{\begin{array}{c}
\frac{d W_{\ell^{\prime}}^{m}}{d q} \\
0
\end{array}\right\}\right\} \\
& +\sum_{\ell^{\prime}=|\ell-2|}^{\ell+2}\left[\begin{array}{ccc}
\ell & 2 & \ell^{\prime} \\
0 & 1 & -1 \\
m & 0 & m
\end{array}\right] 2 \sqrt{3} L_{0}^{\ell^{\prime}} \\
& \times\left\{\left[(4-\ell(\ell+1)) \frac{h_{2}}{q^{2}}+\frac{3}{q} \frac{d h_{2}}{d q}+\frac{d^{2} h_{2}}{d q^{2}}\right]\left\{\begin{array}{c}
\frac{R_{\ell^{\prime}}^{m}}{q} \\
0
\end{array}\right\}+\left(4 \frac{h_{2}}{q^{2}}+\frac{3}{q} \frac{d h_{2}}{d q}\right)\left\{\begin{array}{c}
\frac{d R_{\ell^{\prime}}^{m}}{d q} \\
0
\end{array}\right\}+\frac{h_{2}}{q}\left\{\begin{array}{c}
\frac{d^{2} R_{\ell^{\prime}}^{m}}{d q^{2}} \\
i \frac{d^{2} Q_{\ell^{\prime}}^{m}}{d q^{2}}
\end{array}\right\}\right. \\
& -\left[\ell^{\prime}\left(\ell^{\prime}+1\right) \frac{h_{2}}{q^{2}}+\frac{3}{q} \frac{d h_{2}}{d q}+\frac{d^{2} h_{2}}{d q^{2}}\right]\left\{\begin{array}{c}
0 \\
i \frac{Q_{\ell^{\prime}}^{m}}{q}
\end{array}\right\}+\left(2 \frac{h_{2}}{q^{2}}-\frac{1}{q} \frac{d h_{2}}{d q}\right)\left\{\begin{array}{c}
0 \\
i \frac{d Q_{\ell^{\prime}}^{m}}{d q}
\end{array}\right\} \\
& +\left[6 \beta_{0}-\ell^{\prime}\left(\ell^{\prime}+1\right) \mu_{0}\right] \frac{h_{2}}{q^{4}}\left\{\begin{array}{c}
0 \\
i U_{\ell^{\prime}}^{m}
\end{array}\right\}+\left(2 \frac{\lambda_{0}}{q}-\frac{d \lambda_{0}}{d q}\right) \frac{h_{2}}{q^{2}}\left\{\begin{array}{c}
0 \\
i \frac{d U_{\ell^{\prime}}^{m}}{d q}
\end{array}\right\}-\lambda_{0} \frac{h_{2}}{q^{2}}\left\{\begin{array}{c}
0 \\
i \frac{d^{2} U_{\ell^{\prime}}^{m}}{d q^{2}}
\end{array}\right\} \\
& +\ell^{\prime}\left(\ell^{\prime}+1\right)\left(\frac{d \lambda_{0}}{d q}-3 \frac{\beta_{0}}{q}\right) \frac{h_{2}}{q^{3}}\left\{\begin{array}{c}
0 \\
i V_{\ell^{\prime}}^{m}
\end{array}\right\}+\left[4 \mu_{0}+\ell^{\prime}\left(\ell^{\prime}+1\right)\left(\lambda_{0}+\mu_{0}\right)\right] \frac{h_{2}}{q^{3}}\left\{\begin{array}{c}
0 \\
i \frac{d V_{\ell^{\prime}}^{m}}{d q}
\end{array}\right\} \\
& \left.+\left\{\left[2-\ell^{\prime}\left(\ell^{\prime}+1\right)\right] \frac{d \mu_{0}}{d q}-\left[4+7 \ell^{\prime}\left(\ell^{\prime}+1\right)\right] \frac{\mu_{0}}{q}\right\} \frac{h_{2}}{q^{3}}\left\{\begin{array}{c}
W_{\ell^{\prime}}^{m} \\
0
\end{array}\right\}+\left[19+\ell^{\prime}\left(\ell^{\prime}+1\right)-3 \ell(\ell+1)\right] \mu_{0} \frac{h_{2}}{q^{3}}\left\{\begin{array}{c}
\frac{d W_{\ell^{\prime}}^{m}}{d q} \\
0
\end{array}\right\}\right\} \\
& +\sum_{\ell^{\prime}=|\ell-1|}^{\ell+1}\left(2 L_{0}^{\ell^{\prime}}\left[\begin{array}{ccc}
\ell & 1 & \ell^{\prime} \\
0 & 1 & -1 \\
m & 0 & m
\end{array}\right]-\left[\begin{array}{ccc}
\ell & 1 & \ell^{\prime} \\
0 & 0 & 0 \\
m & 0 & m
\end{array}\right]\right) 12 m L_{0}^{\ell^{\prime}} \frac{h_{2}}{q^{3}} \frac{d \mu_{0}}{d q}\left\{\begin{array}{c}
0 \\
i V_{\ell^{\prime}}^{m}
\end{array}\right\} \\
& +\sum_{\ell^{\prime}=|\ell-1|}^{\ell+1} \sum_{\ell^{\prime \prime}=\left|\ell^{\prime}-1\right|}^{\ell^{\prime}+1}\left[\begin{array}{ccc}
\ell^{\prime} & 1 & \ell^{\prime \prime} \\
0 & 1 & -1 \\
m & 0 & m
\end{array}\right]\left(2 L_{0}^{\ell^{\prime}}\left[\begin{array}{ccc}
\ell & 1 & \ell^{\prime} \\
0 & 1 & -1 \\
m & 0 & m
\end{array}\right]-\left[\begin{array}{ccc}
\ell & 1 & \ell^{\prime} \\
0 & 0 & 0 \\
m & 0 & m
\end{array}\right]\right) 6 \frac{h_{2}}{q^{3}} L_{0}^{\ell^{\prime \prime}}\left\{2 R_{\ell^{\prime \prime}}^{m}+\mu_{0} \frac{d W_{\ell^{\prime \prime}}^{m}}{d q}-2 \frac{d \mu_{0}}{d q} W_{\ell^{\prime \prime}}^{m}\right\} \text {, }
\end{aligned}
$$

where

$$
\begin{aligned}
\eta_{1 \ell}^{W^{m}}= & \frac{\omega^{2}}{q}\left\{\ell(\ell+1) W_{\ell}^{m}+\sum_{\ell^{\prime}=|\ell-2|}^{\ell+2}\left[\begin{array}{ccc}
\ell & 2 & \ell^{\prime} \\
0 & 0 & 0 \\
m & 0 & m
\end{array}\right] h_{2}\left\{-6 \frac{d W_{\ell^{\prime}}^{m}}{d q}-\ell^{\prime}\left(\ell^{\prime}+1\right) \frac{W_{\ell^{\prime}}^{m}}{q}\right\}\right. \\
& \left.\left.\left.+\left[\begin{array}{ccc}
\ell & 2 & \ell^{\prime} \\
0 & 1 & -1 \\
m & 0 & m
\end{array}\right] 2 \sqrt{3} h_{2} L_{0}^{\ell^{\prime}}\left\{\begin{array}{c}
\frac{W_{\ell^{\prime}}^{m}}{q}-\frac{d W_{\ell^{\prime}}^{m}}{d q} \\
V_{\ell^{\prime}}^{m} \\
q
\end{array}+\frac{d V_{\ell^{\prime}}^{m}}{d q}\right)\right\}\right]\right\} \\
+ & \frac{2}{q} \omega \Omega_{0}\left\{\sum _ { \ell ^ { \prime } = | \ell - 1 | } ^ { \ell + 1 } \left[\left[\begin{array}{ccc}
\ell & 1 & \ell^{\prime} \\
-1 & 0 & -1 \\
m & 0 & m
\end{array}\right] 2 L_{0}^{\ell} L_{0}^{\ell^{\prime}}\left\{\begin{array}{c}
W_{\ell^{\prime}}^{m} \\
i V_{\ell^{\prime}}^{m}
\end{array}\right\}+\left[\begin{array}{ccc}
\ell & 1 & \ell^{\prime} \\
-1 & -1 & 0 \\
m & 0 & m
\end{array}\right] L_{0}^{\ell}\left\{\begin{array}{c}
0 \\
-2 i U_{\ell^{\prime}}^{m}
\end{array}\right\}\right.\right.
\end{aligned}
$$




$$
\begin{aligned}
& +\left[\begin{array}{ccc}
\ell & 1 & \ell^{\prime} \\
0 & 0 & 0 \\
m & 0 & m
\end{array}\right] \frac{6}{5} h_{2}\left\{i\left(\frac{d V_{\ell^{\prime}}^{m}}{d q}-\frac{U_{\ell^{\prime}}^{m}}{q}\right)\right\} \\
& +\left[\begin{array}{ccc}
\ell & 1 & \ell^{\prime} \\
0 & 1 & -1 \\
m & 0 & m
\end{array}\right] 2 h_{2} L_{0}^{\ell^{\prime}}\left\{\begin{array}{c}
\frac{d W_{\ell^{\prime}}^{m}}{d q}-\frac{W_{\ell^{\prime}}^{m}}{q} \\
\left.-i\left(\frac{V_{\ell^{\prime}}^{m}}{q}+\frac{d V_{\ell^{\prime}}^{m}}{d q}\right)\right\}
\end{array}\right\} \\
& +\sum_{\ell^{\prime}=|\ell-3|}^{\ell+3}\left[\begin{array}{ccc}
\ell & 3 & \ell^{\prime} \\
0 & 0 & 0 \\
m & 0 & m
\end{array}\right] \frac{6}{5} h_{2}\left\{i\left(\frac{U_{\ell^{\prime}}^{m}}{q}-4 \frac{d V_{\ell^{\prime}}^{m}}{d q}\right)\right\} \\
& +\sum_{\ell^{\prime}=|\ell-2| \ell^{\prime \prime}=\left|\ell^{\prime}-1\right|}^{\ell+2} \sum_{\ell^{\prime}+1}\left[\begin{array}{ccc}
\ell & 2 & \ell^{\prime} \\
0 & 0 & 0 \\
m & 0 & m
\end{array}\right]\left[\begin{array}{ccc}
\ell^{\prime} & 1 & \ell^{\prime \prime} \\
-1 & 0 & -1 \\
m & 0 & m
\end{array}\right] \frac{h_{2}}{q} 2 L_{0}^{\ell^{\prime}} L_{0}^{\ell^{\prime \prime}}\left\{\begin{array}{l}
-W_{\ell^{\prime \prime}}^{m} \\
-i V_{\ell^{\prime \prime}}^{m}
\end{array}\right\} \\
& +\sum_{\ell^{\prime}=|\ell-2| \ell^{\prime \prime}=\left|\ell^{\prime}-1\right|}^{\ell+2} \sum_{\ell^{\prime}+1}\left[\begin{array}{ccc}
\ell & 2 & \ell^{\prime} \\
0 & 0 & 0 \\
m & 0 & m
\end{array}\right]\left[\begin{array}{ccc}
\ell^{\prime} & 1 & \ell^{\prime \prime} \\
-1 & -1 & 0 \\
m & 0 & m
\end{array}\right] \frac{h_{2}}{q} L_{0}^{\ell^{\prime}}\left\{\begin{array}{c}
0 \\
i 2 U_{\ell^{\prime \prime}}^{m}
\end{array}\right\} \\
& \left.+\sum_{\ell^{\prime}=|\ell-2| \ell^{\prime \prime}=\left|\ell^{\prime}-1\right|}^{\ell+2} \sum_{\ell^{\prime}+1}\left[\begin{array}{ccc}
\ell & 2 & \ell^{\prime} \\
0 & 0 & 0 \\
m & 0 & m
\end{array}\right]\left[\begin{array}{ccc}
\ell^{\prime} & 1 & \ell^{\prime \prime} \\
0 & 1 & -1 \\
m & 0 & m
\end{array}\right] 4 h_{2} L_{0}^{\ell^{\prime \prime}}\left\{\begin{array}{c}
\frac{d W_{\ell^{\prime \prime}}^{m}}{d q}-\frac{W_{\ell^{\prime \prime}}^{m}}{q} \\
i\left(-\frac{V_{\ell^{\prime \prime}}^{m}}{q}-\frac{d V_{\ell^{\prime \prime}}^{m}}{d q}\right)
\end{array}\right\}\right\}
\end{aligned}
$$

and

$$
\begin{aligned}
& \eta_{2 \ell}^{W m}=12 \pi \mathrm{G} \rho_{0} m^{2} \frac{h_{2}^{2}}{q^{3}}\left(W_{\ell}^{m}+\sum_{\ell^{\prime}=|\ell-2|}^{\ell+2}\left[\begin{array}{ccc}
\ell & 2 & \ell^{\prime} \\
0 & 0 & 0 \\
m & 0 & m
\end{array}\right]\left\{\begin{array}{c}
2 W_{\ell^{\prime}}^{m} \\
0
\end{array}\right\}\right) \\
& +\sum_{\ell^{\prime}=|\ell-2|}^{\ell+2}\left[\begin{array}{ccc}
\ell & 2 & \ell^{\prime} \\
0 & 1 & -1 \\
m & 0 & m
\end{array}\right] 2 \sqrt{3} L_{0}^{\ell^{\prime}} \frac{h_{2}}{q^{2}}\left\{i\left(2 \frac{\phi_{\ell^{\prime}}^{m}}{q}-g_{\ell^{\prime}}^{m}+4 \pi \mathrm{G} \rho_{0} U_{\ell^{\prime}}^{m}\right)\right\} \\
& +\sum_{\ell^{\prime}=|\ell-4|}^{\ell+4}\left[\begin{array}{ccc}
\ell & 4 & \ell^{\prime} \\
0 & 1 & -1 \\
m & 0 & m
\end{array}\right] 2 \sqrt{10} L_{0}^{\ell^{\prime}} \frac{h_{4}}{q^{2}}\left\{i\left(2 \frac{\phi_{\ell^{\prime}}^{m}}{q}-g_{\ell^{\prime}}^{m}+4 \pi \mathrm{G} \rho_{0} U_{\ell^{\prime}}^{m}\right)\right\}
\end{aligned}
$$

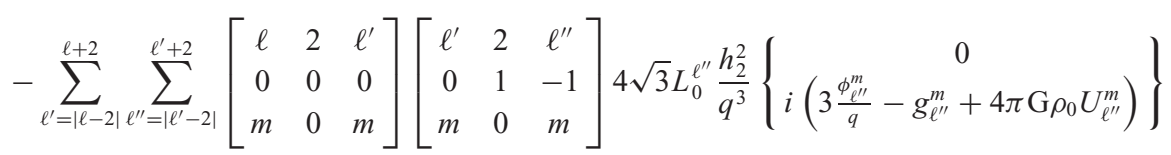

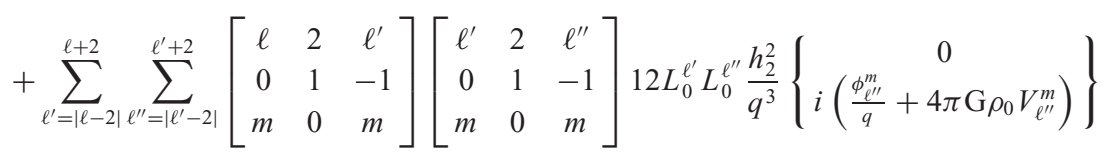

$$
\begin{aligned}
& +\frac{d \tilde{\phi}_{0}}{d q}\left\{3 m^{2}\left[\frac{h_{2}}{q}\left(3 \frac{h_{2}}{q}-\frac{d h_{2}}{d q}\right) \frac{W_{\ell}^{m}}{q^{2}}-\frac{h_{2}^{2}}{q^{3}} \frac{d W_{\ell}^{m}}{d q}\right]\right. \\
& +\sum_{\ell^{\prime}=|\ell-2|}^{\ell+2}\left[\begin{array}{ccc}
\ell & 2 & \ell^{\prime} \\
0 & 0 & 0 \\
m & 0 & m
\end{array}\right] 6 m^{2}\left[\frac{h_{2}}{q^{3}}\left(3 \frac{h_{2}}{q}-\frac{d h_{2}}{d q}\right)\left\{\begin{array}{c}
W_{\ell^{\prime}}^{m} \\
0
\end{array}\right\}-\frac{h_{2}^{2}}{q^{3}}\left\{\begin{array}{c}
\frac{d W_{\ell^{\prime}}^{m}}{d q} \\
0
\end{array}\right\}\right] \\
& +\sum_{\ell^{\prime}=|\ell-2|}^{\ell+2}\left[\begin{array}{ccc}
\ell & 2 & \ell^{\prime} \\
0 & 1 & -1 \\
m & 0 & m
\end{array}\right] 2 \sqrt{3} L_{0}^{\ell^{\prime}}\left[-4 \frac{h_{2}}{q^{3}}\left\{\begin{array}{c}
0 \\
i U_{\ell^{\prime}}^{m}
\end{array}\right\}+\left(\frac{h_{2}}{q}-\frac{d h_{2}}{d q}\right) \frac{1}{q}\left\{\begin{array}{c}
0 \\
i \frac{d U_{\ell \ell^{\prime}}^{m}}{d q}
\end{array}\right\}+\ell^{\prime}\left(\ell^{\prime}+1\right) \frac{h_{2}}{q^{2}}\left\{\begin{array}{c}
0 \\
i \frac{d V_{\ell \ell^{\prime}}^{m}}{d q}
\end{array}\right\}\right] \\
& +\sum_{\ell^{\prime}=|\ell-4|}^{\ell+4}\left[\begin{array}{ccc}
\ell & 4 & \ell^{\prime} \\
0 & 1 & -1 \\
m & 0 & m
\end{array}\right] 2 \sqrt{10} L_{0}^{\ell^{\prime}}\left[-4 \frac{h_{4}}{q^{3}}\left\{\begin{array}{c}
0 \\
i U_{\ell^{\prime}}^{m}
\end{array}\right\}+\left(\frac{h_{4}}{q}-\frac{d h_{4}}{d q}\right) \frac{1}{q}\left\{\begin{array}{c}
0 \\
i \frac{d U_{\ell^{\prime}}^{m}}{d q}
\end{array}\right\}+\ell^{\prime}\left(\ell^{\prime}+1\right) \frac{h_{4}}{q^{2}}\left\{\begin{array}{c}
0 \\
i \frac{d V_{\ell^{\prime}}^{m}}{d q}
\end{array}\right\}\right]
\end{aligned}
$$




$$
\begin{aligned}
& +\sum_{\ell^{\prime}=|\ell-2| \ell^{\prime \prime}=\left|\ell^{\prime}-2\right|}^{\ell+2} \sum^{\ell^{\prime}+2}\left[\begin{array}{ccc}
\ell & 2 & \ell^{\prime} \\
0 & 0 & 0 \\
m & 0 & m
\end{array}\right]\left[\begin{array}{ccc}
\ell^{\prime} & 2 & \ell^{\prime \prime} \\
0 & 1 & -1 \\
m & 0 & m
\end{array}\right] 2 \sqrt{3} L_{0}^{\ell^{\prime \prime}} \\
& \times\left\{\frac{h_{2}}{q^{2}}\left(-\frac{d^{2} h_{2}}{d q^{2}}-\frac{2}{q} \frac{d h_{2}}{d q}+8 \frac{h_{2}}{q^{2}}\right)\left\{\begin{array}{c}
0 \\
i U_{\ell^{\prime \prime}}^{m}
\end{array}\right\}-\frac{h_{2}}{q^{2}}\left(2 \frac{h_{2}}{q}+\frac{d h_{2}}{d q}\right)\left\{\begin{array}{c}
0 \\
i \frac{d U_{\ell^{\prime \prime}}^{m}}{d q}
\end{array}\right\}-\frac{h_{2}^{2}}{q^{3}}\left\{i\left[\ell^{\prime \prime}\left(\ell^{\prime \prime}+1\right) \frac{V_{\ell^{\prime \prime}}^{m}}{q}+6 \frac{d V_{\ell^{\prime \prime}}^{m}}{d q}\right]\right\}\right\} \\
& +\sum_{\ell^{\prime}=|\ell-2| \ell^{\prime \prime}=\left|\ell^{\prime}-2\right|}^{\ell+2} \sum^{\ell^{\prime}+2}\left[\begin{array}{ccc}
\ell & 2 & \ell^{\prime} \\
0 & 1 & -1 \\
m & 0 & m
\end{array}\right]\left[\begin{array}{ccc}
\ell^{\prime} & 2 & \ell^{\prime \prime} \\
0 & 1 & -1 \\
m & 0 & m
\end{array}\right] 12 L_{0}^{\ell} L_{0}^{\ell^{\prime \prime}} \frac{h_{2}}{q^{2}} \\
& \left.\times\left[\left(\frac{d h_{2}}{d q}-3 \frac{h_{2}}{q}\right)\left\{\begin{array}{c}
0 \\
i \frac{V_{\ell^{\prime \prime}}^{m}}{q}
\end{array}\right\}-3 \frac{h_{2}}{q}\left\{\begin{array}{c}
0 \\
i \frac{d V_{\ell^{\prime \prime}}^{m}}{d q}
\end{array}\right\}\right]\right\} \\
& +\frac{d^{2} \tilde{\phi}_{0}}{d q^{2}}\left\{3 m^{2} \frac{h_{2}^{2}}{q^{3}}\left(W_{\ell}^{m}+\sum_{\ell^{\prime}=|\ell-2|}^{\ell+2}\left[\begin{array}{ccc}
\ell & 2 & \ell^{\prime} \\
0 & 0 & 0 \\
m & 0 & m
\end{array}\right]\left\{\begin{array}{c}
2 W_{\ell^{\prime}}^{m} \\
0
\end{array}\right\}\right)\right. \\
& -\sum_{\ell^{\prime}=|\ell-2|}^{\ell+2}\left[\begin{array}{ccc}
\ell & 2 & \ell^{\prime} \\
0 & 1 & -1 \\
m & 0 & m
\end{array}\right] 2 \sqrt{3} L_{0}^{\ell^{\prime}} \frac{h_{2}}{q^{2}}\left\{\begin{array}{c}
0 \\
i U_{\ell^{\prime}}^{m}
\end{array}\right\} \\
& -\sum_{\ell^{\prime}=|\ell-4|}^{\ell+4}\left[\begin{array}{ccc}
\ell & 4 & \ell^{\prime} \\
0 & 1 & -1 \\
m & 0 & m
\end{array}\right] 2 \sqrt{10} i L_{0}^{\ell^{\prime}} \frac{h_{4}}{q^{2}}\left\{\begin{array}{c}
0 \\
i U_{\ell^{\prime}}^{m}
\end{array}\right\} \\
& +\sum_{\ell^{\prime}=|\ell-2| \ell^{\prime \prime}=\left|\ell^{\prime}-2\right|}^{\ell+2} \sum_{\ell^{\prime}+2}\left[\begin{array}{ccc}
\ell & 2 & \ell^{\prime} \\
0 & 0 & 0 \\
m & 0 & m
\end{array}\right]\left[\begin{array}{ccc}
\ell^{\prime} & 2 & \ell^{\prime \prime} \\
0 & 1 & -1 \\
m & 0 & m
\end{array}\right] 4 \sqrt{3} i L_{0}^{\ell^{\prime \prime}} \frac{h_{2}}{q^{2}}\left(\frac{h_{2}}{q}+\frac{d h_{2}}{d q}\right)\left\{\begin{array}{c}
0 \\
i U_{\ell^{\prime \prime}}^{m}
\end{array}\right\} \\
& \left.-\sum_{\ell^{\prime}=|\ell-2|}^{\ell+2} \sum_{\ell^{\prime \prime}=\left|\ell^{\prime}-2\right|}^{\ell^{\prime}+2}\left[\begin{array}{ccc}
\ell & 2 & \ell^{\prime} \\
0 & 1 & -1 \\
m & 0 & m
\end{array}\right]\left[\begin{array}{ccc}
\ell^{\prime} & 2 & \ell^{\prime \prime} \\
0 & 1 & -1 \\
m & 0 & m
\end{array}\right] 12 i L_{0}^{\ell} L_{0}^{\ell^{\prime \prime}} \frac{h_{2}^{2}}{q^{3}}\left\{\begin{array}{c}
0 \\
i V_{\ell^{\prime \prime}}^{m}
\end{array}\right\}\right\} \text {. }
\end{aligned}
$$

The harmonic components $U^{m}{ }_{\ell}, V^{m}{ }_{\ell}, W^{m}{ }_{\ell}, \phi^{m}{ }_{\ell}, g^{m}{ }_{\ell}, P^{m}{ }_{\ell}, Q^{m}{ }_{\ell}$, and $R^{m}{ }_{\ell}$ that satisfy the first-order ordinary differential equations (116), (118), (119), (122), (123), (128), (130), and (133) couple following the rules (97) or (98). To solve the system of ordinary differential equations numerically, the series (97) or (98) must be truncated at a finite level. If we denote by $\mathbf{y}$ the finite-dimensional vector whose components are the harmonic components of the spheroidal and toroidal vectors retained in the truncated series, the differential system can be written

$\frac{d \mathbf{y}}{d q}=\boldsymbol{A}^{\mathrm{s}} \mathbf{y}$

where $\boldsymbol{A}^{\mathrm{s}}$ is a square matrix of the same dimension as $\mathbf{y}$. The differential system (136) is suitable for numerical integration using standard finite-difference methods.

The numerical solution of the equations of motion of a realistic earth model, including fluid layers, will be reported in a later paper.

\subsection{Continuity conditions}

At any boundary between two continuous media, the following quantities must be continuous:

$\mathbf{s} \cdot \mathbf{n}$

$\mathbf{n} \cdot \delta \boldsymbol{t}=\delta \boldsymbol{t} \cdot \mathbf{n}$

$\Delta \phi$

$\Delta g$ 
Therefore, the following functions of $q$ must be continuous:

$$
\begin{aligned}
& U_{\ell}^{m}-\sum_{\ell^{\prime}=|\ell-2|}^{\ell+2}\left[\begin{array}{ccc}
\ell & 2 & \ell^{\prime} \\
0 & 1 & -1 \\
m & 0 & m
\end{array}\right] 2 \sqrt{3} L_{0}^{\ell^{\prime}} \frac{h_{2}}{q}\left\{\begin{array}{c}
-V_{\ell^{\prime}}^{m} \\
i W_{\ell^{\prime}}^{m}
\end{array}\right\} \\
& P_{\ell}^{m}-\sum_{\ell^{\prime}=|\ell-2|}^{\ell+2}\left[\begin{array}{ccc}
\ell & 2 & \ell^{\prime} \\
0 & 1 & -1 \\
m & 0 & m
\end{array}\right] 2 \sqrt{3} L_{0}^{\ell^{\prime}} \frac{h_{2}}{q}\left\{\begin{array}{c}
-Q_{\ell^{\prime}}^{m} \\
i R_{\ell^{\prime}}^{m}
\end{array}\right\} \\
& \ell(\ell+1) Q_{\ell}^{m}+\left[2 \ell(\ell+1)-6 m^{2}\right] \frac{h_{2}}{q} \mu_{0} \frac{V_{\ell}^{m}}{q} \\
& +\sum_{\ell^{\prime}=|\ell-2|}^{\ell+2}\left[\begin{array}{ccc}
\ell & 2 & \ell^{\prime} \\
0 & 0 & 0 \\
m & 0 & m
\end{array}\right]\left[-\frac{d h_{2}}{d q}\left\{\begin{array}{c}
\ell^{\prime}\left(\ell^{\prime}+1\right) Q_{\ell^{\prime}}^{m} \\
0
\end{array}\right\}\right. \\
& \left.+6 \frac{h_{2}}{q}\left\{\begin{array}{c}
P_{\ell^{\prime}}^{m}-q \frac{d Q_{\ell^{\prime}}^{m}}{d q}-2\left(\lambda_{0}+\mu_{0}\right) \frac{U_{\ell^{\prime}}^{m}}{q}-\lambda_{0} \frac{d U_{\ell^{\prime}}^{m}}{d q}+\ell^{\prime}\left(\ell^{\prime}+1\right)\left(\lambda_{0}+\frac{4}{3} \mu_{0}\right) \frac{V_{\ell^{\prime}}^{m}}{q} \\
0
\end{array}\right\}\right] \\
& +\sum_{\ell^{\prime}=|\ell-2|}^{\ell+2}\left[\begin{array}{ccc}
\ell & 2 & \ell^{\prime} \\
0 & 1 & -1 \\
m & 0 & m
\end{array}\right] 2 \sqrt{3} L_{0}^{\ell^{\prime}}\left[\frac{d h_{2}}{d q}\left\{\begin{array}{c}
-Q_{\ell^{\prime}}^{m} \\
i R_{\ell^{\prime}}^{m}
\end{array}\right\}-\frac{h_{2}}{q}\left\{\begin{array}{c}
-P_{\ell^{\prime}}^{m}+q \frac{d Q_{\ell^{\prime}}^{m}}{d q}+2\left(\lambda_{0}+\mu_{0}\right) \frac{U_{\ell^{\prime}}^{m}}{q}+\lambda_{0} \frac{d U_{\ell^{\prime}}^{m}}{d q} \\
0
\end{array}\right\}\right. \\
& \left.-\frac{h_{2}}{q}\left\{\begin{array}{c}
{\left[-\ell^{\prime}\left(\ell^{\prime}+1\right) \lambda_{0}+(8-2 \ell(\ell+1)) \mu_{0}\right] \frac{V_{\ell^{\prime}}^{m}}{q}} \\
i\left[-\ell^{\prime}\left(\ell^{\prime}+1\right)-8+2 \ell(\ell+1)\right] \mu_{0} \frac{W_{\ell^{\prime}}^{m}}{q}
\end{array}\right\}\right] \\
& -\sum_{\ell^{\prime}=|\ell-2| \ell^{\prime \prime}=\left|\ell^{\prime}-1\right|}^{\ell+2}\left[\begin{array}{ccc}
\ell & 2 & \ell^{\prime} \\
0 & 0 & 0 \\
m & 0 & m
\end{array}\right]\left[\begin{array}{ccc}
\ell^{\prime} & 1 & \ell^{\prime \prime} \\
0 & 1 & -1 \\
m & 0 & m
\end{array}\right] 12 L_{0}^{\ell^{\prime \prime}} m \frac{h_{2}}{q} \mu_{0}\left\{\begin{array}{c}
0 \\
i \frac{W_{\ell^{\prime \prime}}^{m}}{q}
\end{array}\right\} \\
& \ell(\ell+1) R_{\ell}^{m}+\sum_{\ell^{\prime}=|\ell-2|}^{\ell+2}\left[\begin{array}{ccc}
\ell & 2 & \ell^{\prime} \\
0 & 0 & 0 \\
m & 0 & m
\end{array}\right]\left[-\frac{d h_{2}}{d q}\left\{\begin{array}{c}
\ell^{\prime}\left(\ell^{\prime}+1\right) Q_{\ell^{\prime}}^{m} \\
0
\end{array}\right\}-6 \frac{h_{2}}{q}\left\{\begin{array}{c}
q \frac{d Q_{\ell^{\prime}}^{m}}{d q}-\ell^{\prime}\left(\ell^{\prime}+1\right) \mu_{0} \frac{W_{\ell^{\prime}}^{m}}{q} \\
0
\end{array}\right\}\right]
\end{aligned}
$$

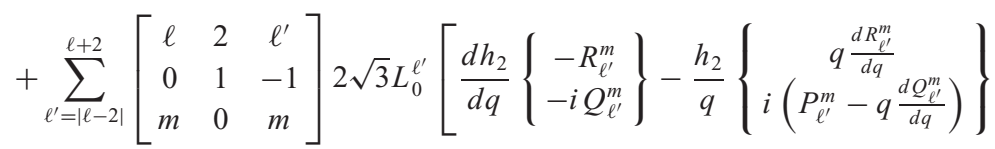

$$
\begin{aligned}
& \left.+\frac{h_{2}}{q}\left\{i\left[2\left(\lambda_{0}+\mu_{0}\right) \frac{U_{\ell^{\prime}}^{m}}{q}+\lambda_{0} \frac{d U_{\ell^{\prime}}^{m}}{d q}+\left[-\ell^{\prime}\left(\ell^{\prime}+1\right) \lambda_{0}+2 \mu_{0}\right] \frac{V_{\ell^{\prime}}^{m}}{q}\right]\right\}\right] \\
& -\sum_{\ell^{\prime}=|\ell-2|}^{\ell+2} \sum_{\ell^{\prime \prime}=\left|\ell^{\prime}-1\right|}^{\ell^{\prime}+1}\left[\begin{array}{ccc}
\ell & 2 & \ell^{\prime} \\
0 & 0 & 0 \\
m & 0 & m
\end{array}\right]\left[\begin{array}{ccc}
\ell^{\prime} & 1 & \ell^{\prime \prime} \\
0 & 1 & -1 \\
m & 0 & m
\end{array}\right] 12 L_{0}^{\ell^{\prime \prime}} m \frac{h_{2}}{q} \mu_{0}\left\{\begin{array}{c}
0 \\
i \frac{V_{\ell^{\prime \prime}}^{m}}{q}
\end{array}\right\}
\end{aligned}
$$

$g_{\ell}^{m}$

If the boundary is welded, $\mathbf{s}$ is continuous. Therefore, instead of quantity (141), the quantities

$$
\left.\begin{array}{l}
U_{\ell}^{m}+\sum_{\ell^{\prime}=|\ell-2|}^{\ell+2}\left[\begin{array}{ccc}
\ell & 2 & \ell^{\prime} \\
0 & 0 & 0 \\
m & 0 & m
\end{array}\right] \frac{d h_{2}}{d q}\left\{\begin{array}{c}
U_{\ell^{\prime}}^{m} \\
0
\end{array}\right\} \\
\ell(\ell+1) V_{\ell}^{m}+\sum_{\ell^{\prime}=|\ell-2|}^{\ell+2}\left[\begin{array}{ccc}
\ell & 2 & \ell^{\prime} \\
0 & 0 & 0 \\
m & 0 & m
\end{array}\right] 6 h_{2}\left\{\frac{U_{\ell^{\prime}}^{m}}{q}-\frac{d V_{\ell^{\prime}}^{m}}{d q}\right. \\
0
\end{array}\right\}-\sum_{\ell^{\prime}=|\ell-2|}^{\ell+2}\left[\begin{array}{ccc}
\ell & 2 & \ell^{\prime} \\
0 & 1 & -1 \\
m & 0 & m
\end{array}\right] h_{2}\left\{\begin{array}{c}
\left(\frac{U_{\ell^{\prime}}^{m}}{q}-\frac{d V_{\ell^{\prime}}^{m}}{d q}\right) \\
i \frac{d W_{\ell^{\prime}}^{m}}{d q}
\end{array}\right\}
$$




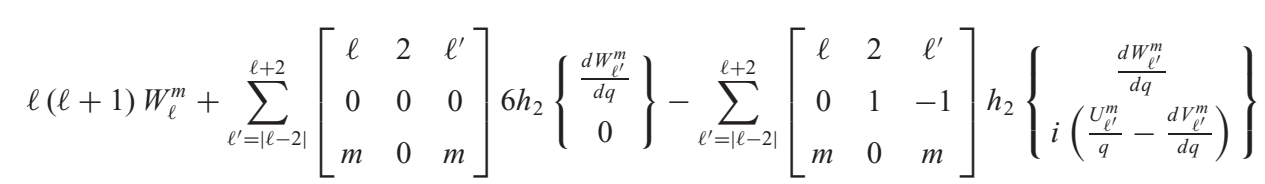

are continuous at a welded boundary.

\section{CONCLUSIONS}

We have introduced a normal-mode theory of a rotating hydrostatic earth model based both on a Lagrangian perturbation of a spherical reference model by rotation and on a Lagrangian perturbation of the rotating model. Denoting by $h$ the small discrepancy between the rotating earth model and the spherical model of reference, we have determined what terms in the equations of motion of the rotating model must be computed to the second order in $h$ in order to describe properly the nutational motions. $h$ is given as a function of the radius $q$ and the colatitude $\chi$ by the theory of hydrostatic equilibrium figures. To describe the seismic normal modes, a first-order approximation is sufficient. We have expanded the equations of motion in surface spherical harmonics to obtain ordinary differential equations over radius $q$.

We have limited our theory throughout to hydrostatic earth models. Nevertheless, if we neglect the unknown initial deviatoric stress, the theory can be applied to non-hydrostatic earth models by including the non-hydrostatic structure in $h$. Part of the theory should then be extended to take into account aspherical structure of harmonic degrees different from 2 and 4.

\section{A CKNOWLEDGMENTS}

This research was initiated while YR held a Postdoctoral Fellowship supported by the Natural Sciences and Engineering Research Council of Canada research grant no. A-1182 awarded to MGR. It was completed while YR held a CNAP Associate Physicist position at EOST. YR also acknowledges support from a CNRS-INSU grant (DyETI).

We thank an anonymous reviewer for pointing out an error in the original manuscript.

\section{REFERENCES}

Alterman, Z., Jarosch, H. \& Pekeris, L., 1959. Oscillations of the Earth, Proc. R. Soc. Lond., A, 252, 80-95.

Bryan, G.H., 1889. The waves on a rotating liquid spheroid of finite ellipticity, Phil. Trans. R. Soc. A, 180, 187-219.

Dahlen, F.A., 1968. The normal modes of a rotating, elliptical Earth, Geophys. J. R. astr. Soc., 16, 329-367.

Dahlen, F.A., 1972. Elastic dislocation theory of a self-gravitating elastic configuration with an initial static stress field, Geophys. J. R. astr. Soc., 28, 357-383.

Dahlen, F.A. \& Sailor, R.V., 1979. Rotational and elliptical splitting of the free oscillations of the Earth, Geophys. J. R. astr. Soc., 58, 609-623.

Denis C., 1989. The hydrostatic figure of the Earth, in Gravity and LowFrequency Geodynamics, pp. 111-186, ed. Teisseyre R., Physics and Evolution of the Earth's Interior, 4, Elsevier, Amsterdam.

Denis, C., Amalvict, M., Rogister, Y. \& Tomecka-Suchon, S., 1998. Methods for computing the internal flattening, with applications to the Earth's structure and geodynamics, Geophys. J. Int., 132, 603-642.

Dziewonski, A. \& Anderson, D., 1981. Preliminary reference Earth model, Phys. Earth planet. Int., 25, 297-356.

Dziewonski, A.M., Hales, A.L. \& Lapwood, E.R., 1975. Parametrically simple Earth models consistent with geophysical data, Phys. Earth planet. Inter., 10, 12-48.

Edmonds, A.R., 1960. Angular Momentum in Quantum Mechanics, Princeton University Press, Princeton, NJ.

Jeffreys, H., 1942. A derivation of the tidal equations, Proc. R. Soc. Lond., A, 181, 20-22.

Mathews, P.M., Buffett, B.A., Herring, T.A. \& Shapiro, I.I., 1991a. Forced nutations of the Earth: Influence of inner core dynamics, 1. Theory, J. geophys. Res., 96, 8219-8242.

Mathews, P.M., Buffett, B.A., Herring, T.A. \& Shapiro, I.I., 1991b. Forced nutations of the Earth: Influence of inner core dynamics, 2. Numerical results and comparisons, J. geophys. Res., 96, 8243-8257.

Moritz, H. \& Mueller, I., 1987. Earth Rotation, The Ungar Publishing Company, New York.

Robin, L., 1957. Fonctions de Legendre Sphériques et Fonctions Sphéroïdales, Gauthier-Villars, Paris.

Rogister, Y., 2001. On the diurnal and nearly diurnal free modes of the Earth, Geophys. J. Int., 144, 459-470.

Rogister, Y., 2003. Splitting of seismic free oscillations and of the Slichter triplet using the normal mode theory of a rotating, ellipsoidal Earth, Phys. Earth planet. Int., 140, 169-182.

Schastok, J., 1997. A new nutation series for a more realistic model Earth, Geophys. J. Int., 130, 137-150.

Smith, M.L., 1974. The scalar equations of infinitesimal elastic-gravitational motion for a rotating, slightly elliptical Earth, Geophys. J. R. astr. Soc., 37, 491-526.

Smith, M.L., 1976. Translational inner core oscillations of a rotating, slightly elliptical Earth, J. geophys. Res., 81, 3055-3065.

Smith, M.L., 1977. Wobble and nutation of the Earth, Geophys. J. R. astr. Soc., 50, 103-140.

Synge, J.L. \& Schild, A., 1978. Tensor Calculus, Dover Publications, Inc., New York.

Usami, T. \& Sato, Y., 1962. Torsional oscillation of a homogeneous elastic spheroid, Bull. seism. Soc. Am., 52, 469-484.

Zharkov, V.N. \& Trubitsyn, V.P., 1978. Physics of Planetary Interiors, ed. Hubbard W.B., Pachart Publishing House, Tucson, AZ.

\section{APPENDIX A: SPHERICAL HARMONICS}

We give here the definition and, without proof, some properties of the surface spherical harmonics.

\section{A1 Definition}

In order to exploit properties enunciated by Smith (1974), the surface spherical harmonics $\mathcal{D}_{\ell}^{m}(\chi, v)$ that we use throughout this paper are a particular case of the generalized surface spherical harmonics used by Smith: 
$\mathcal{D}_{\ell}^{m}(\chi, v)=\sqrt{\frac{(\ell-m) !}{(\ell+m) !}} P_{\ell}^{m}(\cos \chi) e^{i m \nu}$,

where

$P_{\ell}^{m}(x)=\frac{1}{2^{\ell} \ell !} \sqrt{\left(1-x^{2}\right)^{m}} \frac{d^{\ell+m}}{d x^{\ell+m}}\left(x^{2}-1\right)^{\ell}$

is the associated Legendre function of degree $\ell$ and order $m$. In particular, we have

$\mathcal{D}_{\ell}^{0}(\chi)=P_{\ell}(\cos \chi)=P_{\ell}^{0}(\cos \chi)$,

where $P_{\ell}(x)$ is the Legendre polynomial of degree $\ell$. The function $P^{-m}{ }_{\ell}(x)$ is defined by

$P_{\ell}^{-m}(x)=\frac{(\ell-m) !}{(\ell+m) !} P_{\ell}^{m}(x)$.

The spherical harmonics are orthogonal but they are not normalized. Indeed, we have

$\int_{0}^{2 \pi} \int_{0}^{\pi} \mathcal{D}_{\ell}^{m}(\chi, v) \mathcal{D}_{\ell^{\prime}}^{m^{\prime *}}(\chi, v) \sin \chi d \chi d v=\frac{4 \pi}{2 \ell+1} \delta_{\ell \ell^{\prime}} \delta_{m m^{\prime}}$,

where $\delta_{\ell \ell^{\prime}}$ is the Kronecker delta symbol. The spherical harmonics satisfy the second-order partial differential equation

$\frac{\partial^{2} \mathcal{D}_{\ell}^{m}}{\partial \chi^{2}}+\frac{\cos \chi}{\sin \chi} \frac{\partial \mathcal{D}_{\ell}^{m}}{\partial \chi}+\frac{1}{\sin ^{2} \chi} \frac{\partial^{2} \mathcal{D}_{\ell}^{m}}{\partial v^{2}}=-\ell(\ell+1) \mathcal{D}_{\ell}^{m}$.

\section{A2 Recurrence relations}

The following recurrence relations can be derived easily from formulae whose proofs can be found, for example, in Robin 1957 (Vol. I, pp. 98-103):

$$
\begin{aligned}
& \sin \chi \frac{\partial \mathcal{D}_{\ell}^{m}}{\partial \chi}=\frac{\ell}{2 \ell+1} \sqrt{(\ell+m+1)(\ell-m+1)} \mathcal{D}_{\ell+1}^{m}-\frac{\ell+1}{2 \ell+1} \sqrt{(\ell+m)(\ell-m)} \mathcal{D}_{\ell-1}^{m}, \\
& \cos \chi \mathcal{D}_{\ell}^{m}=\frac{1}{2 \ell+1} \sqrt{(\ell+m+1)(\ell-m+1)} \mathcal{D}_{\ell+1}^{m}+\frac{1}{2 \ell+1} \sqrt{(\ell+m)(\ell-m)} \mathcal{D}_{\ell-1}^{m}, \\
& \sin ^{2} \chi \frac{\partial^{2} \mathcal{D}_{\ell}^{m}}{\partial \chi^{2}}=\frac{\ell^{2}}{(2 \ell+1)(2 \ell+3)} \sqrt{(\ell+m+1)(\ell-m+1)(\ell+m+2)(\ell-m+2)} \mathcal{D}_{\ell+2}^{m} \\
& +\frac{2 m^{2}\left(\ell^{2}+\ell-3\right)-\ell(\ell+1)\left(2 \ell^{2}+2 \ell-3\right)}{(2 \ell+3)(2 \ell-1)} \mathcal{D}_{\ell}^{m} \\
& +\frac{(\ell+1)^{2}}{(2 \ell+1)(2 \ell-1)} \sqrt{(\ell+m-1)(\ell-m-1)(\ell+m)(\ell-m)} \mathcal{D}_{\ell-2}^{m} .
\end{aligned}
$$

In particular, we have

$\frac{d}{d \chi} P_{2 \ell}^{0}(\cos \chi)=-P_{2 \ell}^{1}(\cos \chi)$.

\section{A3 Product of spherical harmonics}

The product of two spherical harmonics is given by

$\mathcal{D}_{\ell_{1}}^{m_{1}} \mathcal{D}_{\ell_{2}}^{m_{2}}=\sum_{\ell=\left|\ell_{2}-\ell_{1}\right|}^{\left|\ell_{2}+\ell_{1}\right|}\left[\begin{array}{ccc}\ell & \ell_{1} & \ell_{2} \\ 0 & 0 & 0 \\ m & m_{1} & m_{2}\end{array}\right] \mathcal{D}_{\ell}^{m}$

where $m=m_{1}+m_{2}$. Adopting the notation of Smith (1974), we use the J-square symbol

$\left[\begin{array}{ccc}\ell & \ell_{1} & \ell_{2} \\ 0 & 0 & 0 \\ m & m_{1} & m_{2}\end{array}\right]$

that involves the product of the Wigner 3-j symbols $\left(\begin{array}{ccc}\ell & \ell_{1} & \ell_{2} \\ 0 & 0 & 0\end{array}\right)$ and $\left(\begin{array}{ccc}\ell & \ell_{1} & \ell_{2} \\ -m & m_{1} & m_{2}\end{array}\right)$ (Edmonds 1960): 
$\left[\begin{array}{ccc}\ell & \ell_{1} & \ell_{2} \\ 0 & 0 & 0 \\ m & m_{1} & m_{2}\end{array}\right]=(-1)^{m}(2 \ell+1)\left(\begin{array}{ccc}\ell & \ell_{1} & \ell_{2} \\ 0 & 0 & 0\end{array}\right)\left(\begin{array}{ccc}\ell & \ell_{1} & \ell_{2} \\ -m & m_{1} & m_{2}\end{array}\right)$.

More generally, we have

$\left[\begin{array}{ccc}\ell & \ell_{1} & \ell_{2} \\ n & n_{1} & n_{2} \\ m & m_{1} & m_{2}\end{array}\right]=(-1)^{m+n}(2 \ell+1)\left(\begin{array}{ccc}\ell & \ell_{1} & \ell_{2} \\ -n & n_{1} & n_{2}\end{array}\right)\left(\begin{array}{ccc}\ell & \ell_{1} & \ell_{2} \\ -m & m_{1} & m_{2}\end{array}\right)$,

where $m=m_{1}+m_{2}$ and $n=n_{1}+n_{2}$. An important property of the J-square

$\left[\begin{array}{ccc}\ell & \ell_{1} & \ell_{2} \\ 0 & 0 & 0 \\ m & m_{1} & m_{2}\end{array}\right]$

is that it is zero if $\ell+\ell_{1}+\ell_{2}$ is odd.

\section{A4 Derivatives and powers of $h(q, \chi)$}

We assume that $h(q, \chi)$ is given by

$h(q, \chi)=h_{0}(q)+h_{2}(q) P_{2}(\cos \chi)+h_{4}(q) P_{4}(\cos \chi)$,

where $h_{2} / q$ is about $2 \times 10^{-3}$ for realistic earth models, both $h_{0} / q$ and $h_{4} / q$ are of the order of $\left(h_{2} / q\right)^{2}$,

$P_{2}(\cos \chi)=\mathcal{D}_{2}^{0}(\chi)=\frac{1}{2}\left(3 \cos ^{2} \chi-1\right)$

and

$P_{4}(\cos \chi)=\mathcal{D}_{4}^{0}(\chi)=\frac{1}{8}\left(35 \cos ^{4} \chi-30 \cos ^{2} \chi+3\right)$.

We need the following derivatives of $h$ :

$$
\begin{aligned}
& \frac{\partial h}{\partial \chi}=-h_{2} P_{2}^{1}-h_{4} P_{4}^{1} \\
&=-h_{2} 3 \sin \chi \mathcal{D}_{1}^{0}-h_{4} \sin \chi\left(7 \mathcal{D}_{3}^{0}+3 \mathcal{D}_{1}^{0}\right), \\
& \frac{\cos \chi}{\sin \chi} \frac{\partial h}{\partial \chi}=-h_{2}\left(2 \mathcal{D}_{2}^{0}+1\right)-h_{4}\left(4 \mathcal{D}_{4}^{0}+5 \mathcal{D}_{2}^{0}+1\right), \\
& \frac{\partial^{2} h}{\partial \chi^{2}}=-h_{2}\left(4 \mathcal{D}_{2}^{0}-1\right)-h_{4}\left(16 \mathcal{D}_{4}^{0}-5 \mathcal{D}_{2}^{0}-1\right), \\
& \frac{\partial^{2} h}{\partial \chi^{2}}+\frac{\cos \chi}{\sin \chi} \frac{\partial h}{\partial \chi}=-6 h_{2} \mathcal{D}_{2}^{0}-20 h_{4} \mathcal{D}_{4}^{0}, \\
& \frac{\partial^{3} h}{\partial \chi^{3}}=h_{2} 4 P_{2}^{1}+h_{4}\left(16 P_{4}^{1}-5 P_{2}^{1}\right) \\
&=h_{2} 12 \sin \chi \mathcal{D}_{1}^{0}+h_{4} \sin \chi\left(112 \mathcal{D}_{3}^{0}+33 \mathcal{D}_{1}^{0}\right), \\
& \frac{\partial^{4} h}{\partial \chi^{4}}=h_{2}\left(16 \mathcal{D}_{2}^{0}-4\right)+h_{4}\left(256 \mathcal{D}_{4}^{0}-100 \mathcal{D}_{2}^{0}-11\right) .
\end{aligned}
$$

In a second-order theory, we also meet expressions involving the square of $h$, the square of its derivatives, or the product of $h$ and its derivatives. Here is a list of formulae that are useful in Sections 4 and 7 :

$h^{2}=h_{2}^{2}\left(\frac{18}{35} \mathcal{D}_{4}^{0}+\frac{2}{7} \mathcal{D}_{2}^{0}+\frac{1}{5}\right)$

$\left(\frac{\partial h}{\partial \chi}\right)^{2}=h_{2}^{2}\left(-\frac{72}{35} \mathcal{D}_{4}^{0}+\frac{2}{7} \mathcal{D}_{2}^{0}+\frac{6}{5}\right)$,

$h \frac{\partial^{2} h}{\partial \chi^{2}}=h_{2}^{2}\left(-\frac{72}{35} \mathcal{D}_{4}^{0}-\frac{1}{7} \mathcal{D}_{2}^{0}-\frac{4}{5}\right)$

$\left(\frac{\partial^{2} h}{\partial \chi^{2}}\right)^{2}=h_{2}^{2}\left(\frac{288}{35} \mathcal{D}_{4}^{0}-\frac{24}{7} \mathcal{D}_{2}^{0}+\frac{21}{5}\right)$, 


$$
\begin{aligned}
& \frac{\partial h}{\partial \chi} \frac{\partial^{3} h}{\partial \chi^{3}}=h_{2}^{2}\left(\frac{288}{35} \mathcal{D}_{4}^{0}-\frac{8}{7} \mathcal{D}_{2}^{0}-\frac{24}{5}\right), \\
& h \frac{\cos \chi}{\sin \chi} \frac{\partial h}{\partial \chi}=h_{2}^{2}\left(-\frac{36}{35} \mathcal{D}_{4}^{0}-\frac{11}{7} \mathcal{D}_{2}^{0}-\frac{2}{5}\right), \\
& \frac{\cos \chi}{\sin \chi} \frac{\partial h}{\partial \chi} \frac{\partial^{2} h}{\partial \chi^{2}}=h_{2}^{2}\left(\frac{144}{35} \mathcal{D}_{4}^{0}+\frac{30}{7} \mathcal{D}_{2}^{0}+\frac{3}{5}\right), \\
& \frac{1}{\sin ^{2} \chi}\left(\frac{\partial h}{\partial \chi}\right)^{2}=h_{2}^{2}\left(6 \mathcal{D}_{2}^{0}+3\right)
\end{aligned}
$$

\section{A5 Useful formulae}

We now collect formulae that are used repeatedly in deriving the scalar equations of motion (Section 7). In the following, $f$ denotes a function of $q, \chi$, and $v$ that is expanded on the basis of the spherical harmonics:

$f(q, \chi, v)=\sum_{\ell=0}^{\infty} \sum_{m=-\ell}^{\ell} f_{\ell}^{m}(q) \mathcal{D}_{\ell}^{m}(\chi, v)$.

Also, $f_{1}$ and $f_{2}$ designate two functions of $q, \chi$, and $v$ expandable on the basis of the spherical harmonics. Using the product of two spherical harmonics and the formulae provided in A4, and knowing that $f^{m}{ }_{\ell}$ and $\mathcal{D}_{\ell}^{m}$ vanish if $|m|>\ell$, it can be shown that

$$
\begin{aligned}
& h f=\sum_{\ell=0}^{\infty} \sum_{m=-\ell}^{\ell} h f_{\ell}^{m} \mathcal{D}_{\ell}^{m} \\
&=\sum_{\ell=0}^{\infty} \sum_{m=-\ell}^{\ell}\left\{h_{0} f_{\ell}^{m}+h_{2} \sum_{\ell^{\prime}=|\ell-2|}^{\ell+2}\left[\begin{array}{ccc}
\ell & 2 & \ell^{\prime} \\
0 & 0 & 0 \\
m & 0 & m
\end{array}\right]\left\{\begin{array}{c}
f_{\ell^{\prime}}^{m} \\
0
\end{array}\right\}+h_{4} \sum_{\ell^{\prime}=|\ell-4|}^{\ell+4}\left[\begin{array}{ccc}
\ell & 4 & \ell^{\prime} \\
0 & 0 & 0 \\
m & 0 & m
\end{array}\right]\left\{\begin{array}{c}
f_{\ell^{\prime}}^{m} \\
0
\end{array}\right\}\right\} \mathcal{D}_{\ell}^{m}, \\
& \frac{\partial h}{\partial \chi} \frac{\partial f_{1}}{\partial \chi}+\frac{1}{\sin \chi} \frac{\partial h}{\partial \chi} \frac{\partial f_{2}}{\partial v}=\sum_{\ell=0}^{\infty} \sum_{m=-\ell}^{\ell}\left\{h_{2} 2 \sqrt{3} \sum_{\ell^{\prime}=|\ell-2|}^{\ell+2} L_{0}^{\ell^{\prime}}\left[\begin{array}{ccc}
\ell & 2 & \ell^{\prime} \\
0 & 1 & -1 \\
m & 0 & m
\end{array}\right]\left\{\begin{array}{c}
-f_{1}^{m} \\
i f_{2 \ell^{\prime}}^{m}
\end{array}\right\}\right. \\
&\left.+h_{4} 2 \sqrt{10} \sum_{\ell^{\prime}=|\ell-4|}^{\ell+4} L_{0}^{\ell^{\prime}}\left[\begin{array}{ccc}
\ell & 4 & \ell^{\prime} \\
0 & 1 & -1 \\
m & 0 & m
\end{array}\right]\left\{\begin{array}{c}
-f_{1 \ell^{\prime}}^{m} \\
i f_{2 \ell^{\prime}}^{m}
\end{array}\right\}\right\} \mathcal{D}_{\ell}^{m},
\end{aligned}
$$

where

$L_{0}^{\ell}=\sqrt{\frac{\ell(\ell+1)}{2}}$

In the sums over $\ell^{\prime}$, arrays with two lines in curly brackets such as

$$
\left\{\begin{array}{c}
f_{\ell^{\prime}}^{m} \\
0
\end{array}\right\}
$$

mean that the upper line must be chosen if $\left|\ell-\ell^{\prime}\right|$ is even or zero and the lower line must be chosen if $\left|\ell-\ell^{\prime}\right|$ is odd.

We also have

$$
\begin{aligned}
& \frac{\partial \mathcal{D}_{\ell}^{m}}{\partial \nu}=-i 2 L_{0}^{\ell}\left[\begin{array}{ccc}
\ell & 1 & \ell \\
0 & 1 & -1 \\
m & 0 & m
\end{array}\right] \mathcal{D}_{\ell}^{m}=i 2 L_{0}^{\ell}\left[\begin{array}{ccc}
\ell & 1 & \ell \\
-1 & -1 & 0 \\
m & 0 & m
\end{array}\right] \mathcal{D}_{\ell}^{m}=-i \ell(\ell+1)\left[\begin{array}{ccc}
\ell & 1 & \ell \\
-1 & 0 & -1 \\
m & 0 & m
\end{array}\right] \mathcal{D}_{\ell}^{m} \\
& \sin \chi \frac{\partial f}{\partial \chi}=\sum_{\ell=0}^{\infty} \sum_{m=-\ell}^{\ell}\left\{\sum_{\ell^{\prime}=|\ell-1|}^{\ell+1}\left[\begin{array}{ccc}
\ell & 1 & \ell^{\prime} \\
0 & 1 & -1 \\
m & 0 & m
\end{array}\right] 2 L_{0}^{\ell^{\prime}}\left\{\begin{array}{c}
0 \\
f_{\ell^{\prime}}^{m}
\end{array}\right\}\right\} \mathcal{D}_{\ell}^{m},
\end{aligned}
$$




$$
\begin{aligned}
& h \frac{\partial f_{1}}{\partial v}+h \sin \chi \frac{\partial f_{2}}{\partial \chi}=\sum_{\ell=0}^{\infty} \sum_{m=-\ell}^{\ell}\left\{h_{2} \sum_{\ell^{\prime}=|\ell-2|}^{\ell+2} \sum_{\ell^{\prime \prime}=\left|\ell^{\prime}-1\right|}^{\ell^{\prime}+1}\left[\begin{array}{ccc}
\ell & 2 & \ell^{\prime} \\
0 & 0 & 0 \\
m & 0 & m
\end{array}\right]\left[\begin{array}{ccc}
\ell^{\prime} & 1 & \ell^{\prime \prime} \\
0 & 1 & -1 \\
m & 0 & m
\end{array}\right] 2 L_{0}^{\ell^{\prime \prime}}\left\{\begin{array}{c}
-i f_{1}^{m} \ell^{\prime \prime} \\
f_{2}^{m} \ell^{\prime \prime}
\end{array}\right\}\right. \\
& \left.+h_{4} \sum_{\ell^{\prime}=|\ell-4| \ell^{\prime \prime}=\left|\ell^{\prime}-1\right|}^{\ell+4}\left[\begin{array}{ccc}
\ell & 4 & \ell^{\prime} \\
0 & 0 & 0 \\
m & 0 & m
\end{array}\right]\left[\begin{array}{ccc}
\ell^{\prime} & 1 & \ell^{\prime \prime} \\
0 & 1 & -1 \\
m & 0 & m
\end{array}\right] 2 L_{0}^{\ell^{\prime \prime}}\left\{\begin{array}{c}
-i f_{1}^{m} \ell^{\prime \prime} \\
f_{2 \ell^{\prime \prime}}^{m}
\end{array}\right\}\right\} \mathcal{D}_{\ell}^{m}, \\
& \frac{\cos \chi}{\sin \chi} \frac{\partial h}{\partial \chi} \frac{\partial f_{1}}{\partial v}+\cos \chi \frac{\partial h}{\partial \chi} \frac{\partial f_{2}}{\partial \chi} \\
& =\sum_{\ell=0}^{\infty} \sum_{m=-\ell}^{\ell}\left\{\left(h_{2}+h_{4}\right) \sum_{\ell^{\prime}=|\ell-1|}^{\ell+1}\left[\begin{array}{ccc}
\ell & 1 & \ell^{\prime} \\
0 & 1 & -1 \\
m & 0 & m
\end{array}\right] 2 L_{0}^{\ell^{\prime}}\left\{\begin{array}{c}
i f_{\ell^{\ell^{\prime}}}^{m} \\
-f_{2 \ell^{\prime}}^{m}
\end{array}\right\}\right. \\
& +\left(2 h_{2}+5 h_{4}\right) \sum_{\ell^{\prime}=|\ell-2| \ell^{\prime \prime}=\left|\ell^{\prime}-1\right|}^{\ell+2} \sum_{\ell^{\prime}+1}\left[\begin{array}{ccc}
\ell & 2 & \ell^{\prime} \\
0 & 0 & 0 \\
m & 0 & m
\end{array}\right]\left[\begin{array}{ccc}
\ell^{\prime} & 1 & \ell^{\prime \prime} \\
0 & 1 & -1 \\
m & 0 & m
\end{array}\right] 2 L_{0}^{\ell^{\prime \prime}}\left\{\begin{array}{c}
i f_{1}^{m} \ell^{\prime \prime} \\
-f_{2 \ell^{\prime \prime}}^{m}
\end{array}\right\} \\
& \left.+4 h_{4} \sum_{\ell^{\prime}=|\ell-4|}^{\ell+4} \sum_{\ell^{\prime \prime}=\left|\ell^{\prime}-1\right|}^{\ell^{\prime}+1}\left[\begin{array}{ccc}
\ell & 4 & \ell^{\prime} \\
0 & 0 & 0 \\
m & 0 & m
\end{array}\right]\left[\begin{array}{ccc}
\ell^{\prime} & 1 & \ell^{\prime \prime} \\
0 & 1 & -1 \\
m & 0 & m
\end{array}\right] 2 L_{0}^{\ell^{\prime \prime}}\left\{\begin{array}{c}
i f_{1}^{m} \\
-f_{2}^{\prime \prime} \ell^{\prime \prime}
\end{array}\right\}\right\} \mathcal{D}_{\ell}^{m} \text {. }
\end{aligned}
$$

In the double sums over both $\ell^{\prime}$ and $\ell^{\prime \prime}$, arrays with two lines in curly brackets such as

$$
\left\{\begin{array}{c}
f_{\ell^{\prime \prime}}^{m} \\
0
\end{array}\right\}
$$

mean that the upper line must be chosen if both $\left|\ell-\ell^{\prime}\right|$ and $\left|\ell^{\prime}-\ell^{\prime \prime}\right|$ are even, odd, or zero, and the lower line must be chosen if $\left|\ell-\ell^{\prime}\right|$ is even or zero and $\left|\ell^{\prime}-\ell^{\prime \prime}\right|$ is odd, or if $\left|\ell-\ell^{\prime}\right|$ is odd and $\left|\ell^{\prime}-\ell^{\prime \prime}\right|$ is even or zero.

Other useful formulae are

$$
\begin{aligned}
& \sin \chi \frac{\partial h}{\partial \chi} f=\sum_{\ell=0}^{\infty} \sum_{m=-\ell}^{\ell}\left\{\left(-\frac{6}{5} h_{2}\right) \sum_{\ell^{\prime}=|\ell-1|}^{\ell+1}\left[\begin{array}{ccc}
\ell & 1 & \ell^{\prime} \\
0 & 0 & 0 \\
m & 0 & m
\end{array}\right]\left\{\begin{array}{c}
0 \\
f_{\ell^{\prime}}^{m}
\end{array}\right\}+\left(\frac{6}{5} h_{2}-\frac{20}{9} h_{4}\right) \sum_{\ell^{\prime}=|\ell-3|}^{\ell+3}\left[\begin{array}{ccc}
\ell & 3 & \ell^{\prime} \\
0 & 0 & 0 \\
m & 0 & m
\end{array}\right]\left\{\begin{array}{c}
0 \\
f_{\ell^{\prime}}^{m}
\end{array}\right\}\right. \\
& \left.+\frac{20}{9} h_{4} \sum_{\ell^{\prime}=|\ell-5|}^{\ell+5}\left[\begin{array}{ccc}
\ell & 5 & \ell^{\prime} \\
0 & 0 & 0 \\
m & 0 & m
\end{array}\right]\left\{\begin{array}{c}
0 \\
f_{\ell^{\prime}}^{m}
\end{array}\right\}\right\} \mathcal{D}_{\ell}^{m} \\
& -2 f \cos \chi-\sin \chi \frac{\partial f}{\partial \chi}=-\sum_{\ell=0}^{\infty} \sum_{m=-\ell}^{\ell} L_{0}^{\ell}\left\{\sum_{\ell^{\prime}=|\ell-1|}^{\ell+1}\left[\begin{array}{ccc}
\ell & 1 & \ell^{\prime} \\
-1 & -1 & 0 \\
m & 0 & m
\end{array}\right]\left\{\begin{array}{c}
0 \\
2 f_{\ell^{\prime}}^{m}
\end{array}\right\}\right\} \mathcal{D}_{\ell}^{m}, \\
& h\left[-2 f \cos \chi-\sin \chi \frac{\partial f}{\partial \chi}\right]= \\
& \sum_{\ell=0}^{\infty} \sum_{m=-\ell}^{\ell}\left\{\left[-h_{2} \sum_{\ell^{\prime}=|\ell-2|}^{\ell+2} \sum_{\ell^{\prime \prime}=\left|\ell^{\prime}-1\right|}^{\ell^{\prime}+1} L_{0}^{\ell^{\prime}}\left[\begin{array}{ccc}
\ell & 2 & \ell^{\prime} \\
0 & 0 & 0 \\
m & 0 & m
\end{array}\right]\left[\begin{array}{ccc}
\ell^{\prime} & 1 & \ell^{\prime \prime} \\
-1 & -1 & 0 \\
m & 0 & m
\end{array}\right]\right.\right. \\
& \left.\left.-h_{4} \sum_{\ell^{\prime}=|\ell-4|}^{\ell+4} \sum_{\ell^{\prime \prime}=\left|\ell^{\prime}-1\right|}^{\ell^{\prime}+1} L_{0}^{\ell^{\prime}}\left[\begin{array}{ccc}
\ell & 4 & \ell^{\prime} \\
0 & 0 & 0 \\
m & 0 & m
\end{array}\right]\left[\begin{array}{ccc}
\ell^{\prime} & 1 & \ell^{\prime \prime} \\
-1 & -1 & 0 \\
m & 0 & m
\end{array}\right]\right]\left\{\begin{array}{c}
0 \\
2 f_{\ell^{\prime}}^{m}
\end{array}\right\}\right\} \mathcal{D}_{\ell}^{m} \text {, }
\end{aligned}
$$




$$
\begin{aligned}
& \sum_{\ell=0}^{\infty} \sum_{m=-\ell}^{\ell}\left[-\cos \chi \frac{\partial^{2} \mathcal{D}_{\ell}^{m}}{\partial \chi^{2}}+\left(\sin \chi-\frac{\cos ^{2} \chi}{\sin \chi}\right) \frac{\partial \mathcal{D}_{\ell}^{m}}{\partial \chi}-\frac{\cos \chi}{\sin ^{2} \chi} \frac{\partial^{2} \mathcal{D}_{\ell}^{m}}{\partial \nu^{2}}\right] f_{1 \ell}^{m}+\frac{\partial \mathcal{D}_{\ell}^{m}}{\partial v} f_{2 \ell}^{m} \\
& \quad=\sum_{\ell=0}^{\infty} \sum_{m=-\ell}^{\ell} L_{0}^{\ell}\left\{\sum_{\ell^{\prime}=|\ell-1|}^{\ell+1}\left[\begin{array}{ccc}
\ell & 1 & \ell^{\prime} \\
-1 & 0 & -1 \\
m & 0 & m
\end{array}\right] \sqrt{2 \ell^{\prime}\left(\ell^{\prime}+1\right)}\left\{\begin{array}{c}
-i f_{2 \ell^{\prime}}^{m} \\
f_{1 \ell^{\prime}}^{m}
\end{array}\right\}\right\} \mathcal{D}_{\ell}^{m}, \\
& \sum_{\ell=0}^{\infty} \sum_{m=-\ell}^{\ell} h\left\{\left[-\cos \chi \frac{\partial^{2} \mathcal{D}_{\ell}^{m}}{\partial \chi^{2}}+\left(\sin \chi-\frac{\cos ^{2} \chi}{\sin \chi}\right) \frac{\partial \mathcal{D}_{\ell}^{m}}{\partial \chi}-\frac{\cos \chi}{\sin ^{2} \chi} \frac{\partial^{2} \mathcal{D}_{\ell}^{m}}{\partial \nu^{2}}\right] f_{1 \ell}^{m}+\frac{\partial \mathcal{D}_{\ell}^{m}}{\partial v} f_{2 \ell}^{m}\right\} \\
& =\sum_{\ell=0}^{\infty} \sum_{m=-\ell}^{\ell}\left\{\sum_{\ell^{\prime}=|\ell-2| \ell^{\prime \prime}=\left|\ell^{\prime}-1\right|}^{\ell+2} \sum_{\ell^{\prime}+1}^{\ell}\left[\begin{array}{ccc}
\ell & 2 & \ell^{\prime} \\
0 & 0 & 0 \\
m & 0 & m
\end{array}\right]\left[\begin{array}{ccc}
\ell^{\prime} & 1 & \ell^{\prime \prime} \\
-1 & 0 & -1 \\
m & 0 & m
\end{array}\right] h_{2} L_{0}^{\ell^{\prime}} \sqrt{2 \ell^{\prime \prime}\left(\ell^{\prime \prime}+1\right)}\left\{\begin{array}{c}
-i f_{2}^{m} m \\
f_{1 \ell^{\prime \prime}}^{m}
\end{array}\right\}\right\} \mathcal{D}_{\ell}^{m},
\end{aligned}
$$

$\frac{\cos \chi}{\sin \chi}\left(\frac{\partial h}{\partial \chi}\right)^{2} \frac{\partial f}{\partial \chi}=$

$$
\begin{aligned}
& \sum_{\ell=0}^{\infty} \sum_{m=-\ell}^{\ell} h_{2}^{2} 2 \sqrt{3}\left\{\sum_{\ell^{\prime}=|\ell-2|}^{\ell+2} L_{0}^{\ell^{\prime}}\left[\begin{array}{ccc}
\ell & 2 & \ell^{\prime} \\
0 & 1 & -1 \\
m & 0 & m
\end{array}\right]\left\{\begin{array}{c}
f_{\ell^{\prime}}^{m} \\
0
\end{array}\right\}\right. \\
& \left.+\sum_{\ell^{\prime}=|\ell-2| \ell^{\prime \prime}=\left|\ell^{\prime}-2\right|}^{\ell+2} L_{0}^{\ell^{\prime \prime}}\left[\begin{array}{ccc}
\ell & 2 & \ell^{\prime} \\
0 & 0 & 0 \\
m & 0 & m
\end{array}\right]\left[\begin{array}{ccc}
\ell^{\prime} & 2 & \ell^{\prime \prime} \\
0 & 1 & -1 \\
m & 0 & m
\end{array}\right]\left\{\begin{array}{c}
f_{\ell^{\prime \prime}}^{m} \\
0
\end{array}\right\}\right\} \mathcal{D}_{\ell}^{m},
\end{aligned}
$$

$\frac{1}{\sin ^{2} \chi}\left(\frac{\partial h}{\partial \chi}\right)^{2} \frac{\partial^{2} f}{\partial v^{2}}=$

$$
\sum_{\ell=0}^{\infty} \sum_{m=-\ell}^{\ell} h_{2}^{2}\left\{-3 m^{2} f_{\ell}^{m}-\sum_{\ell^{\prime}=|\ell-2|}^{\ell+2} 6 m^{2}\left[\begin{array}{ccc}
\ell & 2 & \ell^{\prime} \\
0 & 0 & 0 \\
m & 0 & m
\end{array}\right]\left\{\begin{array}{c}
f_{\ell^{\prime}}^{m} \\
0
\end{array}\right\}\right\} \mathcal{D}_{\ell}^{m},
$$

and

$$
\begin{aligned}
& \frac{1}{\sin \chi}\left(\frac{\partial h}{\partial \chi}\right)^{2} \frac{\partial^{2} f}{\partial \chi \partial \nu}= \\
& \sum_{\ell=0}^{\infty} \sum_{m=-\ell}^{\ell} \sum_{\ell^{\prime}=|\ell-2| \ell^{\prime \prime}=\left|\ell^{\prime}-2\right|}^{\ell+2} \sum^{\ell^{\prime}+2}\left\{12 L_{0}^{\ell} L_{0}^{\ell^{\prime \prime}} h_{2}^{2}\left[\begin{array}{ccc}
\ell & 2 & \ell^{\prime} \\
0 & 1 & -1 \\
m & 0 & m
\end{array}\right]\left[\begin{array}{ccc}
\ell^{\prime} & 2 & \ell^{\prime \prime} \\
0 & 1 & -1 \\
m & 0 & m
\end{array}\right]\left\{\begin{array}{c}
0 \\
-i f_{\ell^{\prime \prime}}^{m}
\end{array}\right\}\right\} \mathcal{D}_{\ell}^{m} .
\end{aligned}
$$

